\title{
Theory of classical metastability in open quantum systems
}

\author{
Katarzyna Macieszczak $\odot,{ }^{1}$ Dominic C. Rose $\odot,{ }^{2,3}$ Igor Lesanovsky, ${ }^{4,2,3}$ and Juan P. Garrahan ${ }^{2,3}$ \\ ${ }^{1}$ TCM Group, Cavendish Laboratory, University of Cambridge, J. J. Thomson Avenue, Cambridge CB3 OHE, United Kingdom \\ ${ }^{2}$ School of Physics and Astronomy, University of Nottingham, University Park, Nottingham NG7 2RD, United Kingdom \\ ${ }^{3}$ Centre for the Mathematics and Theoretical Physics of Quantum Non-Equilibrium Systems, University of Nottingham, \\ University Park, Nottingham NG7 2RD, United Kingdom \\ ${ }^{4}$ Institut für Theoretische Physik, Universität Tübingen, Auf der Morgenstelle 14, 72076 Tübingen, Germany
}

(Received 27 May 2020; accepted 18 June 2021; published 12 July 2021)

\begin{abstract}
We present a general theory of classical metastability in open quantum systems. Metastability is a consequence of a large separation in timescales in the dynamics, leading to the existence of a regime when states of the system appear stationary, before eventual relaxation toward a true stationary state at much larger times. In this work, we focus on the emergence of classical metastability, i.e., when metastable states of an open quantum system with separation of timescales can be approximated as probabilistic mixtures of a finite number of states. We find that a number of classical features follow from this approximation, for the manifold of metastable states, long-time dynamics between them, and symmetries of the dynamics. Namely, those states are approximately disjoint and thus play the role of metastable phases, the relaxation toward the stationary state is approximated by a classical stochastic dynamics between them, and weak symmetries correspond to their permutations. Importantly, the classical dynamics is observed not only on average but also at the level of individual quantum trajectories: We show that time coarse-grained continuous measurement records can be viewed as noisy classical trajectories, while their statistics can be approximated by that of the classical dynamics. Among others, this explains how first-order dynamical phase transitions arise from metastability. Finally, to verify the presence of classical metastability in a given open quantum system, we develop an efficient numerical approach that delivers the set of metastable phases together with the effective classical dynamics. Since the proximity to a first-order dissipative phase transition manifests as metastability, the theory and tools introduced in this work can be used to investigate such transitions-which occur in the large size limit—-through the metastable behavior of many-body systems of moderate sizes accessible to numerics.
\end{abstract}

DOI: 10.1103/PhysRevResearch.3.033047

\section{INTRODUCTION}

With continuing advances in the control of experimental platforms used as quantum simulators, such as ultracold atomic gases, Rydberg atoms, and circuit quantumelectrodynamics [1-7], a broad range of nonequilibrium phenomena of open many-body quantum systems has been observed recently. Theoretical studies have progressed via the combination of methods from atomic physics, quantum optics, and condensed matter, giving rise to a range of techniques, including quantum jump Monte Carlo (QJMC) [8-12] simulations via tensor network [13], and field theoretical approaches [14-16].

Often the focus of studies on nonequilibrium open manybody quantum systems is a phase diagram of the stationary state, and the related question of the structure of dissipative phase transitions occurring in the thermodynamic limit of

Published by the American Physical Society under the terms of the Creative Commons Attribution 4.0 International license. Further distribution of this work must maintain attribution to the author(s) and the published article's title, journal citation, and DOI. infinite system size. This includes whether such systems can exhibit bistability (or multistability) of the stationary state, a topic covered both theoretically [17-21] and experimentally $[22,23]$, and which order parameters are relevant for distinguishing the coexisting phases. Mean-field results often suggest multiple stationary states in the thermodynamic limit [16,24,25]; however, more sophisticated (albeit still approximate) techniques such as variational approaches [26-28], perturbative expansions in lattice connectivity [25,29], infinite tensor network simulations [13], or a field-theoretical analysis [16] can still indicate a unique stationary state.

While it is unusual to see phase transitions at finite system sizes [30-33], first-order phase transitions in stationary states manifest at large enough finite system sizes [34] through the occurrence of metastability, i.e., distinct timescales in the evolution of the system statistics: classically, in the probability distribution over configuration space [35-39]; quantum mechanically, in the density matrix [40,41]. The statistics of such systems at long times can be understood in terms of metastable phases which generally correspond to the phases on either side of the transition being distinct from the unique stationary state for a given set of parameters. Therefore, already at a finite system size the structure of a possible first-order dissipative phase transition can be fully determined 
by investigating metastable states of the system [41,42], which is of particular importance for many-body open quantum systems, where exact methods are often limited to numerical simulations of finite systems of modest size.

Metastability can also emerge in complex relaxation toward a unique stationary state, even without a phase transition present in the thermodynamic limit. This is the case in classical kinetically constrained models [43-48] and spin glasses [49] and recent open quantum generalizations of the models in Refs. [50-52] and Ref. [53], respectively. Here, the study of metastability can unfold the long-time dynamics responsible for the complex relaxation to the stationary state [54], with metastable phases corresponding to dynamical rather than static phases.

For classical systems with Markovian dynamics [35-39] and open quantum systems $[40,56]$ described by the master equation formalism [57,58], metastability necessarily requires a large separation in the spectrum of the master operator governing the system evolution. This separation leads to metastable states residing in a space of a reduced dimension given by the slow eigenmodes of the master operator, and long-time dynamics taking place within that space. Since the slow modes themselves do not represent system states, a general structure of the metastable manifold (MM) is not known, but conjectured to feature disjoint phases, decoherence free subspaces and noiseless subsystems, while the long-time dynamics is expected to be analogous to perturbative dynamics on such states [40].

In this work, we comprehensively prove this conjecture for classical metastability in open quantum systems. We define classical metastability as the case, where metastable states can be approximated as probability distributions over a set of $m$ states, where $m-1$ is the number of slow eigenmodes in the dynamics. We then show that this definition is equivalent to a simple geometric criterion, which can be verified using the exact diagonalization of the master operator. Crucially, the corresponding corrections play the role of a figure of merit in emergent classical properties of the manifold of metastable states and its long-time dynamics. Namely, we show that, for classical metastability, $m$ states can be considered as distinct metastable phases, as they are approximately disjoint and orthogonal to one another, while their basins of attraction form the set of $m$ order parameters to distinguish them. Furthermore, we find that the long-time dynamics of the system can be approximated as a classical stochastic dynamics between the metastable phases. This holds in the average system dynamics as well as in individual quantum trajectories [12,59], as obtained via individual runs of an experimental system or from QJMC simulations, where classical trajectories arise via coarse graining of these in time. The classical dynamics between the metastable phases is then responsible for the occurrence of intermittence [24,41,51] or dynamical heterogeneity $[50,52]$ in quantum trajectories, leading to multimodal statistics of continuous measurements and system proximity to a first-order dynamical phase transition [60]. Therefore, classical metastability is a phenomenon occurring not only on average, but in dynamics of individual quantum trajectories. All these results are also discussed in the presence of further hierarchy of relaxation timescales. Finally, while our approach does not rely on the presence of any weak symmetries of the dynamics [61-63] (cf. Ref. [42]), we also show that the set of metastable phases is approximately invariant under any present symmetries. Thus, weak symmetries lead to approximate cycles of metastable phases and permutation symmetries of the classical long-time dynamics, and, as such, we find that any nontrivial continuous symmetries of slow eigenmodes of the dynamics preclude classical metastability.

To verify the classicality of metastability present in a general open quantum system and uncover the set of metastable phases together with the effective structure of long-time dynamics, we develop an efficient numerical technique, which can be further simplified when a dynamical symmetry is present. Our approach relies on the ability to diagonalize the system master operator, which is usually possible only for moderate system sizes, while metastability may become prominent only for large system sizes. To mitigate this potential issue, we show that for classical metastability accompanied by intermittence or dynamical heterogeneity in quantum trajectories, metastable phases can be extracted from quantum trajectories through the use of large-deviation methods, such as the "thermodynamics of trajectories" [24,51,60,64,65]. Therefore, there is potential to study classical metastability using QJMC simulations, which are generally feasible at quadratically larger system sizes than exact diagonalization of the generator.

This paper is organized as follows. In Sec. II, we review the results of Ref. [40]. In Sec. III, we introduce the general approach to classical metastability in open quantum systems. We then discuss the resulting classical structure of the MM in Sec. IV. The effectively classical system dynamics emerging at large times is discussed in Sec. V. We refine these general results considering symmetries of the system dynamics in Sec. VI. Finally, we introduce numerical approaches to unfold the structure of classical metastability in Sec. VII. Details and proofs of our results are presented in the Supplemental Material [55]. Our results are illustrated in Figs. 1-5 with a system in proximity to an effectively classical dissipative phase transition occurring at finite size. We discuss classical metastability arising for such systems in Sec. A of the Supplemental Material [55]. The application of the general methods introduced in this paper to a many-body system beyond this class is given in the accompanying paper [54] which studies in detail the metastability of the open quantum East glass model [50].

\section{METASTABILITY IN OPEN QUANTUM SYSTEMS}

We begin by reviewing the spectral theory of metastability of Ref. [40]. We then introduce a quantitative description of those results by considering the corrections to the stationarity during the metastable regime. In the next section, we build on this to understand when metastability in open quantum systems becomes classical, which is the main focus of this work.

\section{A. Dynamics of open quantum systems}

We consider a finitely dimensional open quantum system with ts average state at time $t$ described by a density matrix $\rho(t)$ evolving according to a master equation as 
$d \rho(t) / d t=\mathcal{L}[\rho(t)]$, where the master operator $[57,58]$

$$
\mathcal{L}(\rho)=-i[H, \rho]+\sum_{j}\left(J_{j} \rho J_{j}^{\dagger}-\frac{1}{2}\left\{J_{j}^{\dagger} J_{j}, \rho\right\}\right) .
$$

Here, $H$ is the system Hamiltonian, while the jump operators $J_{j}$ provide coupling of the system to the surrounding environment (in this work we explicitly denote any time dependence; in particular, the master equation is time-independent). If the interactions between the system and the environment are associated to emissions of an energy quanta, then the action of jump operators can be detected through continuous measurements [59], e.g., counting of photons emitted by atoms coupled to the vacuum electromagnetic field [24,50-52]. Equation (1) is a general dynamics of a time-homogeneous Markovian open quantum system [57,58], which arises for systems interacting weakly with an effectively memoryless environment [59].

Since the master operator $\mathcal{L}$ acts linearly on $\rho(t)$, the evolution can be understood in terms of its eigenmatrices $R_{k}$ and their corresponding eigenvalues $\lambda_{k}=\lambda_{k}^{R}+i \lambda_{k}^{I}$ [66]. The real parts of these eigenvalues are not greater than $0, \lambda_{k}^{R} \leqslant 0$, as the dynamics in Eq. (1) is (completely) positive and trace preserving; we order the eigenvalues by decreasing real part $\lambda_{k}^{R}$. In particular, zero eigenvalues correspond to stationary states [61,67]. In this work, we assume a generic case of a unique stationary state $R_{1}=\rho_{\mathrm{ss}}$ [30-33]. The system state at time $t$ can be then decomposed as

$$
\rho(t)=e^{t \mathcal{L}}[\rho(0)]=\rho_{\mathrm{ss}}+\sum_{k \geqslant 2} c_{k} e^{t \lambda_{k}} R_{k},
$$

where the coefficients $c_{k} \equiv \operatorname{Tr}\left[L_{k} \rho(0)\right]$ are bounded by the eigenvalues of $L_{k}$, with $L_{k}$ being eigenmatrices of $\mathcal{L}^{\dagger}$ normalized such that $\operatorname{Tr}\left(L_{k} R_{l}\right)=\delta_{k l}$ (there is a freedom of choice to normalize by scaling either $R_{k}$ or $L_{k}$ ). The values of these coefficients for a given physical state are closely tied, such that the corresponding linear combination of $R_{k}$ results in a positive matrix. We refer to $L_{k}$ and $R_{k}$ as left and right eigenmatrices (eigenmodes), respectively. Note that the trace preservation of the dynamics implies that $L_{1}=\mathbb{1}$, and thus beyond $\rho_{\mathrm{ss}}$ other right eigenmatrices do not correspond to quantum states, $\operatorname{Tr}\left(R_{k}\right)=\operatorname{Tr}\left(L_{1} R_{k}\right)=0$ for $k \geqslant 2$. The timescale $\tau$ of the final relaxation to $\rho_{\mathrm{ss}}$ from Eq. (2) can be seen to depend on the gap in the spectrum, $\tau \geqslant-1 / \lambda_{2}^{R}$.

\section{B. Spectral theory of metastability}

Metastability corresponds to a large separation in the real part of the spectrum [40], $\lambda_{m}^{R} / \lambda_{m+1}^{R} \ll 1$, which denotes the ratio of eigenvalues being of a lower order than 1 ; see Fig. 1(a). Time $t$ after the initial relaxation correspond to the terms beyond the $m$-th in the sum in Eq. (2) being negligible, $e^{t \lambda_{k}} \approx 0$ for $k \geqslant m+1$, and the reduced expansion

$$
\rho(t)=\rho_{\mathrm{ss}}+\sum_{k=2}^{m} c_{k} e^{t \lambda_{k}} R_{k}+\cdots,
$$

where ... stands for negligible corrections [cf. Eq. (2)].

When the separation in the spectrum is big enough, it is possible to further consider times when decay of the remaining terms can be neglected, $e^{t \lambda_{k}^{R}} \approx 1$ for $k \leqslant m$; cf. Ref. [68].
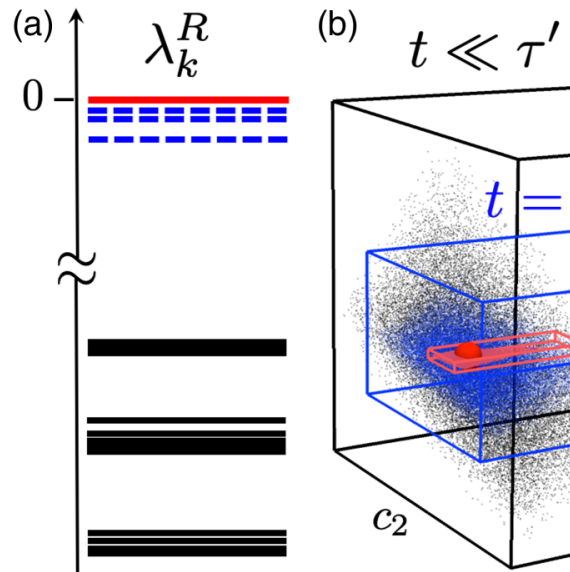

(c)

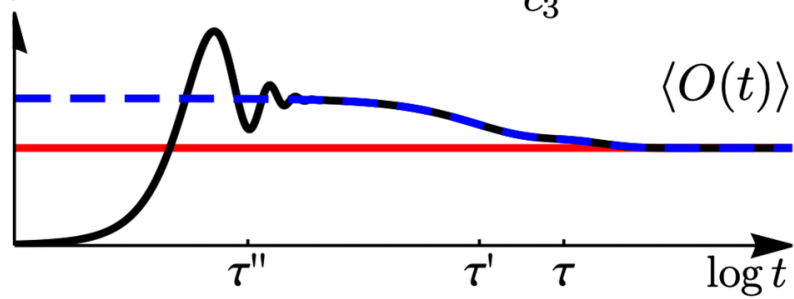

(d)

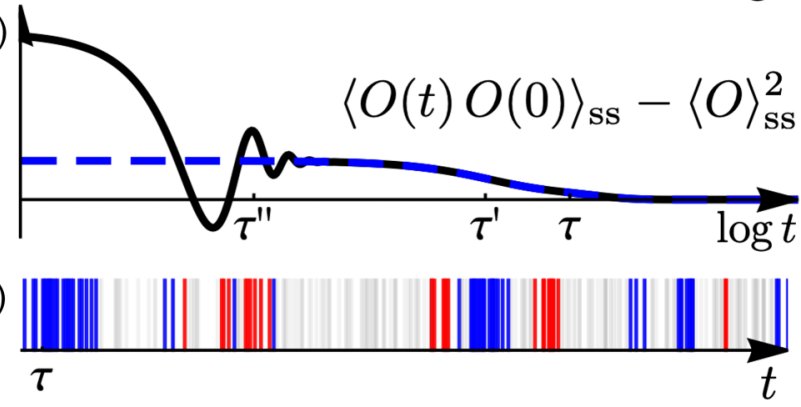

FIG. 1. Metastability in Markovian open quantum systems: (a) Metastability corresponds to a separation in the real part of master operator spectrum, between $m-1$ slow (blue dashed) and fast modes (black solid), while the stationary state corresponds to 0 eigenvalue (red solid); here $m=4$. (b) The manifold of metastable states is described by coefficients $c_{k}$ [Eq. (4)], $k=2, \ldots, m$, of decomposition between slow modes (dots for random initial pure states). The longtime dynamics takes place within that manifold, with the exponential decay of the coefficients toward the stationary state (red sphere) [Eqs. (3) and (5)]. Metastability can be observed experimentally as a plateau in the dynamics of observable averages (c) or two-point correlations (d) appearing during the metastable regime [Eqs. (8) and (9)]. Black (solid) lines show observable dynamics, blue (dashed) lines the approximation by slow modes holding after the initial relaxation, and red (solid) lines the stationary value achieved after the final relaxation. (e) Long timescales can also be observed in continuous measurement records, e.g., as intermittence in detection of quanta emitted due to jumps occurring in the system (two types shown in blue and red; gray - without associated quanta), with regimes of jump activity having a length comparable to the long-time relaxation timescale. See Sec. A1 in Ref. [55] for details on the model.

This is the metastable regime, during which the system state is approximately stationary, i.e., metastable, as captured by

$$
\rho(t)=\rho_{\mathrm{ss}}+\sum_{k=2}^{m} c_{k} R_{k}+\cdots \equiv \mathcal{P}[\rho(0)]+\cdots,
$$


where we defined $\mathcal{P}$ as the projection onto the low-lying eigenmodes of the master operator, which is trace and Hermiticity preserving [69]. From Eq. (4) the manifold of metastable states is fully characterized by the bounded coefficients $\left(c_{2}, \ldots, c_{m}\right)$ and thus it is $(m-1)$-dimensional. The $\mathrm{MM}$ is also convex, as a linear transformation of the convex set of initial states [see Fig. 1(b)].

At later times, only the slow modes contribute to the evolution [cf. Eq. (3)]. Therefore, the dynamics toward the stationary state takes place essentially inside the MM [see Fig. 1(b)],

$$
\rho(t)=e^{t \mathcal{L}_{\mathrm{MM}}} \mathcal{P}[\rho(0)]+\cdots,
$$

and is generated by [cf. Figs. 1(c) and 1(d)]

$$
\mathcal{L}_{\mathrm{MM}} \equiv \mathcal{P} \mathcal{L} \mathcal{P} .
$$

Denoting by $\tau^{\prime \prime}$ the timescale of the initial relaxation, from Eq. (3) we have $\tau^{\prime \prime} \geqslant-1 / \lambda_{m+1}^{R}$. Similarly, for $\tau^{\prime}$ being the smallest timescale of the long-time dynamics we have $\tau^{\prime} \leqslant$ $-1 / \lambda_{m}^{R}$ from Eq. (5). Then for times within the metastable regime we have $\tau^{\prime \prime} \leqslant t \ll \tau^{\prime}$ from Eq. (4) [cf. Fig. 1(b)].

Metastability can be observed in the behavior of statistical quantities such as expectation values or autocorrelations of system observables $[40,41,70]$. For a system observable, e.g., spin magnetization, we have

$$
\begin{aligned}
\langle O(t)\rangle & \equiv \operatorname{Tr}[O \rho(t)]=\operatorname{Tr}\left\{O e^{t \mathcal{L}}[\rho(0)]\right\} \\
& =\langle O\rangle_{\mathrm{ss}}+\sum_{k} b_{k} c_{k} e^{t \lambda_{k}},
\end{aligned}
$$

where we introduced decomposition of the observable into the left eigenmodes with the coefficients $b_{k} \equiv \operatorname{Tr}\left(O R_{k}\right)$ [cf. Eq. (2)], and $b_{1}=\langle O\rangle_{\mathrm{ss}}=\operatorname{Tr}\left(O \rho_{\mathrm{ss}}\right)$ is the static average. After the initial relaxation, the contribution from fast modes can be neglected [cf. Eq. (3)],

$$
\begin{aligned}
\langle O(t)\rangle & =\langle O\rangle_{\mathrm{ss}}+\sum_{k=2}^{m} b_{k} c_{k} e^{t \lambda_{k}}+\cdots \\
& =\operatorname{Tr}\left\{O e^{t \mathcal{L}_{\mathrm{MM}}} \mathcal{P}[\rho(0)]\right\}+\cdots,
\end{aligned}
$$

and the observable dynamics in Eq. (7) is accurately captured by the effective long-time dynamics in Eq. (6). Importantly, during the metastable regime, the observable average is approximately stationary [cf. Eq. (3)], before the final relaxation to $\langle O\rangle_{\mathrm{ss}}$ [see Fig. 1(c)], allowing for a direct observation of the metastability. This, however, requires preparation of an initial system state different from the stationary state, $\rho(0) \neq \rho_{\text {ss }}$, something often difficult to achieve in experimental settings. Nevertheless, for the system in the stationary state, metastability can be observed as double-step decay in the time-autocorrelation of a system observable. This is a consequence of the first measurement perturbing the stationary state, thus causing its subsequent evolution, which for times after the initial relaxation follows the effective dynamics [cf. Eq. (8)],

$$
\begin{aligned}
& \langle O(t) O(0)\rangle_{\mathrm{ss}}-\langle O\rangle_{\mathrm{ss}}^{2} \\
& \quad=\operatorname{Tr}\left[\mathcal{O} e^{t \mathcal{L}} \mathcal{O}\left(\rho_{\mathrm{ss}}\right)\right]-\langle O\rangle_{\mathrm{ss}}^{2} \\
& \quad=\operatorname{Tr}\left[\mathcal{O} e^{t \mathcal{L}_{\mathrm{MM}}} \mathcal{P} \mathcal{O}\left(\rho_{\mathrm{ss}}\right)\right]-\langle O\rangle_{\mathrm{ss}}^{2}+\cdots,
\end{aligned}
$$

where $\mathcal{O}$ denotes the superoperator representing the measurement of the observable $O$ on a system state [40,41]. The autocorrelation initially decays from the observable variance in the stationary state, $\left\langle O^{2}\right\rangle_{\mathrm{ss}}-\langle O\rangle_{\mathrm{ss}}^{2}$, to the plateau at $\operatorname{Tr}\left[\mathcal{O P O}\left(\rho_{\mathrm{ss}}\right)\right]-\langle O\rangle_{\mathrm{ss}}^{2}$ in the metastable regime, and afterwards to 0 during the final relaxation [see Fig. 1(d)].

\section{Quantitative approach}

In this work, we introduce a quantitative description of metastability. We later use this approach to prove our main results: emerging classical features of metastable manifold, long-time dynamics, and weak symmetries in the case of classical metastability.

We consider errors of the approximation of the system dynamics by the projection on the low-lying modes of the spectrum in Eq. (4) during a time regime $t^{\prime \prime} \leqslant t \leqslant t^{\prime}$,

$$
\begin{aligned}
\mathcal{C}_{\mathrm{MM}}\left(t^{\prime \prime}, t^{\prime}\right) & \equiv \sup _{\rho(0)} \sup _{t^{\prime \prime} \leqslant t \leqslant t^{\prime}}\|\rho(t)-\mathcal{P}[\rho(0)]\| \\
& =\sup _{t^{\prime \prime} \leqslant t \leqslant t^{\prime}}\left\|e^{t \mathcal{L}}-\mathcal{P}\right\|,
\end{aligned}
$$

which we refer to as the corrections to the stationarity. Here, $\|X\|=\operatorname{Tr}\left(\sqrt{X^{\dagger} X}\right)$ denotes the trace norm for an operator $X$, while for a superoperator it denotes the norm induced by the trace norm [71]. For the time regime such that

$$
\mathcal{C}_{\mathrm{MM}}\left(t^{\prime \prime}, t^{\prime}\right) \ll 1,
$$

the corrections in Eq. (4) are negligible (note that density matrices are normalized in the trace norm), which requires $t^{\prime \prime}>-1 / \lambda_{m+1}^{R}$ and $t^{\prime} \ll-1 / \lambda_{m}^{R}$ (i.e., $-\lambda_{m}^{R} t^{\prime} \ll 1$ ); see Sec. B2 in Ref. [55]. We refer to such a time regime as a metastable time regime.

We now argue that the corrections to the stationarity can be considered as the central figure of merit in the theory of metastability. Indeed, the corrections to the positivity of metastable states projected on the low-lying modes are defined by the distance to the set of density matrices,

$$
\begin{aligned}
\mathcal{C}_{+} & \equiv \sup _{\rho(0)} \inf _{\rho}\|\mathcal{P}[\rho(0)]-\rho\| \\
& =\sup _{\rho(0)}\|\mathcal{P}[\rho(0)]\|-1 \equiv\|\mathcal{P}\|-1,
\end{aligned}
$$

with $\rho$ and $\rho(0)$ being density matrices [71] (see Sec. B1 in Ref. [55]), and can be bounded by the corrections to the stationarity in Eq. (10), by considering the distance to $\rho \equiv \rho(t)$ within the metastable regime,

$$
\mathcal{C}_{+} \leqslant \inf _{t^{\prime \prime} \leqslant t \leqslant t^{\prime}}\left\|e^{t \mathcal{L}}-\mathcal{P}\right\| \equiv \tilde{\mathcal{C}}_{+}\left(t^{\prime \prime}, t^{\prime}\right) \leqslant \mathcal{C}_{\mathrm{MM}}\left(t^{\prime \prime}, t^{\prime}\right) .
$$

Furthermore, the corrections to the stationarity in Eq. (10) establish a bound not only on Eq. (12), but also on the corrections in Eqs. (2), (5), (8), and (9). In fact, beyond the metastable regime, the corrections in Eq. (5) decay exponentially, as in the leading order they can be shown to be bounded by $2 \mathcal{C}_{\mathrm{MM}}^{n}\left(t^{\prime \prime}, t^{\prime}\right)$, where $n$ is an integer such that $t / n$ belongs to the metastable regime [72]. Similarly, the corrections to observable averages and correlations in Eqs. (8) and (9) are bounded by $2 \mathcal{C}_{\mathrm{MM}}^{n}\left(t^{\prime \prime}, t^{\prime}\right)\|O\|_{\max }$ and $2 \mathcal{C}_{\mathrm{MM}}^{n}\left(t^{\prime \prime}, t^{\prime}\right)\|O\|_{\max }^{2}$, respectively, where $\|O\|_{\max }$ denotes the maximum singular value of $O$. We thus conclude that the corrections to the stationarity in Eq. (10) are a figure of merit in the theory of 
metastability. This is further confirmed by the role played by them in the errors of classical approximations for the structure of the metastable states and the long-time dynamics when classical metastability occurs, which we discuss in later sections.

We note that due to the way the condition in Eq. (11) is formulated, a choice of the metastable regime is not unique. Indeed, the corrections in Eq. (10) grow when $t^{\prime}$ increases or $t^{\prime \prime}$ decreases. In particular, extending the length $t^{\prime}-t^{\prime \prime}$ of the metastable regime $n$ times leads to the corresponding corrections the stationarity bounded in the leading order by $(n+3) \mathcal{C}_{\mathrm{MM}}\left(t^{\prime \prime}, t^{\prime}\right)$ [73]. In the rest of this work, we consider a given choice of the metastable regime for an open quantum system displaying metastability and denote $\mathcal{C}_{\mathrm{MM}}\left(t^{\prime \prime}, t^{\prime}\right)$ and $\tilde{\mathcal{C}}_{+}\left(t^{\prime \prime}, t^{\prime}\right)$ by $\mathcal{C}_{\mathrm{MM}}$ and $\tilde{\mathcal{C}}_{+}$, respectively. As a pronounced metastable regime is a hallmark of metastability phenomenon, however, some of our results rely on it being much longer than the initial relaxation time: the classical hierarchy of metastable phases discussed in Sec. IV on $t^{\prime}-t^{\prime \prime} \geqslant t^{\prime \prime}$, and the correspondence of coarse-grained quantum trajectories to classical stochastic trajectories discussed in Secs. V B 3 and VII B on $t^{\prime}-t^{\prime \prime} \geqslant n t^{\prime \prime}$ with $1 / n \ll 1$.

Finally, an analogous approach to Eq. (10) can be introduced to formally define the timescales $\tau^{\prime \prime}, \tau^{\prime}$ and $\tau$; see Sec. B3 in Ref. [55]. In particular, the relations $-1 / \lambda_{m+1}^{R} \leqslant \tau^{\prime \prime}<$ $t^{\prime \prime}, t^{\prime} \ll \tau^{\prime} \leqslant-1 / \lambda_{m}^{R}$, and $-1 / \lambda_{2}^{R} \leqslant \tau$ follow (cf. Sec. II B).

\section{Dissipative phase transitions}

When metastability is a consequence of approaching a first-order dissipative phase transition, we have by definition $\lambda_{m}^{R} / \lambda_{m+1}^{R} \rightarrow 0$ and the ratios of the timescales for the final and the initial relaxation diverge $\left(\tau^{\prime} / \tau^{\prime \prime}, \tau / \tau^{\prime \prime} \rightarrow \infty\right)$. Therefore, the ratios $t^{\prime \prime} / \tau^{\prime \prime}$ and $\tau^{\prime} / t^{\prime}$ for the metastable regime can be chosen arbitrarily large leading to all corrections arbitrarily small, $\mathcal{C}_{+}, \mathcal{C}_{\mathrm{MM}} \rightarrow$ 0; cf. Sec. B3 in Ref. [55].

\section{CLASSICAL METASTABILITY IN OPEN QUANTUM SYSTEMS}

We now introduce the notion of classical metastability, by the virtue of approximation of metastable states by probabilistic mixtures of a finite number of system states. We show that this definition can be translated into a geometric criterion on the decomposition of metastable states in projections of those states. The corresponding corrections, together with the corrections to the stationarity and the positivity, play the central role in emerging classical approximations to the structure of the metastable manifold, long-time dynamics, and weak symmetries, discussed in Secs. IV-VI. Therefore, our criterion identifies the parent feature and the figure of merit that govern the phenomenon of classical metastability in open quantum systems.

\section{A. Definition of classical metastability}

We define classical metastability to take place when any state of the system during the metastable regime $t^{\prime \prime} \leqslant t \leqslant t^{\prime}$ can be approximated as a probabilistic mixture of $m$ states

$$
\begin{aligned}
\rho_{l} \geqslant 0 \text { with } \operatorname{Tr}\left(\rho_{l}\right)=1, l & =1, \ldots, m \\
\rho(t) & =\sum_{l=1}^{m} p_{l} \rho_{l}+\cdots,
\end{aligned}
$$

where $p_{l} \geqslant 0$ with $\sum_{l=1}^{m} p_{l}=1$ represent the probabilities that depend only on an initial system state $\rho(0)$, while $\rho_{l}$, $l=1, \ldots, m$, are independent from both time and the initial state. That is, the corresponding corrections in the trace norm

$$
\mathcal{C}\left(\rho_{1}, \ldots, \rho_{m}\right) \equiv \sup _{\rho(0)} \inf _{p_{1}, . ., p_{m}} \sup _{t^{\prime \prime} \leqslant t \leqslant t^{\prime}}\left\|\rho(t)-\sum_{l=1}^{m} p_{l} \rho_{l}\right\|,
$$

fulfill

$$
\mathcal{C}\left(\rho_{1}, \ldots, \rho_{m}\right) \ll 1 .
$$

Here, the corrections depend on the choice of a metastable regime, but, for simplicity, we do not include it in the notation. We refer to $\rho_{l}$ as metastable phases (although their metastability is not assumed, but it is proven to follow together with their approximate disjointness in Sec. IV, where we also discuss their nonuniqueness). The number of phases in Eq. (14) is motivated by uniqueness of the decomposition (see also Sec. C1 of Ref. [55]) and the structure of first-order phase transitions in classical Markovian dynamics, where $m$ disjoint stationary probability distributions constitute stable phases of the system, and the system is asymptotically found in a probabilistic mixture of those phases, with probabilities depending on the initial system configuration. In later Secs. IV-V B we show that classical properties of metastable phases and long-time dynamics akin to those in proximity to a first-order transition in a classical system follow as well.

Remarkably, any metastable state in classical Markovian dynamics can be approximated by a probabilistic mixture of approximately disjoint metastable phases [35-39], whether metastability results from proximity to a first-order phase transition, or from constrained dynamics as in glassy systems. In open quantum dynamics, for the bimodal case $m=2$, it is known that any metastable state is a probabilistic mixture of two approximately disjoint metastable phases [40,41]. For higher dimensional MMs, however, the general structure is not known. Furthermore, it may be no longer classical [40], as not only disjoint phases, but also decoherence free subspaces [74-76] and noiseless subsystems [77,78] can be metastable, e.g., when perturbed away from a dissipative phase transition at a finite system size [61] (see also Supplemental Material in Ref. [40] and cf. Refs. [79-82]). Therefore, it is important to be able to verify whether a MM of an open quantum system is classical as defined in Eq. (14). In this section, we introduce such a systematic approach based on a geometric criterion equivalent to the definition in Eq. (14), and refer to it as the test of classicality.

\section{B. Test of classicality}

For a given set of $m$ candidate system states, the test of classicality enables one to verify the approximation of Eq. (14) and thus the classical metastability. Furthermore, it facilitates a check of whether a given set of $m$ initial states evolve into such metastable phases. Based on this, in Sec. VII A we introduce an efficient numerical technique delivering candidate 

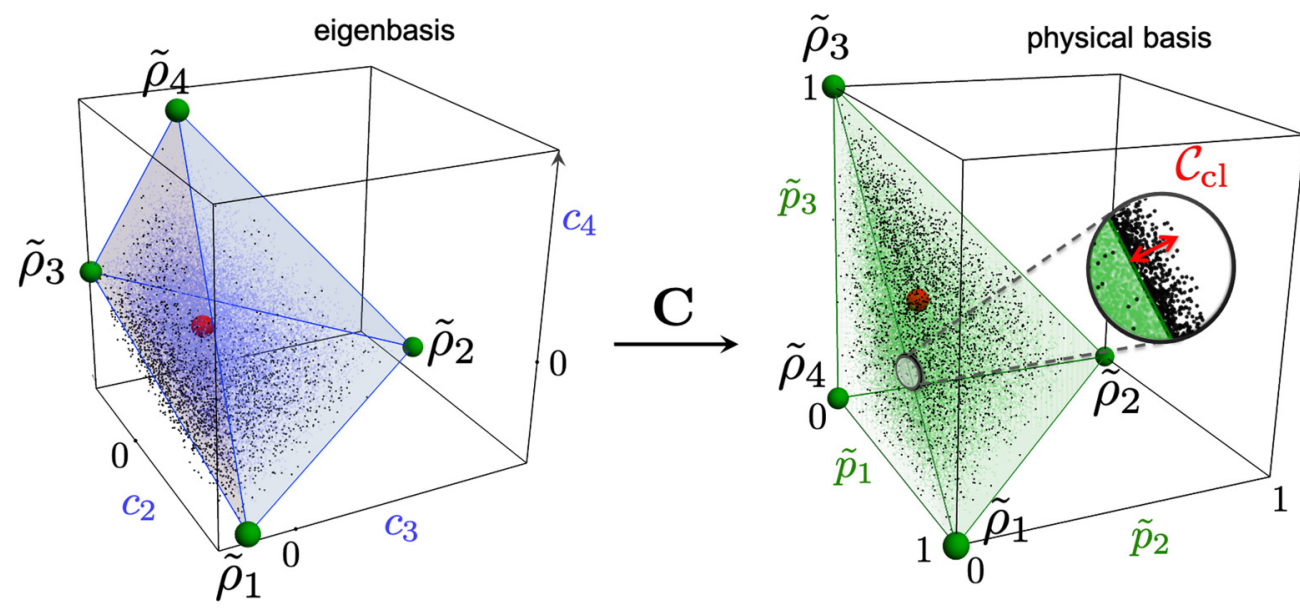

FIG. 2. Classical metastability: (left) In the space of coefficients $\left(c_{2}, c_{3}, c_{4}\right)$ [Eq. (4)], the MM features the stationary state at $(0,0,0)$ (red sphere) and is approximated by the simplex (blue lines) of $m=4$ metastable phases (green spheres at the vertices). The dots represent metastable states from randomly generated pure states found inside (blue) and outside (black) the simplex. (right) Barycentric coordinates $\left(\tilde{p}_{1}, \tilde{p}_{2}, \tilde{p}_{3}\right)$ (with $\tilde{p}_{4}=1-\sum_{l=1}^{3} \tilde{p}_{l}$ ) obtained by the transformation $\mathbf{C}$ [Eq. (18)] to the physical basis of metastable phases [Eq. (17)] yield probability distributions for states inside the simplex (green), while for states outside the simplex (black) the maximal distance $\mathcal{C}_{\mathrm{cl}}$ becomes the figure of merit for classical metastability [Eq. (21)].

states which, with the help of the test of classicality, can be postselected into metastable phases forming classical MMs.

We first note that the definition of classicality in Eq. (14) leads to the MM in the space of coefficients being approximated by a simplex (see Fig. 2). When the MM is classical, the coefficients $c_{k}$ of a general initial state $\rho(0)$ can be approximated from Eq. (4) as $\sum_{l=1}^{m} p_{l} c_{k}^{(l)}$, up to $\mathcal{C}+\tilde{\mathcal{C}}_{+}$, where $\mathcal{C}$ is the correction in trace norm in Eq. (14) and $\tilde{\mathcal{C}}_{+}$is given in Eq. (13) [83]. Here, $c_{k}^{(l)}=\operatorname{Tr}\left(L_{k} \rho_{l}\right)$ represent the metastable phases $\rho_{l}$ in the coefficient space [cf. Eq. (4)],

$$
\tilde{\rho}_{l} \equiv \mathcal{P}\left(\rho_{l}\right)=\rho_{\mathrm{ss}}+\sum_{k=2}^{m} c_{k}^{(l)} R_{k}, \quad l=1, \ldots, m .
$$

Thus, the MM is approximated by a simplex in the coefficient space with vertices given by the metastable phases. For lowdimensional MMs $(m \leqslant 4)$, this can be verified visually by projecting a randomly generated set of initial conditions on their metastable states (to sample the MM) and checking that they are found approximately within the chosen simplex (cf. Fig. 2).

Motivated by the structure of classical MMs in the coefficient space, we now introduce the test of classicality - a geometric way of checking whether degrees of freedom describing metastable states during the metastable regime correspond, approximately, to probability distributions. Degrees of freedom in the MM are described by the coefficients of decomposition into the eigenmodes $R_{k}, k=1, \ldots, m$, so that, with $c_{1}=1$, their number is $m-1$. Motivated by Eq. (14), here we instead consider the decomposition in the new basis given by the projections of metastable phases in Eq. (17), which is encoded by the transformation

$$
(\mathbf{C})_{k l} \equiv c_{k}^{(l)}, \quad k, l=1, \ldots, m,
$$

so that $\tilde{\rho}_{l}=\sum_{k=1}^{m}(\mathbf{C})_{k l} R_{k}$. In particular, the volume of the corresponding simplex in the coefficient space is $|\operatorname{det} \mathbf{C}| /$ $(m-1)$ ! [84]. The decomposition of a metastable state in this new basis [cf. Eq. (4)]

$$
\mathcal{P}[\rho(0)]=\sum_{l=1}^{m} \tilde{p}_{l} \tilde{\rho}_{l},
$$

is given by the barycentric coordinates $\tilde{p}_{l}=\sum_{k=1}^{m}\left(\mathbf{C}^{-1}\right)_{l k} c_{k}$ of the simplex in the coefficient space, so that $\tilde{p}_{l}=\operatorname{Tr}\left[\tilde{P}_{l} \rho(0)\right]$ with the new dual basis

$$
\tilde{P}_{l} \equiv \sum_{k=1}^{m}\left(\mathbf{C}^{-1}\right)_{l k} L_{k}, \quad l=1, \ldots, m .
$$

When $\tilde{\rho}_{l}$ are linearly independent for $l=1, \ldots, m,|\operatorname{det} \mathbf{C}|>$ 0 and $\mathbf{C}$ is invertible so that Eq. (20) is well defined. In this case, $\operatorname{Tr}\left(\tilde{P}_{k} \tilde{\rho}_{l}\right)=\sum_{n=1}^{m}\left(\mathbf{C}^{-1}\right)_{k n}(\mathbf{C})_{n l}=\left(\mathbf{C}^{-1} \mathbf{C}\right)_{k l}=\delta_{k l}$ and the normalization of the dual basis in Eq. (20) is fixed by the traces of metastable states in Eq. (17) being 1.

Although for the barycentric coordinates we have $\sum_{l=1}^{m} \tilde{p}_{l}=1$, and thus $\sum_{l=1}^{m} \tilde{P}_{l}=\mathbb{1}$, they do not in general correspond to probability distributions. Indeed, they are not all positive whenever a metastable state lies outside the simplex in the coefficient space corresponding to $\tilde{\rho}_{l}, l=1, \ldots, m$ (see Fig. 2), as the distance in L1 norm of barycentric coordinates to the simplex is given by $\|\tilde{\mathbf{p}}-\mathbf{p}\|_{1}=\|\tilde{\mathbf{p}}\|_{1}-1$, where $\mathbf{p}$ is the closest probability distribution to barycentric coordinates $\tilde{\mathbf{p}}\left[\right.$ here, $(\mathbf{p})_{l}=p_{l}$ and $\left.(\tilde{\mathbf{p}})_{l}=\tilde{p}_{l}, l=1, \ldots, m\right]$. Nevertheless, when the maximum distance to the simplex (cf. Fig. 2),

$$
\mathcal{C}_{\mathrm{cl}}\left(\tilde{\rho}_{1}, \ldots, \tilde{\rho}_{m}\right) \equiv \max _{\rho(0)}\|\tilde{\mathbf{p}}\|_{1}-1,
$$

is small, it follows that the metastability is classical, with the corrections in Eq. (14) bounded as

$$
\left\|\rho(t)-\sum_{l=1}^{m} p_{l} \rho_{l}\right\| \lesssim \mathcal{C}_{\mathrm{cl}}\left(\tilde{\rho}_{1}, \ldots, \tilde{\rho}_{m}\right)+\mathcal{C}_{+}+\mathcal{C}_{\mathrm{MM}},
$$

where $t^{\prime \prime} \leqslant t \leqslant t^{\prime}$ and $\lesssim$ stands for $\leqslant$ in the leading order of the corrections (see Sec. C2 in Ref. [55]), while $p_{l}$ is chosen as the closest probability distribution to the barycentric coordinates, $\rho_{l}$ as the closest state to $\tilde{\rho}_{l}$, so that $\left\|\tilde{\rho}_{l}-\rho_{l}\right\| \leqslant \mathcal{C}_{+}$ 
[cf. Eq. (12)], and $\mathcal{C}_{\mathrm{MM}}$ bounds the approximation of $\rho(t)$ by the projection on the low-lying modes [cf. Eqs. (10) and (19)]. Similarly, the average distance to the simplex can be considered (cf. Refs. [85,86] and see Sec. C2 in Ref. [55]).

Finally, the corrections in Eq. (21), which we refer to as the corrections to the classicality, can be efficiently estimated using the dual basis,

$$
\mathcal{C}_{\mathrm{cl}}\left(\tilde{\rho}_{1}, \ldots, \tilde{\rho}_{m}\right) \leqslant 2 \sum_{l=1}^{m}\left(-\tilde{p}_{l}^{\text {min }}\right) \equiv \tilde{\mathcal{C}}_{\mathrm{cl}}\left(\tilde{\rho}_{1}, \ldots, \tilde{\rho}_{m}\right),
$$

where $\tilde{p}_{l}^{\min } \leqslant 0$ is the minimum eigenvalue of $\tilde{P}_{l}$ in Eq. (20), so that

$$
\tilde{\mathcal{C}}_{\mathrm{cl}}\left(\tilde{\rho}_{1}, \ldots, \tilde{\rho}_{m}\right) \leqslant m \mathcal{C}_{\mathrm{cl}}\left(\tilde{\rho}_{1}, \ldots, \tilde{\rho}_{m}\right) .
$$

Apart from being easy to compute, $\tilde{\mathcal{C}}_{\mathrm{cl}}\left(\tilde{\rho}_{1}, \ldots, \tilde{\rho}_{m}\right)$ also carries the operational meaning of being an upper bound on the distance of the operators $\tilde{P}_{l}$ to the set of POVMs; cf. Sec. IV B and see Sec. D1 in Ref. [55].

\section{Figures of merit}

From Eq. (21), we obtain a criterion for verification of whether for a given set of states, the MM can be approximated as a probabilistic mixture of the corresponding metastable states [Eq. (17)]. In particular, whenever

$$
\mathcal{C}_{\mathrm{cl}}\left(\tilde{\rho}_{1}, \ldots, \tilde{\rho}_{m}\right) \ll 1,
$$

the metastability is classical. Moreover, it can be shown that $\quad \mathcal{C}_{\mathrm{cl}}\left(\tilde{\rho}_{1}, \ldots, \tilde{\rho}_{m}\right) \lesssim \mathcal{C}\left(\rho_{1}, \ldots, \rho_{m}\right)+\tilde{\mathcal{C}}_{+}, \quad$ provided that $m\left[\mathcal{C}\left(\rho_{1}, \ldots, \rho_{m}\right)+\tilde{\mathcal{C}}_{+}\right] \ll 1$, which also implies $\tilde{\mathcal{C}}_{\mathrm{cl}}\left(\tilde{\rho}_{1}, \ldots, \tilde{\rho}_{m}\right) \ll 1$ (cf. Eqs. (15) and (23), and see Sec. C2 in Ref. [55]). Since for the classical metastability we have Eq. (16), assuming these corrections decrease when changing a dynamical parameter or when increasing system size, but $m$ remains constant, Eq. (25) follows. We thus conclude that it is a necessary and sufficient condition for classical metastability.

Interestingly, the bimodal case of $m=2$ is always classical as the metastable phases $\tilde{\rho}_{1}$ and $\tilde{\rho}_{2}$ leading to $\mathcal{C}_{\mathrm{cl}}\left(\tilde{\rho}_{1}, \tilde{\rho}_{2}\right)=$ $\tilde{\mathcal{C}}_{\mathrm{cl}}\left(\tilde{\rho}_{1}, \tilde{\rho}_{2}\right)=0$ can be constructed explicitly [40,41]. For higher dimensional MMs, the presence of classical metastability can be uncovered using the bound in Eq. (23) for candidate states generated by the numerical approaches of Sec. VII, see, e.g., Ref. [54]. In particular, approaching an effectively classical first-order dissipative phase transition with a finite $m$ requires the possibility of choosing $m$ candidate states such that $\mathcal{C}_{\mathrm{cl}}\left(\tilde{\rho}_{1}, \ldots, \tilde{\rho}_{m}\right) \rightarrow 0$ (cf. Sec. IID). Importantly, the condition in Eq. (25) is independent from the presence of weak symmetries (which, nevertheless, can be efficiently incorporated; cf. Sec. VI B 5).

In Sec. IV, we show that the metastable phases in Eq. (17) are approximately disjoint, while the operators in Eq. (20) take the role of basins of attractions. Moreover, in Sec. V, we explain how the long-time dynamics toward the stationary state corresponds approximately to classical stochastic dynamics between metastable phases. Since corrections in those results depend only on the corrections to the stationarity, the positivity, and the classicality defined in Eqs. (10), (12), and (21), these quantities can be viewed as a complete set of figures of merit characterizing classical metastability in open quantum systems. As in the most of this work, we consider a given choice of $m$ states, we denote $\mathcal{C}\left(\rho_{1}, \ldots, \rho_{m}\right)$ by $\mathcal{C}$ and $\mathcal{C}_{\mathrm{cl}}\left(\tilde{\rho}_{1}, \ldots, \tilde{\rho}_{m}\right)$ by $\mathcal{C}_{\mathrm{cl}}$ for simplicity.

\section{CLASSICAL METASTABLE PHASES}

In Sec. III, we introduced the definition of classical metastability of when MMs of open quantum systems can be approximated as probabilistic mixtures of a set of states. We now show that in this case, those states are necessarily metastable and constitute a physical basis of the MM as distinct phases of the system. To this aim, we demonstrate that the probabilities that represent the degrees of freedom in the MM can be accessed with negligible disturbance, so that the metastable phases can be distinguished with a negligible error. We further argue that their supports and basins of attraction are approximately disjoint, in analogy to first-order phase transitions and metastability in classical Markovian systems [38]. Finally, we also discuss how, in the case of any further separation in the low-lying spectrum, later MMs are necessarily classical as well.

\section{A. Physical representation of metastable manifold}

We begin by noting that phases given in Eq. (14) are uniquely defined up to the so far considered corrections when the condition in Eq. (16) is fulfilled. Indeed, for states $\rho_{l}$ in Eq. (14), the distance to their projections $\tilde{\rho}_{l}$ in Eq. (17) is bounded by $2\left(\mathcal{C}+\tilde{\mathcal{C}}_{+}\right)$when $\tilde{\mathcal{C}}_{\mathrm{cl}} \ll 1$ (see Sec. $\mathrm{C} 2$ in Ref. [55]), so that they are metastable. It then follows that their distance to the states chosen in Eq. (22) as closest states to the projections $\tilde{\rho}_{l}$ in Eq. (17) is bounded by $\lesssim 2(\mathcal{C}+$ $\left.\tilde{\mathcal{C}}_{+}\right)+\mathcal{C}_{+}$. Finally, for two different sets of $m$ metastable phases corresponding to different projections in Eq. (17) and the corrections to the classicality $\mathcal{C}_{\mathrm{cl}}$ and $\mathcal{C}_{\mathrm{cl}}^{\prime}$, which fulfill Eq. (25), the distance in trace norm between the projection of a metastable phase in one set to the closest projection of a metastable phase in the other set is bounded by $\lesssim \mathcal{C}_{\mathrm{cl}}+\mathcal{C}_{\mathrm{cl}}^{\prime}+$ $\min \left(\mathcal{C}_{\mathrm{cl}}, \mathcal{C}_{\mathrm{cl}}^{\prime}\right)$ (see Sec. D in Ref. [55])

Furthermore, in contrast to the right eigenmodes of the master operator with $\operatorname{Tr}\left(R_{k}\right)=0$ for $k=2, \ldots, m$ [from $L_{1}=$ $\mathbb{1}$ and $\left.\operatorname{Tr}\left(L_{k} R_{l}\right)=\delta_{k l}\right]$, the projections of metastable phases in Eq. (17) feature normalized trace, $\operatorname{Tr}\left(\tilde{\rho}_{l}\right)=1, l=1, \ldots, m$, are Hermitian, and approximately positive [see Sec. II A and cf. Eq. (12)]. Moreover, when the condition in Eq. (25) is fulfilled, any metastable state is approximated well by their probabilistic mixture [cf. Eq. (22) and Fig. 2]. Thus, the projections in Eq. (17) can be considered as physical basis of the MM and approximate metastable phases.

While the left low-lying eigenmodes $L_{k}, k=2, \ldots, m$, describe quantities conserved in the system during the initial relaxation and the metastable regime [cf. Eq. (8) for $t \leqslant t^{\prime}$, where $b_{k}=\delta_{k l}$ for $O=L_{l}$ ] the dual basis operators in Eq. (20) determine the decomposition of a metastable state into the basis in Eq. (17), and as such, when the condition in Eq. (25) is fulfilled, they represent approximate basins of attraction for metastable phases (see also Sec. D1 in Ref. [55]). Importantly, via barycentric coordinates in Eq. (35), they define order parameters that distinguish the metastable phases, 
$\operatorname{Tr}\left(\tilde{P}_{k} \tilde{\rho}_{l}\right)=\delta_{k l}$, with system observable averages being their linear combinations [cf. Eq. (8)].

Finally, the barycentric coordinates are a physical representation of $m$-1 degrees of freedom present in the metastable regime, as they approximate probability distributions. As a consequence, we next show that they are classical from an operational perspective of measuring the system.

\section{B. Classical degrees of freedom}

We now argue that in the case of classical metastability, the degrees of freedom determining the MM can be accessed with a negligible disturbance of metastable states. It then follows that metastable phases can be distinguished with a negligible error. We also discuss consequences for measurements of system observables.

The MM is determined by the coefficients $c_{k}$ of decomposition into low-lying eigenmodes $L_{k}, k=2, \ldots, m$ [cf. Eq. (4)], or, equivalently, by the barycentric coordinates $\tilde{p}_{l}$ of decomposition into the projections $\tilde{\rho}_{l}$ of metastable phases, $l=1, \ldots, m$ [cf. Eqs. (17) and (19)]. Furthermore, system states can be probed by POVMs $\left(P_{l}=P_{l}^{\dagger}, P_{l} \geqslant 0, \sum_{l} P_{l}=\right.$ $\mathbb{1})$, including von Neumann measurements $\left(P_{k} P_{l}=\delta_{k l} P_{l}\right)$ corresponding to measuring system observables. Considering the POVM

$$
P_{l} \equiv \frac{\tilde{P}_{l}-\tilde{p}_{l}^{\min } \mathbb{1}}{1+\frac{\tilde{\mathcal{C}}_{\mathrm{cl}}}{2}}, \quad l=1, \ldots, m,
$$

the distance of the probability distribution $(\mathbf{p})_{l} \equiv \operatorname{Tr}\left[P_{l} \rho(0)\right]$, $l=1, \ldots, m$, to the barycentric coordinates is bounded by $\|\tilde{\mathbf{p}}-\mathbf{p}\|_{1} \lesssim \tilde{\mathcal{C}}_{\mathrm{cl}}$ (cf. Eq. (23) and see Sec. D1 in Ref. [55]). Therefore, by measuring a metastable state $\rho(t)$, the barycentric coordinates can be accessed with the error up to $\tilde{\mathcal{C}}_{\mathrm{cl}}+\mathcal{C}_{\mathrm{MM}}$ [cf. Eq. (10)], while the state can be reconstructed by preparing the closest state $\rho_{l}$ to $\tilde{\rho}_{l}$ upon obtaining $l$ th outcome, with the resulting disturbance [87]

$$
\left\|\rho(t)-\sum_{l=1}^{m} \operatorname{Tr}\left[P_{l} \rho(t)\right] \rho_{l}\right\| \lesssim \tilde{\mathcal{C}_{\mathrm{cl}}}+\mathcal{C}_{+}+\mathcal{C}_{\mathrm{MM}},
$$

where $t^{\prime \prime} \leqslant t \leqslant t^{\prime}$. This result should be contrasted with the case of measuring a general system state where no information is available without disturbance (see, e.g., Ref. [88]).

The minimal average error of distinguishing equally probable two states according to Holevo-Helstrom theorem is determined by the distance in the trace norm as $1 / 2-\| \rho^{(1)}-$ $\rho^{(2)} \| / 4$ (see, e.g., Ref. [89]). For metastable states, this error is approximately determined by the distance between their barycentric coordinates as

$$
\begin{aligned}
\left\|\rho^{(1)}(t)-\rho^{(2)}(t)\right\| & \gtrsim\left\|\tilde{\mathbf{p}}^{(1)}-\tilde{\mathbf{p}}^{(2)}\right\|_{1}\left(1-\frac{\tilde{\mathcal{C}}_{\mathrm{cl}}}{2}\right)-2 \mathcal{C}_{\mathrm{MM}}, \\
\left\|\rho^{(1)}(t)-\rho^{(2)}(t)\right\| & \lesssim\left\|\tilde{\mathbf{p}}^{(1)}-\tilde{\mathbf{p}}^{(2)}\right\|_{1}\left(1+\mathcal{C}_{+}\right)+2 \mathcal{C}_{\mathrm{MM}},
\end{aligned}
$$

where the first bound corresponds to the error when measuring the POVM in Eq. (26). In particular, a pair of metastable phases can be distinguished with the error $\lesssim\left[\min \left(\tilde{\mathcal{C}}_{\mathrm{cl}} / 2, \mathcal{C}_{\mathrm{cl}}\right)+\mathcal{C}_{+}\right] / 2$, since

$$
\left\|\rho_{k}-\rho_{l}\right\| \gtrsim 2\left(1-\mathcal{C}_{\mathrm{cl}}-\mathcal{C}_{+}\right), \quad k \neq l,
$$

for $\rho_{l}$ being the closest state to $\tilde{\rho}_{l}$ in Eq. (17), which corresponds to a measuring the POVM with two elements: $P \equiv$ $\left(\tilde{P}_{l}-\tilde{p}_{l}^{\min } \mathbb{1}\right) /\left(\tilde{p}_{l}^{\max }-\tilde{p}_{l}^{\min }\right)$ and $\mathbb{1}-P$. For $\rho_{l}$ that projects on $\tilde{\rho}_{l}$, the bound in Eq. (29) reduces to $\gtrsim 2\left(1-\mathcal{C}_{\mathrm{cl}}\right)$. For derivations, see Sec. D2 in Ref. [55].

Finally, during the metastable regime, $t^{\prime \prime} \leqslant t \leqslant t^{\prime}$, the probability distribution for any measurement of the system is approximated as a probabilistic mixture of probability distribution for individual metastable phases, as

$$
\sum_{k}\left|\operatorname{Tr}\left[P_{k} \rho(t)\right]-\sum_{l=1}^{m} p_{l} \operatorname{Tr}\left[P_{k} \rho_{l}\right]\right| \leqslant\left\|\rho(t)-\sum_{l=1}^{m} p_{l} \rho_{l}\right\|,
$$

where $\left\{P_{k}\right\}_{k}$ is a POVM and the right-hand side is bounded by corrections in Eqs. (14) or (22), depending on the choice of metastable phases. This conditional structure of the probability distribution, however, is not directly related to the classicality, since it is present for any system state being a probabilistic mixture of not necessarily orthogonal states, while, as we argue next, metastable phases are approximately disjoint.

\section{Approximate disjointness of metastable phases}

Below we show that the metastable phases in Eqs. (14) and (22) are approximately disjoint, that is, they describe states restricted to distinct regions of the system space. Furthermore, we also find that their basins of attraction are approximately disjoint.

First, note that the distance in the trace norm equals 2 only for disjoint (mutually orthogonal, states). Therefore, the bound in Eq. (29) implies that the metastable phases are approximately disjoint. This is further corroborated by similar bounds on scalar products of $\sqrt{\rho_{l}}$ or $\rho_{l}$; see Sec. D3 of Ref. [55].

Second, to capture approximately disjoint supports of metastable phases, we consider the subspaces $\mathcal{H}_{l}$ defined as the space spanned by eigenstates of $\tilde{P}_{l}$ in Eq. (20) with eigenvalues equal or above $1 / 2, l=1, \ldots, m$. We have (see Sec. D3 in Ref. [55])

$$
\begin{gathered}
\operatorname{Tr}\left(\mathbb{1}_{\mathcal{H}_{k}} \rho_{l}\right) \lesssim \mathcal{C}_{\mathrm{cl}}+2 \mathcal{C}_{+}, \quad k \neq l, \\
\operatorname{Tr}\left(\mathbb{1}_{\mathcal{H}_{l}} \rho_{l}\right) \gtrsim 1-\mathcal{C}_{\mathrm{cl}}-2 \mathcal{C}_{+},
\end{gathered}
$$

where $\rho_{l}$ is the closest state to $\tilde{\rho}_{l}, k, l=1, \ldots, m$. Furthermore, we also have [cf. Eq. (23)]

$$
\sum_{\substack{1 \leqslant k \leqslant m: \\ k \neq l}} \operatorname{Tr}\left(\mathbb{1}_{\mathcal{H}_{k}} \rho_{l}\right) \lesssim \tilde{\mathcal{C}}_{\mathrm{cl}}+2 \mathcal{C}_{+}
$$

The bounds in Eqs. (31)-(33) support the statement that the metastable phases reside in approximately disjoint areas of the state space. The bounds in Eqs. (31)-(33) also hold well for $\tilde{\rho}_{l}$ in Eq. (17) as $\left|\operatorname{Tr}\left(\mathbb{1}_{\mathcal{H}_{k}} \rho_{l}\right)-\operatorname{Tr}\left(\mathbb{1}_{\mathcal{H}_{k}} \tilde{\rho}_{l}\right)\right| \leqslant \mathcal{C}_{+}$, while for the states in Eq. (14) that project on $\tilde{\rho}_{l}$, they are further reduced to $\lesssim \mathcal{C}_{\mathrm{cl}}, \gtrsim 1-\mathcal{C}_{\mathrm{cl}}$ and $\lesssim \tilde{\mathcal{C}}_{\mathrm{cl}}$, respectively. For the bimodal case of open quantum dynamics, $m=2$, approximate disjointness was already argued in Refs. [40,41]. 
Finally, the subspace $\mathcal{H}_{l}$ in Eqs. (31)-(33) captures not only majority of $\rho_{l}$ support, but by its definition also the corresponding basin of attraction, i.e., the initial states which evolve into metastable states close to $\tilde{\rho}_{l}$, i.e., with $1-\tilde{p}_{l} \ll 1$. Indeed, in Sec. D3 of Ref. [55], we show that $\operatorname{Tr}\left[\mathbb{1}_{\mathcal{H}_{l}} \rho(0)\right] \gtrsim$ $1-2\left|1-\tilde{p}_{l}\right|-\mathcal{C}_{\mathrm{cl}}$. Furthermore, we have $\operatorname{Tr}\left[\mathbb{1}_{\mathcal{H}_{k}} \rho(0)\right] \leqslant$ $2\left|1-\tilde{p}_{l}\right|+\mathcal{C}_{\mathrm{cl}}$ for $k \neq l$, and $\sum_{1 \leqslant k \leqslant m: k \neq l} \operatorname{Tr}\left[\mathbb{1}_{\mathcal{H}_{k}} \rho(0)\right] \leqslant$ $2\left|1-\tilde{p}_{l}\right|+\tilde{\mathcal{C}}_{\mathrm{cl}}$. Thus, we conclude that the basins of attractions are approximately disjoint. We note, however, that in general subspaces $\mathcal{H}_{l}$ themselves are not disjoint as they feature states that decay into multiple metastable phases (it can be shown that subspaces spanned by the $\tilde{P}_{l}$ eigenstates with the eigenvalues separated from 0 and 1 by distance $\gg \mathcal{C}_{\mathrm{cl}}+\mathcal{C}_{+}$, $l=1, \ldots, m$, can be neglected in the support of metastable phases; see Sec. D3 in Ref. [55]).

\section{Classical hierarchy of metastable phases}

A second metastable regime corresponds to a further separation in the low-lying spectrum of the master operator in Eq. (1), $\lambda_{m_{2}}^{R} / \lambda_{m_{2}+1}^{R} \ll 1$ with $m_{2}<m$ (cf. Ref. [90]). In Sec. F of Ref. [55], we show that metastable states during the second metastable regime, $t_{2}^{\prime \prime} \leqslant t \leqslant t_{2}^{\prime}$, also form a classical MM, i.e., are mixtures of $m_{2}$ metastable phases provided that $t_{2}^{\prime} \geqslant 2 t_{2}^{\prime \prime}$. Those $m_{2}$ metastable phases are approximately disjoint mixtures of $m$ metastable phases of the first MM, and their supports as well as their basins of attraction are approximately disjoint [cf. Eqs. (29)-(33)]. Therefore, each metastable phase of the first MM evolves approximately into a single metastable phase in the second MM, unless the second $\mathrm{MM}$ is not supported on that phase (the phase belongs to the decay subspace).

These results are a direct consequence of long-time dynamics in a classical MM being well approximated by classical stochastic dynamics, which we discuss in next, as metastable states of classical stochastic dynamics are known to be mixtures of as many metastable phases as the number of low-lying modes [36,38].

\section{CLASSICAL LONG-TIME DYNAMICS}

The definition of classical metastability in Eq. (14) determines not only the structure of metastable states. Remarkably, as a consequence of the long-time relaxation toward the stationary state effectively taking place inside the MM, the long-time dynamics is approximately classical as well. We prove that it corresponds to classical stochastic dynamics occurring between disjoint metastable phases and can be accessed by measuring averages or time-correlations of system observables whenever metastable phases differ in the averages. We also discuss the role played by a further separation of timescales in the long-time dynamics, i.e., another metastable regime. Finally, we show that stochastic transitions between metastable phases can be observed directly by means of continuous measurements of quanta emitted during system interaction with the environment, provided that metastable phases differ in the average measurement rates. In that case, the statistics of integrated continuous measurement is generally multimodal for times within the metastable regime, which for times after the final relaxation can lead to a high fluctuations rate, reminiscent of the proximity to a dynamical phase transition [60].

\section{A. Classical average dynamics of system and observables}

\section{Long-time dynamics}

From Eq. (5) the evolution for times $t \geqslant t^{\prime \prime}$ effectively takes place on the MM with the effective generator $\mathcal{L}_{\mathrm{MM}}$ defined in Eq. (6). This generator can be expressed in the basis of the metastable phases [Eqs. (17) and (20)] as

$$
(\tilde{\mathbf{W}})_{k l} \equiv \operatorname{Tr}\left[\tilde{P}_{k} \mathcal{L}_{\mathrm{MM}}\left(\tilde{\rho}_{l}\right)\right],
$$

where $k, l=1, . ., m$, and thus $\tilde{\mathbf{W}}=\mathbf{C}^{-1} \boldsymbol{\Lambda} \mathbf{C}$ with $(\boldsymbol{\Lambda})_{k l} \equiv$ $\lambda_{k} \delta_{k l}$ [cf. Eq. (18) and see Fig. 3(a)]. The dynamics of the system state within the MM is then determined by the dynamics of the barycentric coordinates

$$
\tilde{\mathbf{p}}(t)=e^{t \tilde{\mathbf{W}}} \tilde{\mathbf{p}},
$$

where $(\tilde{\mathbf{p}})_{l} \equiv \operatorname{Tr}\left[\tilde{P}_{l} \rho(0)\right]$, so that $\mathcal{P}[\rho(t)]=\sum_{l=1}^{m}[\tilde{\mathbf{p}}(t)]_{l} \tilde{\rho}_{l}$ [cf. Eq. (19) and see Fig. 3(b)].

By definition, the long-time evolution in Eq. (6) transforms the MM onto itself, see, e.g., Fig. 1(b). This does not guarantee, however, that the simplex of $m$ metastable phases is transformed onto itself, as the evolution may cause states inside the simplex to evolve toward states outside, and thus an initial probability distribution (positive barycentric coordinates) acquiring some negative values at later times [see the inset in Fig. 3(b)]. Therefore, the dynamics generated by $\tilde{\mathbf{W}}$ is in general not positive [cf. Fig. 3(a)]. Nevertheless, as we discuss below, when the simplex of metastable phases is a good approximation for the MM in the sense of the condition in Eq. (25), $\tilde{\mathbf{W}}$ is well approximated by a generator of stochastic classical dynamics between metastable phases.

\section{Classical generator}

Dynamics generated by $\tilde{\mathbf{W}}$ conserves the probability, as from $\sum_{k=1}^{m} \tilde{P}_{k}=\mathbb{1}$ we have $\sum_{k=1}^{m}(\tilde{\mathbf{W}})_{k l}=0$ (cf. Sec. E1 in Ref. [55]). Furthermore, it can be shown to be approximately positive, with $\tilde{\mathbf{W}}$ approximated by the closest classical stochastic generator W (cf. Fig. 3(a) and see Secs. E2a and E2b in Ref. [55]),

$$
\begin{aligned}
(\mathbf{W})_{k l} & \equiv \max \left[(\tilde{\mathbf{W}})_{k l}, 0\right], \quad k \neq l, \\
(\mathbf{W})_{l l} & \equiv(\tilde{\mathbf{W}})_{l l}+\sum_{k \neq l} \min \left[(\tilde{\mathbf{W}})_{k l}, 0\right],
\end{aligned}
$$

$k, l=1, \ldots, m$, as

$$
\frac{\|\tilde{\mathbf{W}}-\mathbf{W}\|_{1}}{\|\tilde{\mathbf{W}}\|_{1}} \lesssim 2 \sqrt{\mathcal{C}_{\mathrm{cl}}},
$$

where the norm $\|\mathbf{X}\|_{1} \equiv \max _{1 \leqslant l \leqslant m} \sum_{k=1}^{m}\left|(\mathbf{X})_{k l}\right|$ [91] and $\left(1-\mathcal{C}_{+}-\mathcal{C}_{\mathrm{cl}}\right)\left\|\mathcal{L}_{\mathrm{MM}}\right\| \lesssim\|\tilde{\mathbf{W}}\|_{1} \leqslant\left(1+\tilde{\mathcal{C}}_{\mathrm{cl}} / 2\right)\left\|\mathcal{L}_{\mathrm{MM}}\right\| \quad($ see Sec. D2 of in Ref. [55]). From Eq. (37) the normalized distance between the generators is bounded as

$$
\Delta_{+} \equiv \frac{\|\tilde{\mathbf{W}}-\mathbf{W}\|_{1}}{\|\tilde{\mathbf{W}}\|_{1}+\|\mathbf{W}\|_{1}} \lesssim \sqrt{\mathcal{C}_{\mathrm{cl}}} .
$$




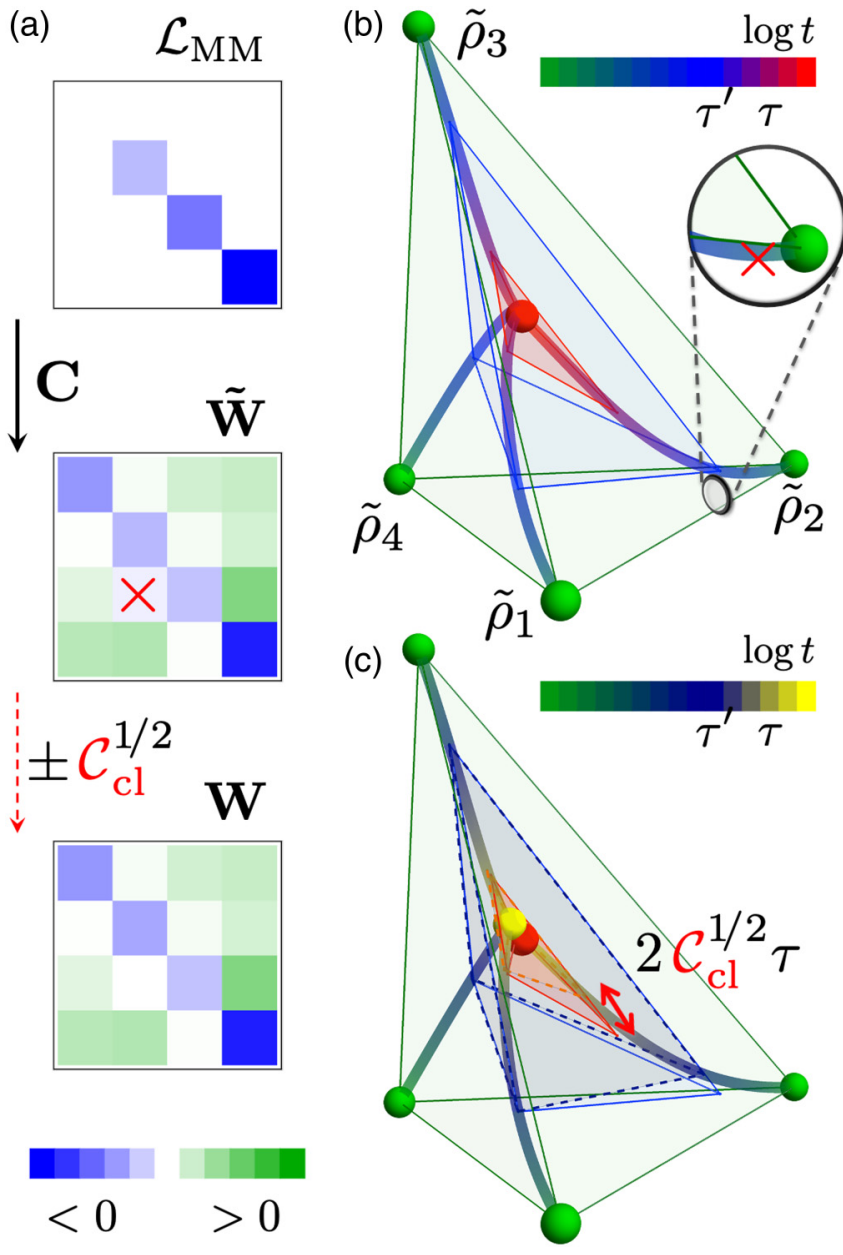

FIG. 3. Classical long-time dynamics: (a) The long-time dynamics [Eq. (5)] can be understood as dynamics between metastable phases [Eq. (35)], governed by the trace-preserving generator $\tilde{\mathbf{W}}$ [Eq. (34)], which can be approximated by a classical stochastic generator $\mathbf{W}$ [Eqs. (36) and (37)], which is both trace preserving and positive; here a negative transition rate from $\tilde{\rho}_{2}$ to $\tilde{\rho}_{3}$ (marked by red cross) is put to 0 . (b) The long-time dynamics in the barycentric coordinates (cf. Fig. 2): green simplex corresponds to $t \ll \tau^{\prime}$, blue to $t=\tau^{\prime}$, and red to $t=\tau$, while the stationary state is marked by red sphere.Positive dynamics corresponds to the simplex of metastable phases mapped onto itself, which requires all metastable phases to be mapped inside the simplex at all times. Here, $\tilde{\rho}_{2}$ initially acquires a negative probability $\tilde{p}_{3}(t)$ at small $t$ [red cross in the inset; cf. $\tilde{\mathbf{W}}$ in panel (a)]. (c) Approximating by $\mathbf{W}$ alters the dynamics, with corrections increasing in time [Eq. (39)]; blue dashed simplex corresponds to $t=\tau^{\prime}$ and orange dashed to $t=\tau$ [cf. panel (b)]. This ultimately leads to a different stationary state (yellow sphere) [cf. Eq. (41)], which is close to the true stationary state when Eq. (40) is fulfilled.

Note that columns of $\mathbf{W}$ sum to 0 , and then negativity of its diagonal terms follows from the positivity of the off-diagonal terms, so that dynamics generated by $\mathbf{W}$ is indeed positive and probability-conserving (cf. Sec. E1 in Ref. [55]). For the bimodal case of $m=2$, the MM is always classical with $\mathcal{C}_{\mathrm{cl}}=0$, and thus $\tilde{\mathbf{W}}=\mathbf{W}$ is exactly a generator of stochastic classical dynamics [41].

\section{Classical system dynamics}

We now discuss how the dynamics generated by $\tilde{\mathbf{W}}$ is approximated by the classical dynamics generated by $\mathbf{W}$. We also discuss conditions for the stationary state to be approximated in terms of stationary distribution of $\mathbf{W}$.

In Sec. E2c of Ref. [55], we show it follows from Eq. (37) that

$$
\left\|e^{t \tilde{\mathbf{W}}}-e^{t \mathbf{W}}\right\|_{1} \lesssim 2 \sqrt{\mathcal{C}_{\mathrm{cl}}} t\|\tilde{\mathbf{W}}\|_{1} .
$$

Therefore, for times $t\|\tilde{\mathbf{W}}\|_{1} \ll 1 / \sqrt{\mathcal{C}_{\mathrm{cl}}}$ the effective dynamics in the MM is well approximated by the classical dynamics, as $\|\tilde{\mathbf{p}}(t)-\mathbf{p}(t)\|_{1} \leqslant\left\|e^{t \tilde{\mathbf{W}}}-e^{t \mathbf{W}}\right\|_{1}\|\tilde{\mathbf{p}}\|_{1} \lesssim\left\|e^{t \tilde{\mathbf{W}}}-e^{t \mathbf{W}}\right\|_{1}$ [where $\mathbf{p}(t)=e^{t \mathbf{W}} \tilde{\mathbf{p}}$, cf. Eq. (35); $\tilde{\mathbf{p}}$ can further replaced by the closest probability distribution with additional corrections bounded by $\mathcal{C}_{\mathrm{cl}}$, which are of the higher-order for times after the metastable regime, e.g., $\left.t \geqslant 1 /\|\tilde{\mathbf{W}}\|_{1}\right]$; see Fig. 3(c). This also holds true for the corresponding density matrices (see Sec. D2 of Ref. [55]).

When the approximation in Eq. (39) holds for times after the relaxation in the $\mathrm{MM}$, which requires

$$
\tau\|\tilde{\mathbf{W}}\|_{1} \ll \frac{1}{\sqrt{\mathcal{C}_{\mathrm{cl}}}},
$$

the stationary state $\rho_{\mathrm{ss}}$ described within the MM by $\left(\tilde{\mathbf{p}}_{\mathrm{ss}}\right)_{k}=$ $\operatorname{Tr}\left(\tilde{P}_{k} \rho_{\mathrm{ss}}\right)$ is well approximated by the stationary probability $\mathbf{p}_{\mathrm{ss}}$ of the classical dynamics W [92]; cf. Fig. 3(c). Indeed,

$$
\left\|\tilde{\mathbf{p}}_{\mathrm{ss}}-\mathbf{p}_{\mathrm{ss}}\right\|_{1} \lesssim\left\|\tilde{\mathbf{P}}_{\mathrm{ss}}-e^{t \tilde{\mathbf{W}}_{\|}}\right\|_{1}+2 \sqrt{\mathcal{C}_{\mathrm{cl}}} t\|\tilde{\mathbf{W}}\|_{1},
$$

where $\tilde{\mathbf{P}}_{\mathrm{ss}}$ denotes the projection on $\tilde{\mathbf{p}}_{\mathrm{ss}}$. Therefore, $\| \tilde{\mathbf{p}}_{\mathrm{ss}}-$ $\mathbf{p}_{\mathrm{ss}} \|_{1} \ll 1$ follows provided that $t\|\tilde{\mathbf{W}}\|_{1} \ll 1 / \sqrt{\mathcal{C}_{\mathrm{cl}}}$ for $t$ such that $\left\|\tilde{\mathbf{P}}_{\mathrm{ss}}-e^{t \tilde{\mathbf{W}}_{\|}}\right\|_{1} \ll 1$ [93]. As a corollary of Eq. (41),the stationary probability distribution $\mathbf{p}_{\mathrm{ss}}$ of classical dynamical generator $\mathbf{W}$ in Eq. (36) is unique. Thus, the classical dynamics is ergodic with the average time spent in $l$ th metastable phase equal $\left(\mathbf{p}_{\mathrm{ss}}\right)_{l}, l=1, \ldots, m$. Furthermore, the approximation also holds true for the distance in the trace norm of the corresponding density matrices (see Sec. D2 in Ref. [55]).

Similarly, not only the stationary state but all eigenmodes of the long-time dynamics in the MM can be approximated by those of the classical stochastic dynamics. In particular, in Sec. E2e of Ref. [55], we discuss approximation of the pseudoinverse of $\tilde{\mathbf{W}}$ in Eq. (34) by the pseudoinverse of $\mathbf{W}$ in Eq. (36), a result which plays an important role in the approximation of quantum trajectory statistics that we discuss in Sec. V B.

We note that the quality of the classical approximations for the structure of the long-time dynamics depends not only on the corrections $\mathcal{C}_{\mathrm{cl}}$ within the metastability regime [Eq. (21)], but also on the timescale of the final relaxation (cf. Eq. (41) and Secs. E2d and E2e in Ref. [55]). This is due to the fact that the approximation in Eq. (37) captures the fastest among the low-lying modes, while the final relaxation timescale is governed by the slowest among them. In particular, in the case of another metastable regime [90], which corresponds to further separation in the spectrum of the master operator in Eq. (1), the condition in Eq. (40) may generally not be valid. For example, when a classical first-order phase transition occurs at finite system size, in its proximity $1 /\left(\tau\|\tilde{\mathbf{W}}\|_{1}\right)$ is finite when the degeneracy of $m$ stable phases is lifted in the same 
order $1 /\left(\tau\|\tilde{\mathbf{W}}\|_{1}\right)$, so that time $t$ can be chosen $\tau\|\tilde{\mathbf{W}}\|_{1} \ll$ $t\|\tilde{\mathbf{W}}\|_{1} \ll 1 / \sqrt{\mathcal{C}_{\mathrm{cl}}}$ leading to $\left\|\tilde{\mathbf{p}}_{\mathrm{ss}}-\mathbf{p}_{\mathrm{ss}}\right\|_{1} \ll 1$. But when the perturbation away from the transition lifts the degeneracy of $m$ phases in several different orders (so that $\mathcal{C}_{\mathrm{cl}}$ is of a lower order in the perturbation than $1 / \tau)$, Eq. (40) is no longer fulfilled (see Sec. A2 in Ref. [55]). We discuss next how the approximation of the long-time dynamics by classical stochastic dynamics can be refined to take into account the hierarchy of metastabilities.

Finally, we note it is also possible to approximate $e^{t \tilde{\mathbf{W}}}$ by discrete classical dynamics. This again leads to corrections scaling linearly in time, but proportional to $\mathcal{C}_{\mathrm{cl}}$ rather than $\sqrt{\mathcal{C}_{\mathrm{cl}}}$; see Sec. E2f in Ref. [55].

\section{Hierarchy of classical long-time dynamics}

When there exists a second metastable regime in the system dynamics, $t_{2}^{\prime \prime} \leqslant t \leqslant t_{2}^{\prime}$, the corresponding metastable states of the system are simply approximated by the projection on the low-lying modes of the classical stochastic dynamics in Eq. (36) provided that $t_{2}^{\prime \prime} \ll 1 / \sqrt{\mathcal{C}_{\mathrm{cl}}}$ [cf. Eqs. (4) and (39)]. When, $t_{2}^{\prime} \geqslant 2 t_{2}^{\prime \prime}$, after the second metastable regime, $t \geqslant t_{2}^{\prime}$, the system dynamics toward the stationary state is approximated by classical dynamics taking place only between $m_{2}$ metastable phases of the second MM [cf. Eqs. (4) and (39), and see Sec. IV D]. Moreover, when that approximation holds also after the final relaxation, the system stationary state $\rho_{\mathrm{ss}}$ is well approximated by the stationary distribution of that classical dynamics [cf. Eq. (41)]. For further discussion, see Sec. F in Ref. [55].

\section{Classical observable dynamics}

We now argue how at times after the initial relaxation, the classical long-time dynamics can be observed in the behavior of expectation values or autocorrelations of general system observables. In particular, it can be directly accessed by measuring the dual basis in Eq. (20).

For times $t \geqslant t^{\prime \prime}$, the dynamics of the average for an observable $O$ depends only on the evolution of the distribution between the metastable phases,

$$
\begin{aligned}
\langle O(t)\rangle & =\tilde{\mathbf{o}}^{T} e^{t \tilde{\mathbf{W}}} \tilde{\mathbf{p}}+\cdots=\tilde{\mathbf{o}}^{T} \tilde{\mathbf{p}}(t)+\cdots \\
& =\tilde{\mathbf{o}}^{T} e^{t \mathbf{W}} \tilde{\mathbf{p}}+\cdots=\tilde{\mathbf{o}}^{T} \mathbf{p}(t)+\cdots,
\end{aligned}
$$

where $(\tilde{\mathbf{o}})_{l}=\operatorname{Tr}\left(O \tilde{\rho}_{l}\right), l=1, \ldots, m$, are the averages of the observable $O$ in the metastable phases. The first line corresponds to Eq. (8), while the second line follows from Eq. (39) introducing additional corrections bounded in the leading order by $2 t\|\tilde{\mathbf{W}}\|_{1} \sqrt{\mathcal{C}_{\mathrm{cl}}} \max _{1 \leqslant l \leqslant m}\left|(\tilde{\mathbf{o}})_{l}\right|$.

Similarly, the autocorrelation

$$
\begin{aligned}
\langle O(t) O(0)\rangle_{\mathrm{ss}}-\langle O\rangle_{\mathrm{ss}}^{2} & =\tilde{\mathbf{o}}^{T} e^{t \tilde{\mathbf{W}}} \tilde{\mathbf{O}} \tilde{\mathbf{p}}_{\mathrm{ss}}-\left(\tilde{\mathbf{o}}^{T} \tilde{\mathbf{p}}_{\mathrm{ss}}\right)^{2}+\cdots \\
& =\tilde{\mathbf{o}}^{T} e^{t \mathbf{W}} \tilde{\mathbf{O}} \mathbf{p}_{\mathrm{ss}}-\left(\tilde{\mathbf{o}}^{T} \mathbf{p}_{\mathrm{ss}}\right)^{2}+\cdots,
\end{aligned}
$$

where $(\tilde{\mathbf{O}})_{k l}=\operatorname{Tr}\left[\tilde{P}_{k} \mathcal{O}\left(\tilde{\rho}_{l}\right)\right]$ (cf. Ref. [41]). The first line corresponds to Eq. (9), while the second line follows from Eq. (39) with the additional corrections bounded by $\max _{1 \leqslant l \leqslant m} \mid(\tilde{\mathbf{o}})_{l}\left[\mid\|\tilde{\mathbf{O}}\|_{1}\left(2 t\|\tilde{\mathbf{\mathbf { W }}}\|_{1} \sqrt{\mathcal{C}_{\mathrm{cl}}}+\left\|\tilde{\mathbf{p}}_{\mathrm{ss}}-\mathbf{p}_{\mathrm{ss}}\right\|_{1}\right)+\right.$ $\left.2\left|\langle O\rangle_{\mathrm{ss}}\right|\left\|\tilde{\mathbf{p}}_{\mathrm{ss}}-\mathbf{p}_{\mathrm{ss}}\right\|_{1}\right]$ in the leading order.
Therefore, when metastable phases differ in observable averages [up to the correction in Eq. (8)], the long-time dynamics can be observed by measuring the observable average or autocorrelation. For example, for an observable chosen as a dual basis operator $O=\tilde{P}_{l}$ in Eq. (20), we simply have $\tilde{\mathbf{o}}^{T} \mathbf{p}(t)=[\mathbf{p}(t)]_{l}, l=1, \ldots, m$. Furthermore, when the approximation in Eq. (39) holds for times after the final relaxation [cf. Eq. (40)], the dynamics of averages and autocorrelations of all system observables is effectively classical [cf. Eq. (41)]. In particular, if the measurement of an observable $O$ is noninvasive, i.e., does not disrupt basins of attractions of metastable phases, $(\tilde{\mathbf{O}})_{k l} \approx \delta_{k l}(\tilde{\mathbf{o}})_{l}, k, l=$ $1, \ldots, m$, the long-time dynamics leads to the decay of the autocorrelations exactly as the decay of the autocorrelation of $\tilde{\mathbf{o}}$ in the classical dynamics: from the observable $\tilde{\mathbf{o}}$ variance in $\mathbf{p}_{\mathrm{ss}}$ during the metastable regime, toward 0 achieved after the final relaxation (cf. Ref. [94]). This is the case for the dual basis operators in Eq. (20), with the distance between the matrices bounded by $\lesssim 3\left(\mathcal{C}_{+}+\tilde{\mathcal{C}}_{\mathrm{cl}}+\mathcal{C}_{\mathrm{cl}} / 2\right)$ (see Sec. D1c in Ref. [55]). Finally, higher-order correlations, also between different observables, can be analogously approximated by correlations in classical dynamics.

\section{B. Classical characteristics of quantum trajectories}

In Sec. VA, we showed that the dynamics of the average system state can be approximated with the classical dynamics generated by a classical stochastic generator. Now we argue that this relation pertains to individual experimental realizations of system evolution [59]. Therefore, stochastic transitions between metastable phases can be observed in continuous measurement records or the system state sampled in QJMC simulations [8-12]—so called quantum trajectories (see Fig. 4). First, we show that statistics of quantum trajectories can be directly related to the statistics of classical stochastic trajectories. Second, we argue how coarse-graining in time returns classical trajectories between metastable phases, which for metastable phases differing in activity is the mechanism behind the phenomena of intermittence $[24,60]$ and dynamical heterogeneity [50,51], and leads to multimodal distribution of integrated measurement records during the metastable regime. Finally, we explain how system metastability can manifest itself as proximity to a first-order dynamical phase transition in the ensemble of quantum trajectories [60].

\section{Statistics of quantum trajectories}

Quantum trajectories describe the system state conditioned on a continuous measurement record, e.g., counting or homodyne measurement of photons emitted by the system due to action of jump operators in Eq. (1). In particular, the statistics of the total number of jumps in a quantum trajectory (total number of detected photons) is encoded by the biased or "tilted" master operator [24,60]

$$
\mathcal{L}_{s}(\rho)=\mathcal{L}(\rho)+\left(e^{-s}-1\right) \mathcal{J}(\rho),
$$

where $\mathcal{J}(\rho) \equiv \sum_{j} J_{j} \rho J_{j}^{\dagger}$. That is, $\Theta(s, t) \equiv \ln \left(\operatorname{Tr}\left\{e^{t \mathcal{L}_{s}}\right.\right.$ $[\rho(0)]\})$ is the cumulant generating function for the number $K(t)$ of jumps that occurred until time $t$ for quantum trajectories initialized in $\rho(0)$. The rates of the asymptotic 
(a)

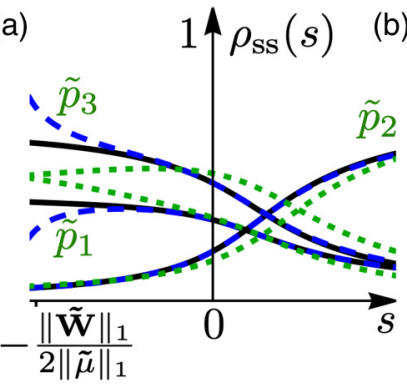

(e)

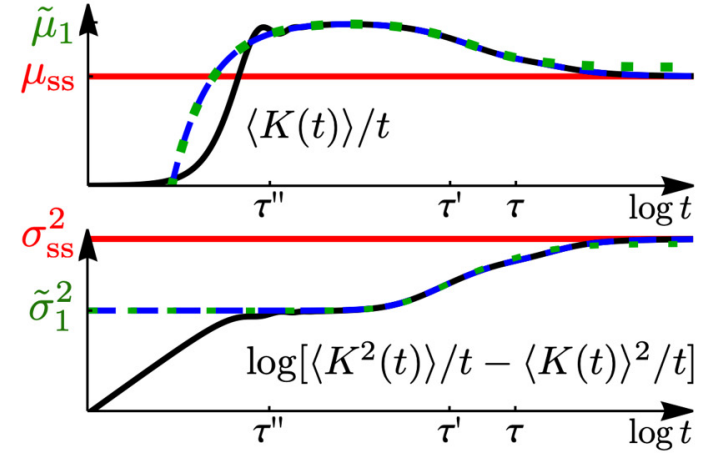

(c)

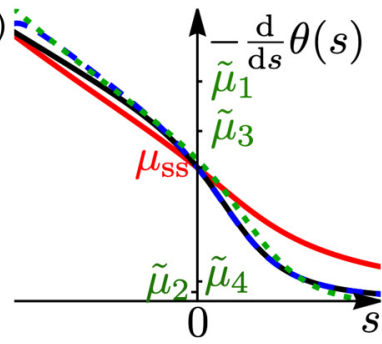

(d)

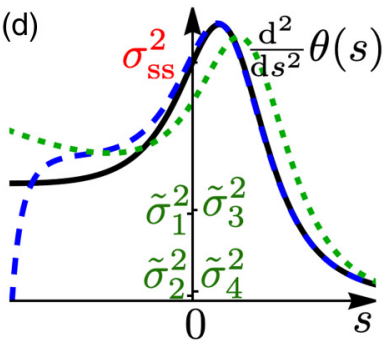

(g)

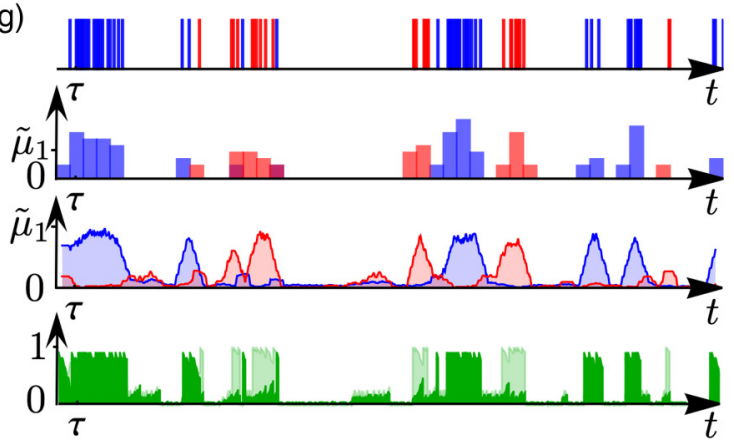

FIG. 4. Classical features of quantum trajectories: (a), (b) Approximating the biased operator of jump activity $\mathcal{L}_{s}$ [Eq. (44)] by the biased classical generator $\mathbf{W}_{s}$ [Eq. (47)] gives the approximations of: $\rho_{\mathrm{ss}}(s)$ (black solid) by the maximal eigenvector of $\mathbf{W}_{s}$ (green short-dashed) [panel (a); we plot $\operatorname{Tr}\left[\tilde{P}_{l} \rho_{\mathrm{ss}}(s)\right], l=1, \ldots, m$; cf. Eq. (20)], $\theta(s)$ by the cumulant generating function of total activity in classical trajectories [panel (b)], which are valid for $s$ in the perturbative regime of the statistics captured by $m=4$ slow modes (blue dashed) [Eq. (48)], as given by Eqs. (50) and (51). (c), (d) Similarly, $-d \theta(s) / d s=e^{-s} \sum_{j} \operatorname{Tr}\left[J_{j}^{\dagger} J_{j} \rho_{\mathrm{ss}}(s)\right]-\left(e^{-s}-1\right) \sum_{j} \operatorname{Tr}\left[J_{j}^{\dagger} J_{j} d \rho_{\mathrm{ss}}(s) / d s\right]$ (black solid), with the first term being the activity of $\rho_{\mathrm{ss}}(s)$ (red solid), is captured by the first derivative of the classical cumulant generating function (green short-dashed), up to non-Poissonian contribution to fluctuations in metastable phases [cf. Eq. (54)]. This contribution can be neglected for internal activity dominating classical dynamics, in which case the leading contribution to fluctuations is the result of long timescales of mixing between metastable phases rather than fluctuations within, as demonstrated in panel (d). (e), (f) Not only asymptotically, but already for times after the initial relaxation, the average rate and the fluctuation rate of jump number $K(t)$ (black solid) can be approximated by the constant contribution $\tilde{K}$ from before the metastable regime and the contribution from the dynamics within the MM (blue dashed), with the latter approximated by the corresponding rate for classical total activity $K_{\mathrm{cl}}(t)$ (green short-dashed) [cf. Eqs. (52) and (54)]. (g) Coarse-graining of jump records (top) in time gives values close to metastable phases activity (upper center) [cf. panel (c)], up to fluctuations which decrease with grain size (here $\delta t=0.7 \tau^{\prime}$; cf. Sec. V B 4). In turn, they capture the average activity of the conditional system state $|\psi(t)\rangle$ (lower center; we plot running average over $\delta t$, and the metastable phase support where $|\psi(t)\rangle$ is found [bottom; we plot $\left.\left\langle\psi(t)\left|\tilde{P}_{l}\right| \psi(t)\right\rangle, l=1,3\right]$.

statistics are determined then by $\theta(s) \equiv \lim _{t \rightarrow \infty} \Theta(s, t) / t$, which is simply the eigenvalue of $\mathcal{L}_{s}$ with the largest real part. We denote the associated (positive) eigenmatrix as $\rho_{\mathrm{ss}}(s)$ and choose the normalization $\operatorname{Tr}\left[\rho_{\mathrm{ss}}(s)\right]=1$, so that $\theta(s)=$ $\operatorname{Tr}\left\{\mathcal{L}_{s}\left[\rho_{\mathrm{ss}}(s)\right]\right\}=\left(e^{-s}-1\right) \sum_{j} \operatorname{Tr}\left[J_{j}^{\dagger} J_{j} \rho_{\mathrm{ss}}(s)\right]$. Then, $\rho_{\mathrm{ss}}(s)$ is the average asymptotic state of the system in trajectories with the probability biased by the factor $e^{-s K(t)}$, while the derivatives of $\theta(s)$ correspond to asymptotic rate of the corresponding cumulants. In particular, for a unique stationary state $\rho_{\mathrm{ss}}$ and the bias $|s|$ small enough with respect to the gap $-\operatorname{Re}\left(\lambda_{2}\right), \rho_{\mathrm{ss}}(s)=\rho_{\mathrm{ss}}+\cdots$, while

$$
\begin{gathered}
\theta(s)=\left(e^{-s}-1\right) \mu_{\mathrm{ss}}+\cdots, \\
k(s) \equiv-\frac{d}{d s} \theta(s)=e^{-s} \mu_{\mathrm{ss}}+\cdots
\end{gathered}
$$

where $\mu_{\mathrm{ss}} \equiv \sum_{j} \operatorname{Tr}\left[J_{j}^{\dagger} J_{j} \rho_{\mathrm{ss}}\right]$ (cf. Ref. [95]), so that the asymptotic jump rate - the asymptotic activity-is determined by the stationary state.

The nonanalyticities of $\theta(s)$ can be recognized as dynamical phase transitions [60], in analogy to nonanalyticities of the free energy in equilibrium statistical mechanics. In par- ticular, a first-order dynamical phase transition occurs at $s_{c}$ for which the maximal eigenvalue of $\mathcal{L}_{s_{c}}$ is not unique, so that the asymptotic activity $k(s)=-d \theta(s) / d s$ is no longer continuous, but features a jump at $s_{c}[24,51,60,64]$.

Similarly, statistics for integrated homodyne current and for time-integral of system observables are considered [96-101] (see also Secs. E3b and E3c in Ref. [55]).

\section{Classical tilted generator}

We now present our first result regarding classicality of quantum trajectories. We argue that the tilted master operator in Eq. (44) can be approximated by a tilted classical generator encoding the statistics in stochastic trajectories of the classical dynamics in Eq. (36). This leads to classical approximations for the asymptotic rate of the cumulant generating function and for the asymptotic system state in biased quantum trajectories.

The statistics of total activity [102-104] in classical dynamics is encoded by a biased or "tilted" classical generator [for reviews see Refs. [105,106]; cf. Eqs. (36) and (44)]

$$
\mathbf{W}_{s}=\mathbf{W}+\left(e^{-s}-1\right)\left(\mathbf{J}+\tilde{\boldsymbol{\mu}}^{\text {in }}\right),
$$


where $(\mathbf{J})_{k l} \equiv\left(1-\delta_{k l}\right)(\mathbf{W})_{k l}, k, l=1, \ldots, m$ encodes the transition rates in the classical dynamics, while $\left(\tilde{\boldsymbol{\mu}}^{\mathrm{in}}\right)_{k l} \equiv$ $\delta_{k l}\left[\tilde{\mu}_{l}+(\tilde{\mathbf{W}})_{l l}\right]$ with $\tilde{\mu}_{l} \equiv \sum_{j} \operatorname{Tr}\left(J_{j}^{\dagger} J_{j} \tilde{\rho}_{l}\right), k, l=1, \ldots, m$, encodes the average internal activity in metastable phases (which here is assumed Poissonian distributed; cf. Sec. E1 in Ref. [55]).

In Sec. E3d of Ref. [55], we show that the tilted classical generator $\mathbf{W}_{s}$ approximates, in the metastable phase basis, the tiled master operator $\mathcal{L}_{s}$ when the latter is restricted to the low-lying modes [cf. Eq. (34)],

$$
\left(\tilde{\mathbf{W}}_{s}\right)_{k l} \equiv \operatorname{Tr}\left[\tilde{P}_{k} \mathcal{L}_{s}\left(\tilde{\rho}_{l}\right)\right]
$$

$k, l=1, \ldots, m$, with the corrections bounded as [107]

$$
\begin{aligned}
\left\|\tilde{\mathbf{W}}_{s}-\mathbf{W}_{s}\right\| \lesssim & 2 e^{-s}\|\tilde{\mathbf{W}}\|_{1} \sqrt{\mathcal{C}_{\mathrm{cl}}}+\left|e^{-s}-1\right| m \\
& \times\left\|H+\frac{i}{2} \sum_{j} J_{j}^{\dagger} J_{j}\right\|_{\max } \sqrt{2 \mathcal{C}_{\mathrm{cl}}+4 \mathcal{C}_{+}} .
\end{aligned}
$$

For dynamics of classical systems with metastability, or, more generally, for the basis of metastable phases in Eq. (17) commuting with the dual basis in Eq. (20), $m \sqrt{2 \mathcal{C}_{\mathrm{cl}}+4 \mathcal{C}_{+}}$in Eq. (49) can be reduced to $2\left(\tilde{\mathcal{C}}_{\mathrm{cl}}+\mathcal{C}_{+}\right)$.

Since the biased dynamics $\mathcal{L}_{s}$ in Eq. (44) can be considered as the perturbation of the master operator $\mathcal{L}$ in Eq. (1) with $\left(e^{-s}-1\right) \mathcal{J}$, for bias $|s|$ much smaller than the separation to the fast eigenmodes, $\lambda_{m}^{R}-\lambda_{m+1}^{R}$, the $m$ low-lying eigenmodes and eigenvalues of $\mathcal{L}_{S}$ in Eq. (44) are approximated by those of $\mathcal{P} \mathcal{L}_{S} \mathcal{P}$ [95] or, in the metastable phase basis, those of $\tilde{\mathbf{W}}_{S}$ in Eq. (48). From Eq. (49), it then follows that the average asymptotic state in biased quantum trajectories is approximated by the asymptotic probability distribution in biased classical trajectories,

$$
\rho_{\mathrm{ss}}(s)=\sum_{l=1}^{m}\left[\mathbf{p}_{\mathrm{ss}}(s)\right]_{l} \tilde{\rho}_{l}+\cdots,
$$

where $\mathbf{p}_{\mathrm{ss}}(s)$ is the maximal eigenmode of $\mathbf{W}_{s}$ [see Fig. 4(a)], while the asymptotic rate of the cumulant generating function is approximated via the asymptotic total activity in biased classical trajectories,

$$
\theta(s)=\left(e^{-s}-1\right) \sum_{l=1}^{m}\left[\mathbf{p}_{\mathrm{ss}}(s)\right]_{l} \tilde{\mu}_{l}^{\mathrm{tot}}+\cdots,
$$

where $\tilde{\mu}_{l}^{\text {tot }} \equiv \sum_{k=1}^{m}(\mathbf{J})_{k l}+\tilde{\mu}_{l}^{\text {in }}, l=1, \ldots, m$, are the average total activities in the metastable phases (cf. Sec. E3f in Ref. [55]), so that the right-hand side of Eq. (51) is the maximal eigenvalue of $\mathbf{W}_{s}$ [see Fig. 4(b)]. For corrections from non-Hermitian perturbation theory [95], see Sec. E3a in Ref. [55]. One can consider similarly approximating $d \theta(s) / d s$ and $d^{2} \theta(s) / d s^{2}$, but additional contributions arise from non-Poissonian fluctuations in metastable phases (see Sec. V B 3 and Sec. E3a in Ref. [55]). Nevertheless, those can be neglected when the internal activities of metastable phases dominate transition rates of classical dynamics [see Figs. 4(c) and 4(d)], making Eq. (49) the crucial result in the link between the system metastability and its proximity to a first-order dynamical phase transition, which we discuss in Sec. V B 5.
In Secs. E3b and E3c of Ref. [55], we show that in the presence of classical metastability generators of statistics of integrated homodyne current and time-integral of system observables can be similarly linked to generators of statistics in classical trajectories, but with respect to time-integrals of their average value in metastable phases.

\section{Classical cumulants}

We now discuss how the dynamics of the first and the second cumulants of the jump number, directly accessible in experiments via counting measurement, are governed by the classical long-time dynamics for times after the initial relaxation. In particular, we argue how the asymptotic activity and fluctuation rate are approximated by the total activity and total fluctuation rates in the classical dynamics. These results establish a further correspondence between statistics of quantum and classical trajectories for times during and after the metastable regime, and, even asymptotically, they do not directly follow from Eq. (51) as cumulants are encoded by derivatives of the rate function [cf. Figs. 4(c) and 4(d)].

Classical dynamics of first cumulant. For times $t \geqslant t^{\prime \prime}$ such that $t\|\tilde{\mathbf{W}}\|_{1} \ll 1 / \sqrt{\mathcal{C}_{\mathrm{cl}}}$, the rate of average jump number is approximated by the time-integral of the total activity in classical trajectories, $K_{\mathrm{cl}}(t)$, whose statistics in encoded by $\mathbf{W}_{s}$ of Eq. (47), together with the constant contribution $\tilde{K}$ to the jump number accumulated before the metastable regime (cf. Fig. 4(e) and see Sec. E3e of Ref. [55]),

$$
\begin{aligned}
\frac{\langle K(t)\rangle}{t} & =\frac{\left\langle K_{\mathrm{cl}}(t)\right\rangle}{t}+\frac{\tilde{K}}{t}+\cdots, \\
& \equiv \frac{1}{t} \int_{0}^{t} d t_{1} \sum_{l=1}^{m}\left[\tilde{\boldsymbol{\mu}}^{\mathrm{tot}} \mathbf{p}\left(t_{1}\right)\right]_{l}-\frac{\operatorname{Tr}\{\mathcal{J} \mathcal{S} \mathcal{Q}[\rho(0)]\}}{t}+\cdots,
\end{aligned}
$$

where $\left(\tilde{\boldsymbol{\mu}}^{\mathrm{tot}}\right)_{k l} \equiv \delta_{k l} \tilde{\mu}_{l}^{\text {tot }}, \mathbf{p}(t)=e^{t \mathbf{W}} \tilde{\mathbf{p}}, \mathcal{S}$ is the pseudoinverse of the master operator $\mathcal{L}$ in Eq. (1), $\mathcal{Q} \equiv \mathcal{I}-\mathcal{P}$ is the projection on the fast-modes of the dynamics [cf. Eq. (4)]. Therefore, similarly as for averages of system observables [Eq. (42)], the classical dynamics can be observed by measuring $\langle K(t)\rangle$ when the metastable phases differ in the total activity.

When the approximation in Eq. (52) holds for time $t$ after the final relaxation [cf. Fig. 3(c)], the asymptotic activity in quantum trajectories is approximated by the asymptotic total activity of classical trajectories [cf. Fig. 4(e)]

$$
\begin{aligned}
\mu_{\mathrm{ss}} & \equiv \lim _{t \rightarrow \infty} \frac{\langle K(t)\rangle}{t}=\sum_{j} \operatorname{Tr}\left(J_{j}^{\dagger} J_{j} \rho_{\mathrm{ss}}\right) \\
& =\lim _{t \rightarrow \infty} \frac{\left\langle K_{\mathrm{cl}}(t)\right\rangle}{t}+\cdots=\sum_{l=1}^{m}\left(\tilde{\boldsymbol{\mu}}^{\mathrm{tot}} \mathbf{p}_{\mathrm{ss}}\right)_{l}+\cdots,
\end{aligned}
$$

where $\mathbf{p}_{\mathrm{ss}}$ is the stationary distribution of the classical dynamics $\mathbf{W}$. The corrections are bounded by $\lesssim \max _{1 \leqslant l \leqslant m}\left|\tilde{\mu}_{l}\right|\left\|\tilde{\mathbf{p}}_{\mathrm{ss}}-\mathbf{p}_{\mathrm{ss}}\right\|_{1}+\|\tilde{\mathbf{W}}\| \sqrt{\mathcal{C}_{\mathrm{cl}}} \quad\left[\right.$ as $\operatorname{Tr}\left[\mathcal{J}\left(\rho_{\mathrm{ss}}\right)\right]=$ $\tilde{\sum}_{l=1}^{m}\left(\tilde{\boldsymbol{\mu}} \tilde{\mathbf{p}}_{\mathrm{ss}}\right)_{l}$; cf. Eqs. (41) and (37)].

Classical dynamics of second cumulant. For times such that $\|\mathcal{S} \mathcal{Q}\|\|\mathcal{J}\| \ll t\|\tilde{\boldsymbol{\mu}}\|_{1}$ and $t\|\tilde{\mathbf{W}}\|_{1} \ll$ $\min \left(1 / \sqrt{\mathcal{C}_{\mathrm{cl}}}, \sqrt{\|\tilde{\mathbf{R}}\|_{1}\|\tilde{\mathbf{W}}\|_{1} / \sqrt{\mathcal{C}_{\mathrm{cl}}}}\right)$, where $\tilde{\mathbf{R}}$ denotes the 
pseudoinverse of the long-time-dynamics generator $\tilde{\mathbf{W}}$ in Eq. (34), the rate of fluctuations of jump number is approximated by the rate of fluctuations of total activity in classical trajectories, corrected by non-Poissonian fluctuations in metastable phases and by the contribution to the average from before the metastable regime (see Fig. 4(f) and Sec. E3e in Ref. [55])

$$
\begin{aligned}
& \frac{\left\langle K^{2}(t)\right\rangle-\langle K(t)\rangle^{2}}{t} \\
& =\frac{\left\langle K_{\mathrm{cl}}^{2}(t)\right\rangle-\left\langle K_{\mathrm{cl}}(t)\right\rangle^{2}}{t}+\frac{\left\langle\Delta_{\mathrm{cl}}(t)\right\rangle}{t} \\
& \quad-\sum_{k, l=1}^{m} \tilde{p}_{k} \tilde{p}_{l} \frac{\left\langle K_{\mathrm{cl}}^{(k)}(t)\right\rangle-\left\langle K_{\mathrm{cl}}^{(l)}(t)\right\rangle}{t}\left(\tilde{K}_{k}-\tilde{K}_{l}\right)+\cdots,
\end{aligned}
$$

where

$$
\begin{aligned}
\left\langle K_{\mathrm{cl}}^{2}(t)\right\rangle= & \int_{0}^{t} d t_{1} \sum_{l=1}^{m}\left[\tilde{\boldsymbol{\mu}}^{\mathrm{tot}} \mathbf{p}\left(t_{1}\right)\right]_{l}+2 \int_{0}^{t} d t_{1} \int_{0}^{t-t_{1}} d t_{2} \\
& \times \sum_{l=1}^{m}\left[\left(\mathbf{J}+\tilde{\boldsymbol{\mu}}^{\mathrm{in}}\right) e^{t_{2} \mathbf{W}}\left(\mathbf{J}+\tilde{\boldsymbol{\mu}}^{\mathrm{in}}\right) \mathbf{p}\left(t_{1}\right)\right]_{l},
\end{aligned}
$$

and we denoted

$$
\left\langle\Delta_{\mathrm{cl}}(t)\right\rangle \equiv \int_{0}^{t} d t_{1} \sum_{l=1}^{m}\left[\boldsymbol{\delta}_{\tilde{\sigma}^{2}} \mathbf{p}\left(t_{1}\right)\right]_{l},
$$

with $\left(\delta_{\tilde{\sigma}^{2}}\right)_{k l} \equiv-\delta_{k l} 2 \operatorname{Tr}\left[\mathcal{J S} \mathcal{Q} \mathcal{J}\left(\tilde{\rho}_{l}\right)\right], k, l=1, \ldots, m$, so that $\left(\tilde{\sigma}^{\text {tot }}\right)^{2} \equiv \tilde{\mu}^{\text {tot }}+\delta_{\tilde{\sigma}^{2}}$ are rates of total fluctuations in metastable phases (see Sec. E3f in Ref. [55]), $\left\langle K_{\mathrm{cl}}^{(l)}(t)\right\rangle$ is the average of $K_{\mathrm{cl}}(t)$ for $l$ th metastable phase in Eq. $(52)$, i.e., $(\tilde{\mathbf{p}})_{k}=\delta_{k l}$, and $\tilde{K}_{l} \equiv \operatorname{Tr}\left\{\tilde{P}_{l} \mathcal{J S} \mathcal{S}[\rho(0)]\right\} / \operatorname{Tr}\left[\tilde{P}_{l} \rho(0)\right]$ is the contribution to the jump number from before the metastable regime conditioned on the metastable phase that the system evolves into. Therefore, similarly as for autocorrelations of system observables [Eq. (43)], the classical dynamics can be observed even for the stationary state, by measuring fluctuations of $K(t)$ whenever the metastable phases differ in the total activity.

When the approximation in Eq. (54) is valid for times after the final relaxation, the asymptotic fluctuation rate $[40,108]$

$$
\begin{aligned}
\sigma_{\mathrm{ss}}^{2} & \equiv \lim _{t \rightarrow \infty} \frac{\left\langle K^{2}(t)\right\rangle-\langle K(t)\rangle^{2}}{t} \\
& =\sum_{j} \operatorname{Tr}\left(J_{j}^{\dagger} J_{j} \rho_{\mathrm{ss}}\right)-2 \operatorname{Tr}\left[\mathcal{J} \mathcal{S} \mathcal{J}\left(\rho_{\mathrm{ss}}\right)\right]
\end{aligned}
$$

is approximated by the asymptotic rate of fluctuations of total activity in classical trajectories, corrected by non-Poissonian contribution to fluctuations in metastable phases (cf. Fig. 4(f) and see Secs. B1 and E3g in Ref. [55])

$$
\begin{aligned}
\sigma_{\mathrm{ss}}^{2} & =\lim _{t \rightarrow \infty}\left[\frac{\left\langle K_{\mathrm{cl}}^{2}(t)\right\rangle-\left\langle K_{\mathrm{cl}}(t)\right\rangle^{2}}{t}+\frac{\left\langle\Delta_{\mathrm{cl}}(t)\right\rangle}{t}\right]+\cdots \\
& =\sum_{l=1}^{m}\left\{\left[\tilde{\boldsymbol{\mu}}^{\mathrm{tot}}-2\left(\mathbf{J}+\tilde{\boldsymbol{\mu}}^{\mathrm{in}}\right) \mathbf{R}\left(\mathbf{J}+\tilde{\boldsymbol{\mu}}^{\mathrm{in}}\right)+\boldsymbol{\delta}_{\tilde{\boldsymbol{\sigma}}^{2}}\right] \mathbf{p}_{\mathrm{ss}}\right\}_{l}+\cdots \\
& =\sum_{l=1}^{m}\left[\left(\tilde{\boldsymbol{\mu}}^{\mathrm{tot}}-2 \tilde{\boldsymbol{\mu}}^{\mathrm{tot}} \mathbf{R} \tilde{\boldsymbol{\mu}}^{\mathrm{tot}}+\boldsymbol{\delta}_{\tilde{\boldsymbol{\sigma}}^{2}}\right) \mathbf{p}_{\mathrm{ss}}\right]_{l}+\cdots
\end{aligned}
$$

where $\mathbf{R}$ denotes the pseudoinverse of the classical stochastic generator $\mathbf{W}$ in Eq. (36) and the last equality follows by noting that $\mathbf{J}+\tilde{\boldsymbol{\mu}}^{\text {in }}=\mathbf{W}+\tilde{\boldsymbol{\mu}}^{\text {tot }}$ and thus $\left(\mathbf{J}+\tilde{\boldsymbol{\mu}}^{\mathrm{in}}\right) \mathbf{p}_{\mathrm{ss}}=\tilde{\boldsymbol{\mu}}^{\text {tot }} \mathbf{p}_{\mathrm{ss}}$.

Other statistics. Similarly to Eqs. (52), (54), (53), and (58), the first and the second cumulants for integrated homodyne current or for time-integrals of system observables can be related to the statistics in classical dynamics with respect to observables given by the corresponding averages for metastable phases (see Secs. E3b and E3c in Ref. [55]). Furthermore, the integrals of average and autocorrelations of system measurements in Eqs. (42) and (43) can be approximated analogously.

\section{Classical dynamics of quantum trajectories}

For systems exhibiting metastability in the system dynamics, individual evolutions of the system over time typically exhibit intermittence (distinct periods of jump activity isolated in time) or dynamical heterogeneity (distinct periods of jump activity isolated both in time and space) in the emission measurement record or time-integral of observables [see Fig. 1(e)]. We now explain that these features can be understood in terms of classical dynamics between the metastable phases whose differences in internal (global or local) jump activity dominate transition rates of that dynamics (see also Refs. [41,109]). To this aim, using results of Sec. V B 3, we establish a direct relation between classical trajectories and time-coarse-grained records of continuous measurements. Therefore, we prove that metastability can be observed not only on average [cf. Eqs. (42) and (43)], but also in individual realizations of continuous measurement experiments and individual samples of QJMC simulations provided that the metastable regime is long enough.

As a corollary of our results, integrated continuous measurements can be used to distinguish metastable phases during the metastable regime, as their distribution is multimodal with distinct modes corresponding to the metastable phases differing in the rate of the measurement average. Interestingly, during the metastable regime all continuous measurements lead to negligible disturbance of the system as on average they simply correspond to the system dynamics, so that the disturbance is bounded by $2 \mathcal{C}_{\mathrm{MM}}$ [cf. Eq. (10) and Sec. IV B].

Time-coarse-grained measurement records as classical trajectories. We focus here on the measurement of total number of jumps that occur in the system, but analogous arguments hold for the measurements of local jump activity (see Sec. E3a in Ref. [55]) and of homodyne current (cf. Sec. E3b in Ref. [55]).

Consider course-graining in time of a record of jump counting measurement, with the activity in time bins $\delta t$,

$$
k(n) \equiv \frac{K[(n+1) \delta t]-K(n \delta t)}{\delta t},
$$

for $n=0,1,2, \ldots$. We argue that time-coarse-grained measurement records can be interpreted as classical trajectories between metastable phases when the internal activity dominates the long time dynamics, $\|\tilde{\boldsymbol{\mu}}\|_{1} \gg\|\tilde{\mathbf{W}}\|_{1}$, and $\delta t$ is chosen long enough within the metastable regime, as in this case the activity typically attains only values of the internal activities in metastable phases [see Fig. 4(g)]. 
From Eq. (52), for $\delta t \leqslant t^{\prime}$ such that $\|\mathcal{S} \mathcal{Q}\|\|\mathcal{J}\| \ll \delta t\|\tilde{\boldsymbol{\mu}}\|_{1}$ and $t\|\tilde{\mathbf{W}}\|_{1} \ll 1 / \sqrt{\mathcal{C}_{\mathrm{cl}}}$, the average activity in trajectories originating in $\rho_{\text {cond }}(t)$ at time $t=n \delta t$ is approximated as

$$
\langle k(n)\rangle_{\rho_{\text {cond }}(t)}=\sum_{l=1}^{m} \tilde{\mu}_{l}^{\text {in }} \tilde{p}_{l}(n)+\cdots,
$$

where $\tilde{p}_{l}(n) \equiv \operatorname{Tr}\left[\tilde{P}_{l} \rho_{\text {cond }}(t)\right]$ determines the metastable state. Similarly, from Eq. (54), the variance

$$
\begin{aligned}
& \left\langle k^{2}(n)\right\rangle_{\rho_{\text {cond }}(t)}-\langle k(n)\rangle_{\rho_{\text {cond }}(t)}^{2} \\
& =\sum_{l=1}^{m} \tilde{p}_{l}(n) \frac{\left(\tilde{\sigma}_{l}^{\text {tot }}\right)^{2}}{\delta t} \\
& \quad+\sum_{k, l=1}^{m} \tilde{p}_{k}(n) \tilde{p}_{l}(n) \frac{\left(\tilde{\mu}_{k}^{\text {in }}-\tilde{\mu}_{l}^{\text {in }}\right)^{2}}{2} \\
& \quad+\sum_{k, l=1}^{m} \tilde{p}_{k}(n) \tilde{p}_{l}(n) \frac{\left(\tilde{\mu}_{k}^{\text {in }}-\tilde{\mu}_{l}^{\text {in }}\right)\left(\tilde{K}_{k}-\tilde{K}_{l}\right)}{\delta t}+\cdots
\end{aligned}
$$

(by assuming $\left(1+\tilde{\mathcal{C}}_{\mathrm{cl}} / 2\right) \mathcal{C}_{\mathrm{MM}} \ll 1$; see Sec. E3f in Ref. [55]).

When the conditional state evolves into a single metastable phase $\mathcal{P}\left[\rho_{\text {cond }}(t)\right]=\tilde{\rho}_{l}$, the average activity is approximated by the activity $\tilde{\mu}_{l}^{\text {in }}$ of $l$ th metastable phase [cf. Fig. 4(e)] and its variance $\left(\tilde{\sigma}_{l}^{\text {tot }}\right)^{2} / \delta t$ decays inversely with the increasing time-bin length $\delta t$ [cf. Fig. 4(f)]. Therefore, for long enough metastable regime, $\delta t$ can be chosen so that the fluctuations between measurement records become negligible, and the activity typically takes values approximately equal the average $\tilde{\mu}_{l}^{\text {in }}$ [cf. Fig. $4(\mathrm{~g})$ (center)]. For $\rho_{\text {cond }}(t)$ evolving into a mixture of metastable phases, however, a constant term is present in Eq. (61) because of a multimodal distribution of the activity number in $n$th time bin. Namely, when $\tilde{\mathcal{C}}_{\mathrm{cl}} \ll$ 1 , the distribution can be approximated, up to corrections $2\left(\mathcal{C}_{\mathrm{MM}}+\tilde{\mathcal{C}}_{\mathrm{cl}}\right)+\mathcal{C}_{\mathrm{cl}}$, as a mixture, with probabilities approximating $\tilde{p}_{l}(n)$, of distributions with averages equal internal activities of metastable phase, $\tilde{\mu}_{l}^{\text {in }}$, and variances inversely proportional to $\delta t, l=1, \ldots, m$.

This is proved in Sec. E3h of Ref. [55], by postselecting trajectories in terms of probability of the final state in the time bin, $\rho_{\text {cond }}(t+\delta t)$, evolving (on average) into a metastable phase $\tilde{\rho}_{l}$, which, formally, corresponds to performing at $t+\delta t$ the measurement in Eq. (26) that approximates $\tilde{P}_{l}$ in Eq. (20) [cf. Fig. 4(g) (bottom)].

We conclude that, for long enough $\delta t$, the activity $k(n)$, $n=0,1,2, \ldots$, takes in typical measurement records only $m$ values $\tilde{\mu}_{l}^{\text {in }}, l=1, \ldots, m$, corresponding to the internal activities of $m$ metastable phases (approximately, up to fluctuations decaying inversely in $\delta t$ ). For the bimodal case $m=2$, see also Ref. [41].

Dynamics of time-coarse-grained measurement records as classical long-time dynamics. We now argue that transitions in coarse-grained measurement records are captured by the generator of the effective long-time dynamics $\mathbf{W}$ [Eq. (36)]. In particular, the effective lifetime of the $l$ th metastable phase in coarse-grained trajectories is approximated by $\tau_{l} \equiv$ $-1 /(\mathbf{W})_{l l}, l=1, \ldots, m$.

From the discussion above, for an initial state $\rho(0)$, the distribution of activity $k(0)$ can be approximated, up to small fluctuations, by a probability distribution over metastable phase activities $\tilde{\mu}_{l}^{\text {in }}$, with probabilities approximated by $\tilde{p}_{l}=$ $\operatorname{Tr}\left[\tilde{P}_{l} \rho(0)\right], l=1, \ldots, m$. Analogously, the distribution of the activity $k(n)$ in a later $n$th time bin is approximated by $\tilde{p}_{k}(n)=$ $\left[\left(e^{\delta t \tilde{\mathbf{w}}}\right)^{n} \tilde{\mathbf{p}}\right]_{k}$, where $t=n \delta t$, which is further approximated by $\left[\left(e^{\delta t \mathbf{W}}\right)^{n} \tilde{\mathbf{p}}\right]_{k}, k=1, \ldots, m$ (cf. Eq. (39); corrections can be further reduced to $n \mathcal{C}_{\mathrm{cl}}$ by considering discrete stochastic dynamics; see Sec. E2f in Ref. [55]). Therefore, the transition matrix, i.e., the probability of observing $k(n) \approx \tilde{\mu}_{k}^{\text {in }}$ conditioned on the observation of the initial activity $k(0) \approx \tilde{\mu}_{l}^{\text {in }}$, is approximated by the classical dynamics transition matrix $\left(e^{\delta t \mathbf{W}}\right)_{k l}$ (or a discrete stochastic dynamics; see Sec. E2f in Ref. [55]). This relation is further corroborated by Eqs. (52) and (54), with the average and variance of the integrated activity, $\sum_{n=0}^{\lfloor t / \delta t\rfloor} k(n)=K(\lfloor t / \delta t\rfloor \delta t) / \delta t$, approximately governed by the classical long-time dynamics $\mathbf{W}$ [cf. Figs. 4(e) and 4(f)].

\section{Classical metastability and dynamical phase transitions}

Finally, we explain how classical metastability can manifest itself as proximity to a first-order dynamical phase transition in the ensemble of quantum trajectories [60], i.e., to a first-order nonanalyticity of $\theta(s)$.

Metastable phases as eigenmodes of tilted generator. Building on the results of Sec. VB2, when the differences in the activity of the metastable phases dominate the transition rates of the classical dynamics between them, we can approximate $\tilde{\mathbf{W}}_{s}$ in Eq. (48) as

$$
\tilde{\mathbf{W}}_{s}=\mathbf{W}-h_{s} \tilde{\boldsymbol{\mu}}^{\mathrm{in}}+\cdots \equiv \mathbf{W}_{h_{s}}+\cdots,
$$

where $h_{s} \equiv 1-e^{-s}$ and $\mathbf{W}_{h_{s}}$ encodes the statistics of the timeintegral of the observable $\tilde{\boldsymbol{\mu}}^{\text {in }}$ in classical trajectories, rather than their activity (cf. Sec. E1 in Ref. [55]). The corrections in Eq. (62) additional to Eq. (49) are $\lesssim h_{s}\|\tilde{\mathbf{W}}\|_{1} / 2$ [replacing $\tilde{\boldsymbol{\mu}}^{\text {in }}$ by $\tilde{\boldsymbol{\mu}}$ or $\tilde{\boldsymbol{\mu}}^{\text {tot }}$ doubles them]. Furthermore, for the bias large enough, so that $h_{s}$ is finite, the contribution from $\mathbf{W}$ in $\mathbf{W}_{h_{s}}$ of Eq. (62) can be neglected. In that case, if the bias is still negligible with respect to the gap to the fast eigenmodes, $\lambda_{m}^{R}-\lambda_{m+1}^{R}, m$ low-lying eigenmodes of $\mathcal{L}_{s}$ are simply approximated by the metastable phases and the corresponding eigenvalues and their derivatives approximated analogously to Eqs. (45) and (46). In particular, the maximal eigenmode corresponds to the metastable phase with the maximum (for $s<0$ ) or minimum activity (for $s>0$ ), and

$$
\begin{gathered}
\theta(s)=\left(e^{-s}-1\right) \tilde{\mu}_{l(s)}+\cdots, \\
k(s)=e^{-s} \tilde{\mu}_{l(s)}+\cdots,
\end{gathered}
$$

where

$$
\tilde{\mu}_{l(s)} \equiv \begin{cases}\max _{1 \leqslant l \leqslant m} \tilde{\mu}_{l}^{\text {in }}, & s<0, \\ \min _{1 \leqslant l \leqslant m} \tilde{\mu}_{l}^{\text {in }}, & s>0 .\end{cases}
$$

(cf. Figs. 4(a)-4(c), and see Sec. E3a in Ref. [55] for corrections from non-Hermitian perturbation theory [95]). This is a key observation for the numerical method we introduce in Sec. VII B to find metastable phases as well as for the relation of metastability to dynamical phase transitions we explain next. 
Metastability as proximity to first-order dynamical phase transitions. For metastable phases differing in activity (or observable averages or homodyne current), Eq. (64) implies a sharp change in the derivative of $\theta(s)$, i.e., $-k(s)$, close to $s=0$ [see Fig. 4(c)]. This sharp change can be interpreted as the proximity to a first-order dynamical phase transition $[24,51,60,64]$. An analogous argument was made for the classical Markovian dynamics in Ref. [39].

A sharp change in $k(s)$ around $s=0$, implies in turn a large second derivative of $\theta(s)$ [see Fig. 4(d)]. In particular, $d^{2} \theta(s) / d s^{2}$ at $s=0$ determines the rate of fluctuations in jump number, which can be approximated as [cf. Eqs. (58) and (62)]

$$
\sigma_{\mathrm{ss}}^{2}=\sum_{l=1}^{m}\left[\left(\tilde{\boldsymbol{\sigma}}^{\mathrm{in}}\right)^{2} \mathbf{p}_{\mathrm{ss}}-2 \tilde{\boldsymbol{\mu}}^{\mathrm{in}} \mathbf{R} \tilde{\boldsymbol{\mu}}^{\mathrm{in}} \mathbf{p}_{\mathrm{ss}}\right]_{l}+\cdots,
$$

where $\left(\tilde{\boldsymbol{\sigma}}^{\text {in }}\right)^{2} \equiv \tilde{\boldsymbol{\mu}}^{\text {in }}+\boldsymbol{\delta}_{\tilde{\sigma}^{2}}$ and the additional corrections are bounded by $\|\tilde{\mathbf{W}}\|_{1}\left(1+2 \max _{1 \leqslant l \leqslant m}\left|\tilde{\mu}_{l}\right|\|\tilde{\mathbf{R}}\|_{1}\right)$. The fluctuation rate is indeed large for the stationary state being a mixture of metastable phases with different activities [cf. Figs. 4(a) and 4(d)]. This is a consequence of long timescales of the effective classical dynamics between metastable phases which govern the intermittence in emission records $[24,109]$ and are captured by the resolvent in the second term of Eq. (66). In contrast, when the stationary state corresponds to a single metastable phase (so that $\mathbf{R} \tilde{\boldsymbol{\mu}}{ }^{\text {in }} \mathbf{p}_{\mathrm{ss}} \propto \mathbf{R} \mathbf{p}_{\mathrm{ss}}=0$ ), the fluctuation rate is finite as fluctuations originate inside that metastable phase alone [up to corrections of Eq. (66)]. A large second derivative of $\theta(s)$ occurs then away from $s=0$ at intermediate (negative or positive) $s$ values.

In terms of phase-transition phenomenology, the proximity of a first-order dynamical phase transition manifests itself in a multimodal distribution of a dynamical quantity (i.e., the jump number) in (biased) trajectories for times within the metastability regime, while at longer times in the coexistence, within individual trajectories, of active and inactive regimes that can be considered as dynamical phases [cf. Fig. 4(g)]. These dynamical phases correspond directly to metastable phases (cf. Sec. V B 4).

Other statistics. Similar results to Eqs. (63) and (64), and thus the relation of metastability to dynamical phase transitions, also follow for: individual jump activity (see Sec. E 3a in Ref. [55]), integrated homodyne current (see Sec. E3b in Ref. [55] and cf. Ref. [100]) and time-integrals of system observables (see Sec. E3c in Ref. [55] and cf. Ref. [101]).

\section{CLASSICAL WEAK SYMMETRIES}

Here, we discuss how weak symmetries of the dynamics, i.e., symmetries of the master operator in Eq. (1), are inherited by MMs and long-time dynamics. For classical metastability, we find that nontrivial symmetries are necessarily discrete as they correspond to classical symmetries, i.e., approximate permutations of metastable phases, which are inherited by the classical long-time dynamics. Since first-order dissipative phase transitions occurring in thermodynamic limit manifest themselves as metastability for finite system size, our results
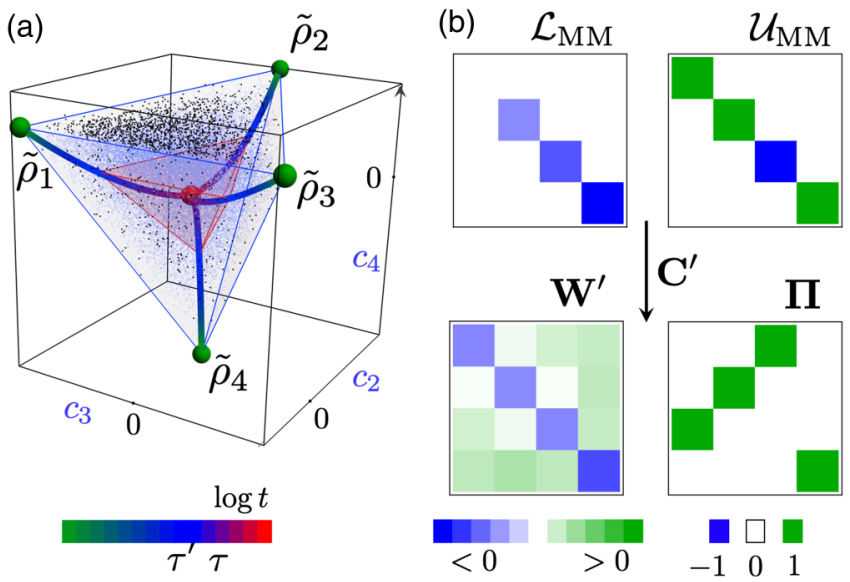

FIG. 5. Symmetry of dynamics and classical metastability: (a) A weak symmetry leads to the MM being symmetric under the corresponding transformation of coefficients, here $c_{3} \mapsto-c_{3}$ [cf. $\mathcal{U}_{\mathrm{MM}}$ in panel (b)], which is also preserved by the long-time dynamics (blue simplex at $t \ll \tau^{\prime}$, red simplex at $t=\tau^{\prime}$ ). States invariant under the symmetry, e.g., metastable phases $\tilde{\rho}_{2}$ and $\tilde{\rho}_{4}$ and the stationary state $\rho_{\mathrm{ss}}$ (red sphere), necessarily feature $c_{3}=0$. (b) The transformation $\mathbf{C}^{\prime}$ to the symmetric set of metastable phases in Eqs. (72) and (73) yields the classical long-time dynamics $\mathbf{W}^{\prime}$ symmetric with respect to the permutation $\Pi$ that corresponds to the action of the symmetry on metastable phases, and in this case swaps $\tilde{\rho}_{1}$ and $\tilde{\rho}_{3}$ [cf. Eqs. (69) and (77)].

pave a way for understanding symmetry breaking in open quantum systems (see also Ref. [42]).

By a weak symmetry we refer to the generator of the system dynamics $\mathcal{L}$ obeying a symmetry on the master operator level,

$$
[\mathcal{L}, \mathcal{U}]=0
$$

where $\mathcal{U}(\rho) \equiv U \rho U^{\dagger}$ with a unitary operator $U$ of a symmetry (see Refs. [61-63]). As we consider a unique stationary state, we are interested in the case when the symmetry operator $U$ is not itself conserved by the dynamics, so that in general $\mathcal{L}^{\dagger}(U) \neq 0$ (as the number of distinct stationary states is the same as the number of linearly independent conserved quantities [61,63]). For example, $\mathcal{U}$ can describe the translation symmetry in homogeneous dissipative systems with periodic boundary conditions.

From Eq. (67) it follows that $\mathcal{L}$ is block diagonal in the operator basis of eigenmatrices of $\mathcal{U}$. Therefore, the eigenmatrices of $\mathcal{L}, R_{k}$ (and $L_{k}$ of $\mathcal{L}^{\dagger}$ ) can be simultaneously chosen as eigenmatrices of $\mathcal{U}$ (and $\mathcal{U}^{\dagger}$ ), in which case $\mathcal{U}\left(R_{k}\right)=e^{i \delta \phi_{k}} R_{k}$ $\left[\mathcal{U}^{\dagger}\left(L_{k}\right)=e^{i \phi_{k}} L_{k}\right]$, where $\phi_{k}$ equals a difference in arguments of $U$ eigenvalues $(\bmod 2 \pi)$ (cf. Fig. 5 and see Sec. G1 in Ref. [55]).

\section{A. Symmetry and general metastability}

We first discuss how a weak symmetry in Eq. (67) affects the structure of a general MM and the long-time dynamics within it. 


\section{Symmetry of metastable manifolds}

As the set of all density matrices is invariant under any symmetry, its image under the dynamics featuring a weak symmetry is also symmetric at any time $t, \mathcal{U}\left\{e^{t \mathcal{L}}[\rho(0)]\right\}=$ $e^{t \mathcal{L}}\{\mathcal{U}[\rho(0)]\}$. In particular, a unique stationary state achieved asymptotically is necessarily symmetric $\mathcal{U}\left(\rho_{\mathrm{ss}}\right)=\rho_{\mathrm{ss}}$ (or, in the case of degeneracy, the manifold of stationary states is invariant). Similarly, the set of system states during the metastable regime, i.e., the set of metastable states, is invariant under the symmetry $\mathcal{U}$. This can be seen from the MM being determined by the projection $\mathcal{P}$ on the low-lying modes in Eq. (4), which in the presence of the weak symmetry fulfills

$$
[\mathcal{P}, \mathcal{U}]=0
$$

[cf. Eq. (67)]. This is a direct consequence of the modes of $\mathcal{L}$ being eigenmatrices of the symmetry, so that the coefficients gain a phase under the symmetry, $\operatorname{Tr}\left\{L_{k} \mathcal{U}[\rho(0)]\right\}=\operatorname{Tr}\left[\mathcal{U}^{\dagger}\left(L_{k}\right) \rho(0)\right]=e^{i \phi_{k}} \operatorname{Tr}\left[L_{k} \rho(0)\right]=e^{i \phi_{k}} c_{k}$, and thus $\mathcal{P}\{\mathcal{U}[\rho(0)]\}=\sum_{k=1}^{m} e^{i \phi_{k}} \operatorname{Tr}\left[L_{k} \rho(0)\right] R_{k}=$ $\sum_{k=1}^{m} \operatorname{Tr}\left[L_{k} \rho(0)\right] \mathcal{U}\left(R_{k}\right)=\mathcal{U}\{\mathcal{P}[\rho(0)]\}$; see Fig. 5(a).

\section{Symmetry of long-time dynamics}

The weak symmetry in Eq. (67) together with the symmetry of the MM in Eq. (68) yields the symmetry of the long-time dynamics in the MM in Eq. (6) as

$$
\left[\mathcal{L}_{\mathrm{MM}}, \mathcal{U}\right]=0=\left[\mathcal{L}_{\mathrm{MM}}, \mathcal{U}_{\mathrm{MM}}\right],
$$

where $\mathcal{U}_{\mathrm{MM}} \equiv \mathcal{P U} \mathcal{P} ; \quad$ see Fig. $5(\mathrm{~b})$. This follows since $[\mathcal{P} \mathcal{L} \mathcal{P}, \mathcal{U}]=\mathcal{P}[\mathcal{L}, \mathcal{U}] \mathcal{P}=0 \quad$ and $[\mathcal{P} \mathcal{L P}, \mathcal{P U} \mathcal{P}]=$ $\mathcal{P}[\mathcal{L}, \mathcal{U}] \mathcal{P}=0$ from Eq. $(68)$ and $[\mathcal{L}, \mathcal{P}]=0$.

\section{B. Symmetry and classical metastability}

We now explain how weak symmetries for classical metastability necessarily correspond to approximate permutations of metastable phases and thus any nontrivial continuous weak symmetries of low-lying modes preclude classical metastability. We also show the set of metastable phases can be chosen invariant under the symmetry, in which case, the sets of supports and basins of attractions of metastable phases are also invariant. Furthermore, both the long-time dynamics and its classical approximation are then symmetric with respect to the corresponding permutation. This restricts the structure of the low-lying eigenmodes, including the stationary state, and, in turn, simplifies the test of classicality introduced in Sec. III.

\section{Approximate symmetry of metastable phases}

The symmetry $\mathcal{U}$ in Eq. (67) transforms the projections $\tilde{\rho}_{1}, \ldots, \tilde{\rho}_{m}$ in Eq. (17) into $\mathcal{U}\left(\tilde{\rho}_{1}\right), \ldots, \mathcal{U}\left(\tilde{\rho}_{1}\right)$, which are also projections of system states [e.g., $\mathcal{U}\left(\rho_{1}\right), \ldots, \mathcal{U}\left(\rho_{m}\right)$ for states in Eq. (14)]. In the space of coefficients, the symmetry transformation is unitary, and does not change distances. Therefore, as the simplex with vertices corresponding to $\tilde{\rho}_{1}, \ldots, \tilde{\rho}_{m}$ approximates well the MM in the space of coefficients, so does the simplex of the transformed new vertices. In fact, it can be shown that the corrections of classicality in Eq. (21) are the same for both choices (see Sec. G2a of
Ref. [55]). We thus expect that the new vertices to be close to the those of metastable phases.

Indeed, it can be shown that the set of metastable phases is approximately invariant under the symmetries of the dynamics. In Sec. G2a of Ref. [55], we prove that the action of the symmetry on the metastable phases

$$
(\mathbf{U})_{k l} \equiv \operatorname{Tr}\left[\tilde{P}_{k} \mathcal{U}\left(\tilde{\rho}_{l}\right)\right]=\operatorname{Tr}\left[\tilde{P}_{k} \mathcal{U}_{\mathrm{MM}}\left(\tilde{\rho}_{l}\right)\right],
$$

$k, l=1, \ldots, m$, can be understood as an approximate permutation of metastable phases, that is,

$$
\left\|\mathbf{U}^{n}-\Pi^{n}\right\|_{1} \lesssim 3 \mathcal{C}_{\mathrm{cl}},
$$

where $\Pi$ is a permutation matrix and $n=1,2, \ldots$ are powers of the transformation [110]. Therefore, from Eq. (71) we obtain that $\tilde{\rho}_{l}$ is approximately transformed into $\pi^{n}(l)$ under symmetry applied $n$ times, $\left\|\mathcal{U}^{n}\left(\tilde{\rho}_{l}\right)-\tilde{\rho}_{\pi^{n}(l)}\right\| \lesssim 3 \mathcal{C}_{\mathrm{cl}}$, where $\pi$ is the permutation corresponding to $\Pi$. Similarly for $\rho_{l}$ being the closest state to $\tilde{\rho}_{l}$ we have $\left\|\mathcal{U}^{n}\left(\rho_{l}\right)-\rho_{\pi^{n}(l)}\right\| \lesssim$ $3 \mathcal{C}_{\mathrm{cl}}+2 \mathcal{C}_{+}$[cf. Eq. (12)]

\section{No continuous symmetries}

We now argue that any continuous weak symmetry acts trivially on the low-lying modes of the master operator when metastability is classical. A continuous weak symmetry is a symmetry $\left[\mathcal{U}_{\phi}, \mathcal{L}\right]=0$ [cf. Eq. (67)] for all $\phi$, where $\mathcal{U}_{\phi} \equiv$ $e^{\phi \mathcal{G}}$ with $\mathcal{G}(\rho) \equiv i[G, \rho]$ for a Hermitian operator $G$. For a small enough $\phi, \mathcal{U}_{\phi}$ is approximated by the identity transformation, and therefore for such values of $\phi$ we have $\boldsymbol{\Pi}=\mathbf{I}$ in Eq. (71) with $n=1$. Since, $\mathbf{U}_{\phi}^{n}=\mathbf{U}_{n \phi}$, from Eq. (71) the symmetry $\mathbf{U}_{\phi}$ is approximated by $\mathbf{I}$ for any $\phi$. But this is only possible when $\mathbf{U}_{\phi}=\mathbf{I}$, i.e., the symmetry leaves each metastable phase invariant, otherwise the corrections, as given by the Taylor series, could accumulate beyond $3 \mathcal{C}_{\mathrm{cl}} \ll 1$ (see Sec. G2b in Ref. [55] for a formal proof). Therefore, all slow eigenmodes of the dynamics must be invariant as well. As a corollary, we obtain that any nontrivial continuous symmetry of slow eigenmodes precludes classical metastability.

\section{Symmetric set of metastable phases}

We now show that the set of metastable phases can be chosen invariant under the action of a weak symmetry.

For a discrete symmetry, there exist a smallest nonzero integer $D$ such that $\mathcal{U}^{D} \mathcal{P}=\mathcal{P}$. We then have $\mathbf{U}^{D}=\mathbf{I}$, and thus from Eq. (71) also $\Pi^{D}=\mathbf{I}$. Let be $\pi$ be a permutation associated with $\boldsymbol{\Pi}$, that is, $(\boldsymbol{\Pi})_{k l}=\delta_{k \pi(l)}, k, l=1, \ldots, m$. For each cycle in the permutation, we choose an element $l$ and define

$$
\tilde{\rho}_{l}^{\prime} \equiv \frac{d_{l}}{D} \sum_{n=0}^{\frac{D}{d_{l}}-1} \mathcal{U}^{n d_{l}}\left(\tilde{\rho}_{l}\right),
$$

where $d_{l}$ is the length of the cycle $\pi^{d_{l}}(l)=l$ (and thus $D$ is divisible by $d_{l}$ ), while for the other elements of that cycle we define

$$
\tilde{\rho}_{\pi^{n}(l)}^{\prime} \equiv \mathcal{U}^{n}\left(\tilde{\rho}_{l}^{\prime}\right), \quad n=1, \ldots, d_{l}-1,
$$

and denote the transformation from the eigenmodes to this basis as $\mathbf{C}^{\prime}$ [cf. Eq. (18)]. This gives a symmetric set of metastable 
states,

$$
\mathcal{U}\left(\tilde{\rho}_{l}^{\prime}\right) \equiv \tilde{\rho}_{\pi(l)}^{\prime}, \quad l=1, \ldots, m,
$$

for which the distance to system states is again bounded by $\mathcal{C}_{+}$in Eq. (12). Furthermore, from Eq. (71) it can be shown that $\left\|\tilde{\rho}_{l}^{\prime}-\tilde{\rho}_{l}\right\| \lesssim 6 \mathcal{C}_{\mathrm{cl}}, l=1, \ldots, m$, and the corresponding corrections to the classicality, $\mathcal{C}_{\mathrm{cl}}^{\prime}$, defined analogously to Eq. (21), can increase at most by $\lesssim 6 \mathcal{C}_{\mathrm{cl}}$ (see Sec. G2c in Ref. [55] for the proofs). Therefore, without loss of generality, the set of metastable phases can be considered invariant under the symmetry. In Sec. VII A, we show how symmetric sets of $m$ candidate sets can be generated efficiently.

In the invariant basis of metastable phases, the action of the symmetry is exactly the permutation [see Fig. 5(b)]

$$
\mathbf{U}^{\prime} \equiv \Pi,
$$

where $\quad\left(\mathbf{U}^{\prime}\right)_{k l} \equiv \operatorname{Tr}\left[\tilde{P}_{k}^{\prime} \mathcal{U}\left(\tilde{\rho}_{l}^{\prime}\right)\right]=\operatorname{Tr}\left[\tilde{P}_{k}^{\prime} \mathcal{U}_{\mathrm{MM}}\left(\tilde{\rho}_{l}^{\prime}\right)\right], \quad k, l=$ $1, \ldots, m$, and $\tilde{P}_{l}^{\prime}$ is the dual basis to $\tilde{\rho}_{l}^{\prime}$ in Eqs. (72) and (73), $l=1, \ldots, m$; that is, $\operatorname{Tr}\left(\tilde{P}_{k}^{\prime} \tilde{\rho}_{l}^{\prime}\right)=\delta_{k l}$, from which it follows $\mathcal{U}\left(\tilde{P}_{l}^{\prime}\right)=\tilde{P}_{\pi(l)}^{\prime}$ [cf. Eq. (74)].

Finally, the set of corresponding basins of attraction is symmetric. That is, not only supports of the metastable phases in a cycle are connected by the symmetry operator $\mathcal{U}$, but also their basins of attractions. Indeed, for $\tilde{p}_{l}^{\prime}=\operatorname{Tr}\left[\tilde{P}_{l}^{\prime} \rho(0)\right]$, we have $\tilde{p}_{l}^{\prime}=\operatorname{Tr}\left\{\mathcal{U}\left(\tilde{P}_{l}^{\prime}\right) \mathcal{U}[\rho(0)]\right\}=\operatorname{Tr}\left\{\tilde{P}_{\pi(l)}^{\prime} \mathcal{U}[\rho(0)]\right\}, \quad l=$ $1, \ldots, m$. Thus, when $\rho(0)$ belongs to the basis of attraction of $\tilde{\rho}_{l}$, i.e., $\left|1-\tilde{p}_{l}^{\prime}\right| \ll 1, \mathcal{U}[\rho(0)]$ belongs to the basis of attraction of $\tilde{\rho}_{\pi(l)}$. Similarly, $\mathcal{U}\left(\mathbb{1}_{\mathcal{H}_{l}^{\prime}}\right)=\mathbb{1}_{\mathcal{H}_{\pi(l)}^{\prime}}$ for $\mathcal{H}_{l}^{\prime}$ defined for $\tilde{P}_{l}^{\prime}$ as in Eqs. (31)-(33).

\section{Symmetry of classical long-time dynamics}

Permutation symmetry. The weak symmetry of the longtime dynamics in Eq. (69) in the basis of the metastable phases reads

$$
[\tilde{\mathbf{W}}, \mathbf{U}]=0
$$

[cf. Eqs. (34) and (70)]. For the set of $m$ metastable phases chosen invariant under the symmetry [Eqs. (72) and (73)], the classical stochastic dynamics between metastable phases $\mathbf{W}^{\prime}$ that approximates the long-time-dynamics $\tilde{\mathbf{W}}^{\prime}[$ Eq. (36)] also features the weak symmetry with respect to the permutation П,

$$
\left[\tilde{\mathbf{W}}^{\prime}, \boldsymbol{\Pi}\right]=0=\left[\mathbf{W}^{\prime}, \boldsymbol{\Pi}\right]
$$

[cf. Eq. (75) and Fig. 5(b) and see Sec. G3a in Ref. [55] for the proof].

Structure of low-lying eigenmodes. We now show that, as a consequence of the symmetry in Eq. (77), the longtime dynamics may couple only plane waves over cycles of metastable phases with the same momentum, which results in the low-lying eigenmodes being their linear combinations. In particular, a unique stationary state is composed of uniform mixtures of states in cycles,

$$
\rho_{\mathrm{ss}}=\sum_{l}\left(\tilde{\mathbf{p}}_{\mathrm{ss}}^{\prime}\right)_{l} \frac{1}{d_{l}} \sum_{n=0}^{d_{l}-1} \tilde{\rho}_{\pi^{n}(l)}^{\prime},
$$

where $l$ runs over cycles representatives with $d_{l}$ denoting the length of the corresponding cycle [cf. Eqs. (72)-(74)], and $\left(\tilde{\mathbf{p}}_{\mathrm{ss}}^{\prime}\right)_{l}=\operatorname{Tr}\left(\tilde{P}_{l}^{\prime} \rho_{\mathrm{ss}}\right)$ corresponds to the stationary distribution of approximately classical dynamics of the symmetric degrees of freedom (see Fig. 5(a) and cf. Secs. E1c and G3b in Ref. [55]).

In the presence of a weak symmetry in Eq. (77), the longtime dynamics generator $\tilde{\mathbf{W}}^{\prime}$ is block diagonal in an eigenbasis of $\Pi$, which we can choose as plane waves over the cycles in the corresponding permutation $\pi$. Thus, the weak symmetry limits the number of free parameters of $\tilde{\mathbf{W}}^{\prime}$ to the sum of squared degeneracies of the symmetry eigenvalues, i.e., the plane-wave momenta (less 1 from the trace-preservation condition), and results in the eigenvectors of $\tilde{\mathbf{W}}^{\prime}$ being linear combination of the plane waves with the same momenta. In particular, $\tilde{\mathbf{W}}^{\prime}$ restricted to the symmetric plane waves, i.e., the uniform mixtures of metastable phases in each cycle, governs the long-time dynamics of symmetric states, which is tracepreserving and approximately positive with the corrections $\lesssim 2 \sqrt{\mathcal{C}_{\mathrm{cl}}^{\prime}}$ (see Sec. G3b in Ref. [55]).

Eigenvectors of $\tilde{\mathbf{W}}^{\prime}$ correspond directly to the low-lying eigenmodes of the master operator $\mathcal{L}$, as they determine the coefficients in the basis of the metastable phases [cf. Eqs. (72)-(74)],

$$
R_{k}=\sum_{l=1}^{m}\left(\mathbf{C}^{\prime-1}\right)_{k l} \tilde{\rho}_{l}^{\prime}, \quad L_{k}=\sum_{l=1}^{m}\left(\mathbf{C}^{\prime}\right)_{k l} \tilde{P}_{l}^{\prime},
$$

where $k=1, \ldots, m$. In particular, the left eigenvector of $\tilde{\mathbf{W}}^{\prime}$ corresponding to the eigenmode $L_{k}$ is simply the vector of $k$ th coefficient for the metastable phases [cf. Eq. (18)]. Analogously, the plane waves correspond to the eigenmodes of $\mathcal{U}_{\mathrm{MM}}$ [cf. Eq. (79)],

$$
\begin{aligned}
& R_{\pi^{j}(l)}^{\prime} \equiv \frac{1}{d_{l}} \sum_{n=0}^{d_{l}-1}\left(e^{-i 2 \pi \frac{j}{d_{l}}}\right)^{n} \tilde{\rho}_{\pi^{n}(l)}^{\prime}, \\
& L_{\pi^{j}(l)}^{\prime} \equiv \sum_{n=0}^{d_{l}-1}\left(e^{i 2 \pi \frac{j}{d_{l}}}\right)^{n} \tilde{P}_{\pi^{n}(l)}^{\prime},
\end{aligned}
$$

with $j=0,1, \ldots, d_{l}-1, d_{l}$ being the length of the considered cycle, and $e^{i 2 \pi j / d_{l}}$ the corresponding symmetry eigenvalue [cf. Eqs. (72) and (73)]. Therefore, the low-lying modes are their linear combinations,

$$
R_{k}=\sum_{l=1}^{m}\left(\mathbf{C}_{\mathbf{U}}^{-1}\right)_{k l} R_{l}^{\prime}, \quad L_{k}=\sum_{l=1}^{m}\left(\mathbf{C}_{\mathbf{U}}\right)_{k l} L_{l}^{\prime},
$$

where $\mathbf{C}_{\mathbf{U}}$ is the transformation from the basis of the low-lying eigenmodes to the basis of Eq. (80),

$$
\begin{array}{ll}
\left(\mathbf{C}_{\mathbf{U}}\right)_{k \pi^{j}(l)}=c_{k}^{\prime(l)} & \text { if } e^{i \phi_{k}}=e^{i 2 \pi \frac{j}{d_{l}}}, \\
\left(\mathbf{C}_{\mathbf{U}}\right)_{k \pi^{j}(l)}=0 & \text { otherwise, }
\end{array}
$$

with $k=1,2, \ldots, m, j=0, \ldots, d_{l}-1$, and $c_{k}^{\prime(l)}=\operatorname{Tr}\left(L_{k} \rho_{l}^{\prime}\right)$ [cf. Eq. (18) and Fig. 5(a)]. Importantly, $\mathbf{C}_{\mathbf{U}}$ is block diagonal in the eigenspaces of $\Pi$, so that $R_{k}$ and $L_{k}$ are only linear combinations of the eigenmatrices in Eq. (80) that fulfill $e^{i 2 \pi j / d_{l}}=e^{i \phi_{k}}$. In particular, the number of symmetric lowlying modes equals the number of cycles in the permutation, the corresponding block of $\mathbf{C}_{\mathbf{U}}$ is determined by coefficients for uniform mixtures of metastable phases in each cycle, and the symmetric stationary state is given by Eq. (78). Furthermore, when the symmetry eigenvalue $e^{i \phi_{k}}$ is unique among 
low-lying spectrum modes, $R_{k}$ and $L_{k}$ are necessarily proportional to Eqs. (80a) and (80b), but this is not the case for multiple cycles in general, e.g., for symmetric eigenmodes. For a single cycle, however, all symmetry eigenvalues are unique, so that $\mathbf{C}_{\mathbf{U}}$ is diagonal and determined by the coefficients of a single candidate phase. Thus, all low-lying eigenmodes are determined uniquely and the stationary state is an equal mixture of $m$ metastable phases [cf. Eq. (78)], as discussed in Ref. [42].

(No) conservation of symmetry. Finally, note that the symmetry of the MM can take place without the unitary operator $U$ being conserved during the initial relaxation and the metastable regime $\mathcal{U}, \mathcal{P}^{\dagger}(U)=U$, analogously as is the case for the symmetric manifold of stationary states $[61,63,111]$. Indeed, $U$ itself is a symmetric operator, $\mathcal{U}^{\dagger}(U)=U$, and thus, if conserved, it is spanned by the symmetric eigenmodes, $U=\sum_{l} u_{l} \sum_{n=0}^{d_{l}-1} \tilde{P}_{\pi^{n}(l)}$, where $u_{l} \equiv \operatorname{Tr}\left(U \tilde{\rho}_{l}\right)$ and $l$ runs over cycles representatives. For a MM with a single cycle, however, the conservation would lead to contradiction, as it would imply a trivial symmetry, $U \propto \mathbb{1}$, with $m$, rather than one, cycles.

\section{Symmetric test of classicality}

We now use the structure of eigenmodes of the dynamics in the presence of symmetry in Eqs. (81) and (82) to simplify the test of classicality introduced in Sec. III B. It is important to note beforehand that Eq. (80) forms a valid basis for any symmetric set of $m$ candidate states that are linearly independent. Thus, to verify whether candidate states indeed correspond to $m$ metastable phases, the test of classicality is necessary even in the case of a single cycle (see Sec. G4 in Ref. [55] for an example).

Exploiting the structure of the eigenmodes, the test of classicality can be simplified as follows. First, only coefficients of cycle representatives are needed to construct $\mathbf{C}_{\mathbf{U}}$ in Eq. (82). Second, as $\mathbf{C}_{\mathbf{U}}$ is block diagonal, to find the dual basis to the plane waves [Eq. (80b)], only matrices of the size of the permutation eigenspaces need to be inverted [112]. The dual basis to metastable phases in Eq. (20) can then be found by the inverse transformation to Eq. (80b), that is, with the coefficients as in Eq. (80a). Finally, to estimate the corrections to the classicality as in Eq. (23), it is enough to consider the elements of the dual basis corresponding to the chosen cycle representatives, $\tilde{P}_{l}^{\prime}$ [Eq. (80b) averaged over j], and multiply their contribution by $d_{l}$ [113].

\section{UNFOLDING CLASSICAL METASTABILITY NUMERICALLY}

With the theory of classical metastability now established, we turn to the question of how to efficiently uncover the structure of a MM and long-time dynamics in a given open quantum system governed by a master equation. We introduce two numerical methods to analyze the classical metastability in such systems with or without weak symmetries. The first approach in Sec. VII A requires diagonalizing the master operator and its low-lying eigenmodes. It verifies the presence of classical metastability, delivers the set of metastable phases, and uncovers the structure of the long-time dynamics. The second approach in Sec. VII B instead utilizes quantum trajec- tories with probabilities biased according to their activity, so that the metastable phases with the extreme activity are found.

\section{A. Metastable phases from master operator spectrum}

Efficient algorithms to uncover the structure of the stationary state manifold [114,115] (which utilize Ref. [116]; see also Ref. [117]) rely on the exact diagonalization of the master operator and the von Neumann algebra structure of the stationary left eigenmodes which arises when they are restricted to the maximal support of stationary states. This algebraic structure, however, is not generally present for the low-lying left eigenmodes, as visible, e.g., in first-order corrections to the formerly stationary modes when they are perturbed away from a dissipative phase transition at a finite system size by excitation of decaying modes (see Supplemental Material of Refs. [40]).

Here, we introduce a general approach which delivers the set metastable phases and the structure of the long-time dynamics when the metastability is classical. Similarly to the algorithm in Refs. [114,115], it is based on the low-lying left eigenmodes of the master operator in Eq. (1), but their connection to basins of attractions is utilized by the observation that extreme values of the corresponding coefficients are achieved by metastable phases (cf. Fig. 2), which thus correspond to the projections on the MM of the extreme eigenstates of left eigenmodes. To guarantee that all metastable phases are found, random rotations of low-lying modes are employed until the corrections to the classicality are small. This way, the algorithm remains efficient, as it does not simply probe the whole space system space of pure states (cf., e.g., Ref. [118]). Furthermore, symmetries of the dynamics can be exploited to simplify the method. We also consider how observable averages distinguishing metastable phases (i.e., order parameters) can be utilized. Finally, we note that this method has been recently successfully applied in Ref. [54] to the open quantum East model [50] featuring a hierarchy of metastabilities and translation symmetry.

\section{Metastable phases construction}

Our approach consists of the following steps (see also Fig. 6):

(1) Diagonalize $\mathcal{L}$ to find the left eigenmatrices $L_{k}$ below the gap in the spectrum, $k=2, \ldots, m$.

(2) Construct candidate metastable states:

(i) diagonalize the (rotated) eigenmatrices $L_{k}$,

(ii) choose the eigenstates associated to their extreme eigenvalues as initial states for candidate metastable states,

(iii) discard repetitions in candidate metastable statescluster in the coefficients space.

(3) Find best candidate metastable states:

(i) If the number of candidate states $\geqslant m$, then choose the set of $m$ states providing the simplex with the largest volume, i.e., the largest $|\operatorname{det} \mathbf{C}|[\mathrm{cf} . \mathrm{Eq} .(18)]$ and calculate the corresponding corrections to the classicality in Eq. (21), as can be easily bounded by Eq. (23).

(ii) If the number of candidate states $<m$, or the corrections to the classicality in Step 3i are not negligible, then enlarge the set of candidates obtained from Step 3 by 


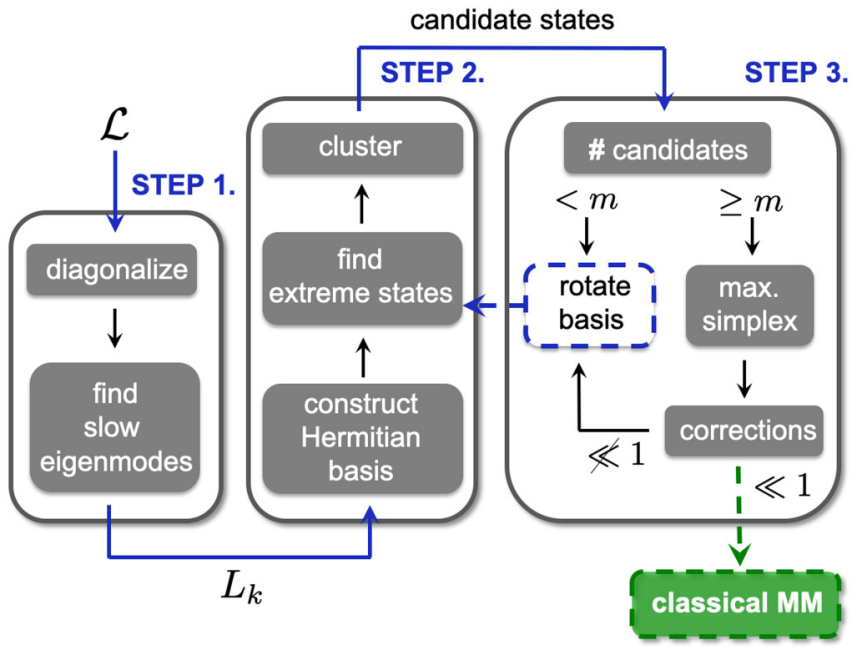

FIG. 6. Metastable phases construction. We sketch the approach to construct a candidate set of $m$ metastable phases within a given MM that provides the best classical approximation in terms of corrections in Eq. (21). The construction can be refined by considering symmetries of the dynamics.

considering a random rotation of the basis of the left eigenmatrices in Step 2.

Step 1 in the above construction provides the lowdimensional description of the MM, and, as explained in Sec. III, allows for testing the approximation of the MM as mixtures of $m$ candidate states. We choose Hermitian $L_{k}$ replacing conjugate pairs of eigenmodes $L_{k}, L_{k}^{\dagger}$ [69] by

$$
L_{k}^{R} \equiv \frac{e^{-i \varphi_{k}} L_{k}+e^{i \varphi_{k}} L_{k}^{\dagger}}{\sqrt{2}}, \quad L_{k}^{I} \equiv \frac{e^{-i \varphi_{k}} L_{k}-e^{i \varphi_{k}} L_{k}^{\dagger}}{\sqrt{2} i},
$$

where $e^{-i \varphi_{k}}$ is an arbitrary phase. Step 2 relies on the result in Sec. H1 of Ref. [55], that metastable states arising from extreme eigenstates of the dynamics eigenmodes can be used to approximate metastable phases in classical MMs, as long as only a single metastable phase is close to the extreme value of the corresponding coefficient $c_{k}$ (which we refer to as the case without degeneracy); cf. Figs. 2 and 5(a). We then discard any repetitions in the set of candidate states (to treat all coefficients on equal footing, we set the normalization $c_{k}^{\max }-c_{k}^{\min }=1$, where $c_{k}^{\max }$ and $c_{k}^{\min }$ are extreme eigenvalues of $\left.L_{k}\right)$. Indeed, for a given left basis we obtain $2(m-1)$ candidate metastable states corresponding to $2(m-1)$ extreme eigenvalues of the basis elements, which may provide up to $m$ metastable phases. In the case without degeneracy, each candidate corresponds to one of $m$ metastable phases. In the case with degeneracy, some of extreme eigenstates may correspond to mixtures of metastable phases: provided that the set of candidate states features all metastable phases, such a candidate state should be discarded in Step 3i (this relies on the result from Sec. H3 in Ref. [55], that the simplex of metastable phase is approximately the largest simplex inside the MM; cf. Fig. 2). However, such mixtures may cause less than $m$ candidates to remain after clustering, or result in a set of phases which provides a poor approximation to the true MM; even without degeneracy, it is possible that some metastable phases may reside on the interior of the hyper- cube defined by the extreme values of the coefficients, and as such will not appear in the set of candidate states taken from extreme eigenvalues of the eigenmodes. Nevertheless, random rotations in Step 3ii ensure that each metastable phase is eventually exposed, i.e., a basis in which that metastable phase achieves an extreme coefficient value without degeneracy is eventually considered (with the probability 1 achieved exponentially in the number of rotations; cf. Sec. H2 in Ref. [55]). When the set of metastable phases leading to small corrections to the classicality is found, the dual basis in Eq. (20) can be constructed, and the long-time dynamics decomposed as in Eq. (34).

Naturally, instead of considering distances between candidate states in the space of coefficients, as used in Steps 2 and $3 \mathrm{i}$, candidate states can be clustered with respect to the trace distance in the space of density matrices, while the best $m$ candidate states can be chosen to achieve minimal corrections to the classicality instead of the maximal simplex volume. These modifications, however, require working with operators on the system Hilbert space, rather than on the space of coefficients, and thus are in general more expensive numerically [for classical MMs, $m \leqslant \operatorname{dim}(\mathcal{H})$ ], while not necessary for MMs with nonnegligible volumes (cf. Secs. H2 and H3 in Ref. [55]).

Our approach will not deliver a set of metastable phases with negligible corrections to the classicality whenever the MM is not classical. In particular, quantum MMs [40] featuring decoherence free subspaces [74-76] or noiseless subsystems [77,78]: which, e.g., at $m=4$ amount to Blochsphere in the coefficient space, rather than a tetrahedron (see also Ref. [119]), cannot be approximated as probabilistic mixtures of $m$ metastable phases. Even for classical metastability emerging in many-body open quantum systems, the approach relies on the condition in Eq. (25), which may be fulfilled only at larger system sizes, when the low-lying part of the master operator spectrum to be sufficiently separated from the fast modes. In this case, our approach may not succeed for smaller system sizes with less pronounced metastability.

While degeneracies are unlikely to occur in a generic model, they typically appear in the presence a hierarchy of metastabilities or as a consequence of symmetries of the dynamics, which we discuss in detail below. In particular, in the presence of a weak symmetry, not only the degeneracy can be efficiently remedied, but also the search for candidates states made even more efficient.

\section{Construction for hierarchy of metastable manifolds}

In the presence of hierarchy of metastabilities with a further separation at $m_{2}<m$ in the spectrum of the master operator, the degeneracy appears as a consequence of the fact that the simplex of $m$ metastable phases of the first $\mathrm{MM}$, when projected onto the coefficients $\left(c_{2}, \ldots, c_{m_{2}}\right)$, is approximated by a simplex with $m_{2}$ vertices corresponding to $m_{2}$ metastable phases of the second MM. This requires (at least) $m_{2}$ metastable phases in the first MM to evolve directly into $m_{2}$ metastable phases of the second MM. Each of other $m-m_{2}$ metastable phases of the first MM either evolves into a single phase of the second MM, or it belongs to the decay subspace in which case it in general evolves into a mixture of 
$m_{2}$ metastable phases of the second MM. In the former case, the metastable phases in the first MM that evolve into the same phase in the second MM are degenerate in the coefficients $\left(c_{2}, \ldots, c_{m_{2}}\right)$. In the latter case, they do cannot take extreme values of those coefficients, even after a rotation of the first $m_{2}$ modes (see Secs. F1 and H4 in Ref. [55]). Nevertheless, rotations of all $m$ modes allow both for the degeneracy to be lifted and for every metastable phase to take an extreme value in one of the coefficients.

\section{Construction for metastable manifolds with symmetries}

In the presence of a weak symmetry $\mathcal{U}$ [Eq. (67)], the master operator $\mathcal{L}$ is block diagonal in the eigenspaces of $\mathcal{U}$, which simplifies its diagonalization [61-63]. As a consequence, the low-lying eigenmodes of the dynamics are chosen as linear combinations of the plane waves over the cycles induced by the symmetry on $m$ metastable phases [cf. Eq. (81)]. In particular, for an eigenmode $L_{k}, k=2, \ldots, m$ with a symmetry eigenvalue $e^{i \phi_{k}}, \mathcal{U}^{\dagger}\left(L_{k}\right)=e^{i \phi_{k}} L_{k}$, and the minimal integer $n_{k}>0$ leading to $e^{i n_{k} \phi_{k}}=1, L_{k}$ is supported on cycles with the length equal $n_{k}$ or larger but divisible by $n_{k}$. The latter case, of subcycles with length $n_{k}$, leads to degeneracy of the coefficient $c_{k}$ for the metastable phases connected by $\mathcal{U}^{n_{k}}$, with its extreme eigenstates generically projecting onto their uniform mixture [cf. Eq. (80b) and Fig. 5(a), where $\tilde{\rho}_{1}$ and $\tilde{\rho}_{3}$ are degenerate in $c_{2}$ and $\left.c_{4}\left(n_{2}=n_{4}=1\right)\right]$. Nevertheless, coefficient degeneracy can be remedied and the structure of the low-lying eigenmodes can be used to actually enhance the introduced approach, as we now explain (see also Sec. H5 in Ref. [55]).

We note that when $\operatorname{GCF}\left(n_{k}, n_{l}\right)<n_{k}$ for all $n_{l} \neq n_{k}, k, l=$ $2, \ldots, m$, the eigenmode $L_{k}$ is supported only on cycles of length $n_{k}$, as there are no longer cycles with the length divisible by $n_{k}$. The symmetry $\mathcal{U}^{n_{k}}$ then acts trivially on the corresponding metastable phases and the above discussed degeneracy is absent. Analogously to the general case, any plane wave in $L_{k}$ can then be exposed by random rotations of the eigenmodes with the same symmetry eigenvalue $e^{i \phi_{k}}$, while at least a single metastable phase from each cycle can be obtained by considering extreme eigenstates of both $L_{k}^{R}$ and $L_{k}^{I}$ [120]. Other metastable phases in the considered cycles can be recovered by applying the symmetry $n_{k}-1$ times, so there is no need to consider eigenmodes $L_{l}$ with a different symmetry eigenvalue supported on the same cycles (i.e., $L_{l}$ with $e^{i \phi_{k}} \neq e^{i \phi_{k}}$ but $n_{l}=n_{k}$ ).

Furthermore, metastable phases in cycles with the length corresponding to subcycles, e.g., invariant metastable phases, can also be found. For $L_{l}$ such that $n_{l}$ is divides only $n_{k}>n_{l}$ for the above considered eigenmodes $L_{k}$, when the degeneracy of $e^{i \phi_{l}}$ equals the number of already considered cycles with $n_{k}$ divisible by $n_{l}$ (i.e., the sum of the corresponding symmetry eigenvalue degeneracy for all such $n_{k}$ values), $L_{l}$ is supported on the already considered cycles. Otherwise, $L_{l}$ features new cycles with the length $n_{l}$, which can be unfolded, as before, by rotations of all eigenmodes with the same symmetry eigenvalue as $e^{i \phi_{l}}$ and considering both $L_{l}^{R}$ and $L_{l}^{I}$ [120]. Here, equal mixtures of already considered phases connected by $\mathcal{U}^{n_{k} / n_{l}}$ will also be found, but such candidate states will not lead to the maximal volume simplex. Again, by applying symmetry $\mathcal{U}$ the full (sub)cycles can be recovered, and other eigenmodes $L_{j}$ with $n_{j}=n_{l}$, but a different symmetry eigenvalue can be discarded. Analogous results hold for the remaining eigenmodes, but with respect to $L_{l}$ and $e^{i \phi_{l}}$ degeneracy.

In summary, the set of eigenmodes considered in Step 2 is significantly reduced, with its size equal to the number of cycles and the subcycles with other cycle's length. Furthermore, only rotations of eigenmodes with the same symmetry eigenvalue are necessary in Step 3ii. Importantly, as the eigenstates of $L_{k}^{R}$ and $L_{k}^{I}$ and the corresponding metastable states are invariant under $\mathcal{U}^{n_{k}}$, that is, generate cycles of candidate states with the length dividing $n_{k}$, following the prescription above, we arrive at an invariant set of candidate states. This invariance can be maintained by clustering whole cycles of candidate states rather than individual states in Step 2. Then, without loss of generality, in Step 3i, instead of considering subsets of all candidate states, we can choose candidate states as sets of cycles with their lengths summing to $m$. In that case, the volume of the simplex can be efficiently calculated as $\left|\operatorname{det} \mathbf{C}_{\mathbf{U}}\right| /(m-1) ! \prod_{l} \sqrt{d_{l}}$, where $\mathbf{C}_{\mathbf{U}}$ is the block-diagonal matrix in Eq. (82) and the product, which runs over cycle representatives, is the same for all sets of linearly independent candidates [112], while the corrections to the classicality can be efficiently calculated with the symmetric test of classicality of Sec. VIB 5.

\section{Construction utilizing order parameters}

Instead of considering the eigenmodes of the dynamics, we can choose a left eigenbasis formed by a set of $m$ observables $O_{l}, l=1, \ldots, m$ projected onto the lowlying eigenmodes, i.e., $\mathcal{P}^{\dagger}\left(O_{l}\right)=\sum_{k=1}^{m} b_{k}^{(l)} L_{k}$, where $b_{k}^{(l)} \equiv$ $\operatorname{Tr}\left(O_{l} R_{k}\right)$ [cf. Eq. (4)], provided that those projections are linearly independent. In this case, the extreme eigenstates of $\mathcal{P}^{\dagger}\left(O_{k}\right)$ will give metastable states attaining extreme values in the average of the observable $O_{k}$. Those metastable states will correspond to metastable phases, up to degeneracy of metastable phase averages of $O_{k}$ (in particular, in the presence of nontrivial weak symmetry of low-lying eigenmodes, it is necessary to consider observables breaking the symmetry). Among others, this can be helpful when the volume of MM in the space of coefficients is negligible. In the next section, we extend this approach by considering continuous measurements instead of system observables.

\section{B. Metastable phases from biased quantum trajectories}

In some systems, the metastability can be a collective effect emerging as the system size increases [41]. If large system sizes are required for prominent metastability, then exact diagonalization may not be feasible. Therefore, we now introduce an alternative numerical approach to finding metastable phases in classical MMs using QJMC simulations [8-12] and biased sampling in the framework of large deviation theory (see Ref. [121] for a review). In classical stochastic dynamics biased sampling can be efficiently incorporated into the generation of trajectories, with techniques such as transition path sampling [122] and cloning [123].

Trajectories of the biased master equation $\mathcal{L}_{s}$ in Eq. (44) can be viewed as trajectories of $\mathcal{L}$ with their probability multiplied by $e^{-s K(t)}$, where $K(t)$ is the total number of jumps 
occurring in a quantum trajectory of length $t$. The maximal eigenmode $\rho_{\mathrm{ss}}(s)$ of $\mathcal{L}_{s}$ corresponds then to the asymptotic system state in quantum trajectories averaged with the biased probability. In Sec. V B 5, we argued that $\rho_{\mathrm{ss}}(s)$ can approximate metastable phases of extreme activity for appropriately chosen $s$ when the activity dominates the transition rates of long-time dynamics [cf. Figs. 4(a) and 4(c)]. Thus, if the efficient biased sampling could be generalized to QJMC sampling, metastable phases with extreme activity could be accessed via time-average of a biased trajectory over timelength within the metastable regime [124].

Similarly as in the case of degeneracy of coefficients, when more than a single metastable phase corresponds to the maximum or the minimum activity, $\rho_{\mathrm{ss}}(s)$ corresponds to a mixture of the metastable phases with the extreme value (e.g., when both $\mathcal{L}$ and $\mathcal{J}$ obey a symmetry that is broken in the MM, the mixture is symmetric). Nevertheless, the discussion in Sec. V B 5 is analogous for the activity of individual jumps, and thus a further distinction between metastable phases can be enabled this way, e.g., by breaking the translation symmetry of $\mathcal{L}_{s}$ in the case of identical local jumps (see Sec. E3a in Ref. [55]). Finally, we note that in the case of general metastability, this approach will return the metastable states corresponding to the extreme values of jump activity.

\section{CONCLUSIONS AND OUTLOOK}

In this paper, we formulated a comprehensive theory for the emergence of classical metastability for open quantum systems whose dynamics is governed by a master operator. We showed that classical metastability is characterized by the approximation of metastable states as probabilistic mixtures of $m$ metastable phases, where $m$ is the number of low-lying modes of the master operator. Namely, in terms of the corresponding corrections, metastable phases are approximately disjoint, while the long-time dynamics, both on average and in individual quantum trajectories, is approximately governed by an effective classical stochastic generator. Furthermore, any nontrivial weak symmetries present at long times are necessarily discrete as they correspond to approximate permutations of metastable phases, under which the classical dynamics is invariant. To investigate metastability for a given open quantum system, we introduced the test of classicality - an approach to verify the approximation of the MM by a set of candidate metastable phases. We also developed a complementary numerical approach to deliver sets of candidate metastable phases. Since that approach requires diagonalization of the master operator - a difficult task in systems of larger size-we also discussed an alternative based on the concept of biased trajectory sampling.

The techniques we introduced here allow us to achieve a complete understanding of classical metastability emerging in an open quantum system. A concrete application of the methods described here to a many-body system of an open quantum East model [50] is given in Ref. [54], where despite the stationary state being analytic in dynamics parameters, the dynamics is found to feature a hierarchy of classical metastabilities, with metastable phases breaking the translation symmetry of the model and their number increasing with the system size. This structure is then shown to be analogous to metastability in the classical East model, but with an effective temperature and coherent excitations.

While our general approach relies only on the Markovian approximation of system dynamics, non-Markovian effects could be included via the chain partial diagonalization [125-127] (see also Ref. [128]). However, an open quantum system and its environment form an isolated system, which raises a question about the relation of classical metastability to separation of timescales in closed system dynamics, which generically leads to prethermalization of subsystem states [129]. Furthermore, as in this work no assumptions were made on the structure of system Hamiltonian or jump operators, it should be explored how metastable phases and their basins of attraction can be further characterized in systems with local interactions and local dissipation (see also Ref. [25]). Finally, it remains an open question what is the structure of general quantum metastability and how it can be efficiently investigated, e.g., to uncover metastable coherences [40]. Extending the methods described here to this general case would inform, among others, the study of a general structure of first-order dissipative phase transitions in open quantum systems.

\section{ACKNOWLEDGMENTS}

K.M. thanks M. Guţă for helpful discussions. K.M. gratefully acknowledges support from a Henslow Research Fellowship. We acknowledge financial support from EPSRC Grant No. EP/R04421X/1, from the H2020-FETPROACT2014 Grant No. 640378 (RYSQ), and by University of Nottingham Grant No. FiF1/3. I.L. acknowledges support by the "Wissenschaftler-Rückkehrprogramm GSO/CZS" of the Carl-Zeiss-Stiftung, through the Deutsche Forschungsgemeinsschaft (DFG, German Research Foundation) under Project No. 435696605 and through the European Union's H2020 research and innovation programme [Grant Agreement No. 800942 (ErBeStA)]. We are grateful for access to the University of Nottingham Augusta HPC service.
[1] J. D. Pritchard, D. Maxwell, A. Gauguet, K. J. Weatherill, M. P. A. Jones, and C. S. Adams, Cooperative Atom-Light Interaction in a Blockaded Rydberg Ensemble, Phys. Rev. Lett. 105, 193603 (2010).

[2] R. Blatt and C. F. Roos, Quantum simulations with trapped ions, Nat. Phys. 8, 277 (2012).

[3] J. W. Britton, B. C. Sawyer, A. C. Keith, C. C. J. Wang, J. K. Freericks, H. Uys, M. J. Biercuk, and J. J. Bollinger, Engineered two-dimensional Ising interactions in a trapped-ion quantum simulator with hundreds of spins, Nature (London) 484, 489 (2012).

[4] Y. O. Dudin and A. Kuzmich, Strongly interacting Rydberg excitations of a cold atomic gas, Science 336, 887 (2012).

[5] T. Peyronel, O. Firstenberg, Q. Liang, S. Hofferberth, A. V. Gorshkov, T. Pohl, M. D. Lukin, and V. Vuletic, Quantum nonlinear optics with single photons enabled by strongly interacting atoms, Nature (London) 488, 57 (2012). 
[6] G. Günter, H. Schempp, M. Robert-de Saint-Vincent, V. Gavryusev, S. Helmrich, C. S. Hofmann, S. Whitlock, and M. Weidemüller, Observing the dynamics of dipole-mediated energy transport by interaction-enhanced imaging, Science 342, 954 (2013).

[7] S. Schmidt and J. Koch, Circuit QED lattices: Towards quantum simulation with superconducting circuits, Ann. Phys. 525, 395 (2013)

[8] J. Dalibard, Y. Castin, and K. Mølmer, Wave-Function Approach to Dissipative Processes in Quantum Optics, Phys. Rev. Lett. 68, 580 (1992).

[9] R. Dum, A. S. Parkins, P. Zoller, and C. W. Gardiner, Monte Carlo simulation of master equations in quantum optics for vacuum, thermal, and squeezed reservoirs, Phys. Rev. A 46, 4382 (1992).

[10] K. Mølmer, Y. Castin, and J. Dalibard, Monte Carlo wavefunction method in quantum optics, J. Opt. Soc. Am. B 10, 524 (1993).

[11] M. B. Plenio and P. L. Knight, The quantum-jump approach to dissipative dynamics in quantum optics, Rev. Mod. Phys. 70, 101 (1998).

[12] A. J. Daley, Quantum trajectories and open many-body quantum systems, Adv. Phys. 63, 77 (2014).

[13] A. A. Gangat, T. I, and Y.-J. Kao, Steady States of Infinite-Size Dissipative Quantum Chains Via Imaginary Time Evolution, Phys. Rev. Lett. 119, 010501 (2017).

[14] E. G. D. Torre, S. Diehl, M. D. Lukin, S. Sachdev, and P. Strack, Keldysh approach for nonequilibrium phase transitions in quantum optics: Beyond the Dicke model in optical cavities, Phys. Rev. A 87, 023831 (2013).

[15] L. M. Sieberer, M. Buchhold, and S. Diehl, Keldysh field theory for driven open quantum systems, Rep. Prog. Phys. 79, 096001 (2016).

[16] M. F. Maghrebi and A. V. Gorshkov, Nonequilibrium manybody steady states via Keldysh formalism, Phys. Rev. B 93, 014307 (2016).

[17] J. J. Mendoza-Arenas, S. R. Clark, S. Felicetti, G. Romero, E. Solano, D. G. Angelakis, and D. Jaksch, Beyond mean-field bistability in driven-dissipative lattices: Bunchingantibunching transition and quantum simulation, Phys. Rev. A 93, 023821 (2016).

[18] M. Foss-Feig, P. Niroula, J. T. Young, M. Hafezi, A. V. Gorshkov, R. M. Wilson, and M. F. Maghrebi, Emergent equilibrium in many-body optical bistability, Phys. Rev. A 95, 043826 (2017)

[19] W. Casteels and M. Wouters, Optically bistable drivendissipative Bose-Hubbard dimer: Gutzwiller approaches and entanglement, Phys. Rev. A 95, 043833 (2017).

[20] F. Letscher, O. Thomas, T. Niederprüm, M. Fleischhauer, and H. Ott, Bistability Versus Metastability in Driven Dissipative Rydberg Gases, Phys. Rev. X 7, 021020 (2017).

[21] J. Jin, A. Biella, O. Viyuela, C. Ciuti, R. Fazio, and D. Rossini, Phase diagram of the dissipative quantum Ising model on a square lattice, Phys. Rev. B 98, 241108(R) (2018).

[22] N. R. de Melo, C. G. Wade, N. Šibalić, J. M. Kondo, C. S. Adams, and K. J. Weatherill, Intrinsic optical bistability in a strongly driven Rydberg ensemble, Phys. Rev. A 93, 063863 (2016).

[23] S. R. K. Rodriguez, W. Casteels, F. Storme, N. Carlon Zambon, I. Sagnes, L. Le Gratiet, E. Galopin, A. Lemaître,
A. Amo, C. Ciuti, and J. Bloch, Probing a Dissipative Phase Transition Via Dynamical Optical Hysteresis, Phys. Rev. Lett. 118, 247402 (2017).

[24] C. Ates, B. Olmos, J. P. Garrahan, and I. Lesanovsky, Dynamical phases and intermittency of the dissipative quantum Ising model, Phys. Rev. A 85, 043620 (2012).

[25] H. Landa, M. Schiró, and G. Misguich, Multistability of Driven-Dissipative Quantum Spins, Phys. Rev. Lett. 124, 043601 (2020).

[26] H. Weimer, Variational Principle for Steady States of Dissipative Quantum Many-Body Systems, Phys. Rev. Lett. 114, 040402 (2015).

[27] H. Weimer, Variational analysis of driven-dissipative Rydberg gases, Phys. Rev. A 91, 063401 (2015).

[28] V. R. Overbeck, M. F. Maghrebi, A. V. Gorshkov, and H. Weimer, Multicritical behavior in dissipative Ising models, Phys. Rev. A 95, 042133 (2017).

[29] M. Biondi, S. Lienhard, G. Blatter, H. E. Türeci, and S. Schmidt, Spatial correlations in driven-dissipative photonic lattices, New J. Phys. 19, 125016 (2017).

[30] H. Spohn, An algebraic condition for the approach to equilibrium of an open N-level system, Lett. Math. Phys. 2, 33 (1977).

[31] D. E. Evans, Irreducible quantum dynamical semigroups, Commun. Math. Phys. 54, 293 (1977).

[32] S. G. Schirmer and X. Wang, Stabilizing open quantum systems by Markovian reservoir engineering, Phys. Rev. A 81, 062306 (2010).

[33] D. Nigro, On the uniqueness of the steady-state solution of the Lindblad-Gorini-Kossakowski-Sudarshan equation, J. Stat. Mech. (2019) 043202.

[34] P. M. Chaikin and T. C. Lubensky, Principles of Condensed Matter Physics (Cambridge University Press, Cambridge, UK, 2000), Vol. 1.

[35] B. Gaveau and L. S. Schulman, Dynamical metastability, J. Phys. A 20, 2865 (1987).

[36] B. Gaveau and L. S. Schulman, Theory of nonequilibrium first-order phase transitions for stochastic dynamics, J. Mat. Phys. 39, 1517 (1998).

[37] A. Bovier, M. Eckhoff, V. Gayrard, and M. Klein, Metastability and low lying spectra in reversible Markov chains, Commun. Math. Phys. 228, 219 (2002).

[38] B. Gaveau and L. S. Schulman, Multiple phases in stochastic dynamics: Geometry and probabilities, Phys. Rev. E 73, 036124 (2006).

[39] J. Kurchan, Six out of equilibrium lectures, arXiv:0901.1271.

[40] K. Macieszczak, M. Guta, I. Lesanovsky, and J. P. Garrahan, Towards a Theory of Metastability in Open Quantum Dynamics, Phys. Rev. Lett. 116, 240404 (2016).

[41] D. C. Rose, K. Macieszczak, I. Lesanovsky, and J. P. Garrahan, Metastability in an open quantum Ising model, Phys. Rev. E 94, 052132 (2016).

[42] F. Minganti, A. Biella, N. Bartolo, and C. Ciuti, Spectral theory of Liouvillians for dissipative phase transitions, Phys. Rev. A 98, 042118 (2018).

[43] J. Jäckle and S. Eisinger, A hierarchically constrained kinetic Ising model, Z. Phys. B 84, 115 (1991).

[44] P. Sollich and M. R. Evans, Glassy Timescale Divergence and Anomalous Coarsening in a Kinetically Constrained Spin Chain, Phys. Rev. Lett. 83, 3238 (1999). 
[45] J. P. Garrahan and D. Chandler, Geometrical Explanation and Scaling of Dynamical Heterogeneities in Glass Forming Systems, Phys. Rev. Lett. 89, 035704 (2002).

[46] P. Sollich and M. R. Evans, Glassy dynamics in the asymmetrically constrained kinetic Ising chain, Phys. Rev. E 68, 031504 (2003).

[47] K. Binder and W. Kob, Glassy Materials and Disordered Solids: An Introduction to Their Statistical Mechanics (Revised Edition) (World Scientific, Singapore, 2011).

[48] G. Biroli and J. P. Garrahan, Perspective: The glass transition, J. Chem. Phys. 138, 12A301 (2013).

[49] K. Binder and A. P. Young, Spin glasses: Experimental facts, theoretical concepts, and open questions, Rev. Mod. Phys. 58, 801 (1986).

[50] B. Olmos, I. Lesanovsky, and J. P. Garrahan, Facilitated Spin Models of Dissipative Quantum Glasses, Phys. Rev. Lett. 109, 020403 (2012).

[51] I. Lesanovsky, M. van Horssen, M. Guţă, and J. P. Garrahan, Characterization of Dynamical Phase Transitions in Quantum Jump Trajectories Beyond the Properties of the Stationary State, Phys. Rev. Lett. 110, 150401 (2013).

[52] B. Olmos, I. Lesanovsky, and J. P. Garrahan, Outof-equilibrium evolution of kinetically constrained manybody quantum systems under purely dissipative dynamics, Phys. Rev. E 90, 042147 (2014).

[53] L. F. Cugliandolo and G. Lozano, Real-time nonequilibrium dynamics of quantum glassy systems, Phys. Rev. B 59, 915 (1999).

[54] D. C. Rose, K. Macieszczak, I. Lesanovsky, and J. P. Garrahan, Hierarchical classical metastability in an open quantum East model, arXiv:2010.15304.

[55] See Supplemental Material at http://link.aps.org/supplemental/ 10.1103/PhysRevResearch.3.033047 for detailed proofs of the results throughout the paper.

[56] J. Thingna, D. Manzano, and J. Cao, Dynamical signatures of molecular symmetries in nonequilibrium quantum transport, Sci. Rep. 6, 28027 (2016).

[57] G. Lindblad, On the generators of quantum dynamical semigroups, Commun. Math. Phys. 48, 119 (1976).

[58] V. Gorini, A. Kossakowski, and E. C. G. Sudarshan, Completely positive dynamical semigroups of N-level systems, J. Math. Phys. 17, 821 (1976).

[59] C. W. Gardiner and P. Zoller, Quantum Noise, 3rd ed., Complexity (Springer, Berlin, 2004).

[60] J. P. Garrahan and I. Lesanovsky, Thermodynamics of Quantum Jump Trajectories, Phys. Rev. Lett. 104, 160601 (2010).

[61] B. Baumgartner and H. Narnhofer, Analysis of quantum semigroups with GKS-Lindblad generators: II. General, J. Phys. A 41, 395303 (2008).

[62] B. Buča and T. Prosen, A note on symmetry reductions of the Lindblad equation: Transport in constrained open spin chains, New J. Phys. 14, 073007 (2012).

[63] V. V. Albert and L. Jiang, Symmetries and conserved quantities in Lindblad master equations, Phys. Rev. A 89, 022118 (2014).

[64] J. P. Garrahan, A. D. Armour, and I. Lesanovsky, Quantum trajectory phase transitions in the micromaser, Phys. Rev. E 84, 021115 (2011)

[65] F. Carollo, J. P. Garrahan, I. Lesanovsky, and C. PérezEspigares, Making rare events typical in Markovian open quantum systems, Phys. Rev. A 98, 010103(R) (2018).
[66] It is possible that the master operator may not be completely diagonalizable, in which this case its simplest form is the Jordan normal form. Under exponentiation, this gives rise to a polynomial dependence on the time, however this is accompanied by the usual exponential evolution. Our results in Secs. III, IV, V A, VI, and VII carry directly to that case by assuming that after the initial relaxation, any polynomial evolution of fast modes is dominated by the decaying exponential and can be neglected [cf. Eq. (3)], while during the metastable regime dynamics of the $m$ slow modes can be replaced by no evolution [cf. Eq. (4)]. In Sec. V B, the corrections to Eqs. (54) and (61) need to be adjusted, together with corresponding Secs. E3e, E3f, and E3h in Ref. [55].

[67] V. V. Albert, B. Bradlyn, M. Fraas, and L. Jiang, Geometry and Response of Lindbladians, Phys. Rev. X 6, 041031 (2016).

[68] B. Bellomo, G. L. Giorgi, G. M. Palma, and R. Zambrini, Quantum synchronization as a local signature of super- and subradiance, Phys. Rev. A 95, 043807 (2017).

[69] Since $\mathcal{L}$ is Hermiticity preserving, if $R_{k}\left(L_{k}\right)$ is a right (left) eigenmode of the dynamics with an eigenvalue $\lambda_{k}$, so is $R_{k}^{\dagger}$ $\left(L_{k}^{\dagger}\right)$ with the eigenvalue $\lambda_{k}^{*}$. Thus the set of low-lying modes is invariant under the Hermitian conjugation.

[70] B. Sciolla, D. Poletti, and C. Kollath, Two-Time Correlations Probing the Dynamics of Dissipative Many-Body Quantum Systems: Aging and Fast Relaxation, Phys. Rev. Lett. 114, 170401 (2015).

[71] We restrict the discussion to the real space of Hermitian operators. In this case, the induced norm of a superoperator can be shown to be achieved for a pure state (i.e., a rank one operator). Let $X$ be a Hermitian operator with eigenvalues $x_{n}$ and projections on the corresponding eigenstates denoted as $\rho_{n}$. For a superoperator $\mathcal{Y}$, we have $\|\mathcal{Y}[X]\| /\|X\| \leqslant$ $\left(\sum_{n}\left|x_{n}\right||| \mathcal{Y}\left[\rho_{n}\right]||\right) /\left(\sum_{n}\left|x_{n}\right|\right) \leqslant \max _{n}|| \mathcal{Y}\left[\rho_{n}\right]||$.

[72] For an integer $n \geqslant 1$ such that $t^{\prime \prime} \leqslant t_{n} \equiv t / n \leqslant t^{\prime}$, we have $\left\|\rho(t)-e^{t \mathcal{L}_{\mathrm{MM}} \mathcal{P}}[\rho(0)]\right\|=\|\left[e^{t_{n} \mathcal{L}}-\mathcal{P}\right]^{n}(\mathcal{I}-$ $\mathcal{P})[\rho(0)]\|\leqslant\| e^{t_{n} \mathcal{L}}-\mathcal{P}\left\|^{n}\right\| \mathcal{I}-\mathcal{P} \|$. Since we have $\left\|\rho\left(t_{n}\right)-\mathcal{P}[\rho(0)]\right\| \leqslant \mathcal{C}_{\mathrm{MM}}\left(t^{\prime \prime}, t^{\prime}\right) \quad[$ cf. $\quad$ Eq. $\quad(10)], \quad$ while $\|\mathcal{I}-\mathcal{P}\| \leqslant\|\mathcal{I}\|+\|\mathcal{P}\|=2+\mathcal{C}_{+} \quad\left[\begin{array}{ccc}\text { cf. } & \text { Eq. } & (12)], \quad \text { we }\end{array}\right.$ arrive at $\left\|\rho(t)-e^{t \mathcal{L}_{\mathrm{MM}}} \mathcal{P}[\rho(0)]\right\| \leqslant \mathcal{C}_{\mathrm{MM}}^{n}\left(t^{\prime \prime}, t^{\prime}\right)\left(2+\mathcal{C}_{+}\right) \lesssim$ $2 \mathcal{C}_{\mathrm{MM}}^{n}\left(t^{\prime \prime}, t^{\prime}\right)$

[73] For an integer $n$ such that $t^{\prime \prime}+n\left(t^{\prime}-t^{\prime \prime}\right) \leqslant t \leqslant$ $t^{\prime}+n\left(t^{\prime}-t^{\prime \prime}\right), \quad$ we have $\left\|e^{t \mathcal{L}}-\mathcal{P}\right\| \leqslant\left\|(\mathcal{I}-\mathcal{P}) e^{t \mathcal{L}}\right\|+$ $\left\|\mathcal{P} e^{t \mathcal{L}}-\mathcal{P}\right\|, \quad$ where $\quad\left\|(\mathcal{I}-\mathcal{P}) e^{t \mathcal{L}}\right\| \leqslant\left\|(\mathcal{I}-\mathcal{P}) e^{t^{\prime \prime}} \mathcal{L}\right\| \leqslant$ $\left(2+\mathcal{C}_{+}\right) \mathcal{C}_{\mathrm{MM}}$ due to $\left\|e^{\left(t-t^{\prime \prime}\right) \mathcal{L}}\right\|=1$, while $\| \mathcal{P} e^{t \mathcal{L}}-$ $\mathcal{P}\left\|\leqslant \sum_{n^{\prime}=1}^{n}\right\| \mathcal{P} e^{\left[t-\left(n^{\prime}-1\right)\left(t^{\prime}-t^{\prime \prime}\right)\right] \mathcal{L}}-\mathcal{P} e^{\left[t-n^{\prime}\left(t^{\prime}-t^{\prime \prime}\right)\right] \mathcal{L}} \|+$ $\left\|\mathcal{P} e^{t-n\left(t^{\prime}-t^{\prime \prime}\right) \mathcal{L}}-\mathcal{P}\right\| \leqslant n\left\|\mathcal{P}\left[e^{t-n\left(t^{\prime}-t^{\prime \prime}\right) \mathcal{L}}-\mathcal{P}\right]\right\| \leqslant(n+1)$ $\left(1+\mathcal{C}_{+}\right) \mathcal{C}_{\mathrm{MM}}$

[74] P. Zanardi, Dissipative dynamics in a quantum register, Phys. Rev. A 56, 4445 (1997).

[75] P. Zanardi and M. Rasetti, Noiseless Quantum Codes, Phys. Rev. Lett. 79, 3306 (1997).

[76] D. A. Lidar, I. L. Chuang, and K. B. Whaley, DecoherenceFree Subspaces for Quantum Computation, Phys. Rev. Lett. 81, 2594 (1998).

[77] E. Knill, R. Laflamme, and L. Viola, Theory of Quantum Error Correction for General Noise, Phys. Rev. Lett. 84, 2525 (2000).

[78] P. Zanardi, Stabilizing quantum information, Phys. Rev. A 63, 012301 (2000). 
[79] P. Zanardi and L. Campos Venuti, Coherent Quantum Dynamics in Steady-State Manifolds of Strongly Dissipative Systems, Phys. Rev. Lett. 113, 240406 (2014).

[80] P. Zanardi and L. Campos Venuti, Geometry, robustness, and emerging unitarity in dissipation-projected dynamics, Phys. Rev. A 91, 052324 (2015).

[81] P. Zanardi, J. Marshall, and L. Campos Venuti, Dissipative universal Lindbladian simulation, Phys. Rev. A 93, 022312 (2016).

[82] V. Popkov, S. Essink, C. Presilla, and G. Schütz, Effective quantum zeno dynamics in dissipative quantum systems, Phys. Rev. A 98, 052110 (2018).

[83] We have $\left|c_{k}^{(l)}-\sum_{l=1}^{m} p_{l} c_{k}^{(l)}\right|=\mid \operatorname{Tr}\left(L_{k}\{\mathcal{P}[\rho(0)]-\right.$ $\left.\left.\sum_{l=1}^{m} p_{l} \rho_{l}\right\}\right)\left|\leqslant\left\|L_{k}\right\|_{\max }\right| \mid \mathcal{P}[\rho(0)]-\sum_{l=1}^{m} p_{l} \rho_{l} \| \leqslant$

$\|\mathcal{P}[\rho(0)]-\rho(t)\|+\left\|\rho(t)-\sum_{l=1}^{m} p_{l} \rho_{l}\right\|$, where we used the left-eigenmatrix normalization $\left\|L_{k}\right\|_{\max } \leqslant c_{k}^{\max }-c_{k}^{\min }=1$ with $c_{k}^{\max }$ and $c_{k}^{\min }$ denoting the maximal and minimal eigenvalue of $L_{k}$. We then choose time $t$ within the metastable regime for which $\|\mathcal{P}[\rho(0)]-\rho(t)\|$ is minimal.

[84] We have $\operatorname{det} \mathbf{C}=\operatorname{det} \overline{\mathbf{C}}$, where $(\overline{\mathbf{C}})_{l-1, k-1}=c_{k}^{(l)}-c_{k}^{(1)}, k, l=$ $2, . ., m$, encodes the coefficients for the simplex with the vertex of $\tilde{\rho}_{1}$ shifted to the origin.

[85] K. Życzkowski and H.-J. Sommers, Induced measures in the space of mixed quantum states, J. Phys. A 34, 7111 (2001).

[86] K. Życzkowski and H.-J. Sommers, Hilbert-Schmidt volume of the set of mixed quantum states, J. Phys. A 36, 10115 (2003).

[87] We note that often the diamond norm, $\|\mathcal{E}-\mathcal{I}\|_{\diamond} \equiv \| \mathcal{E} \otimes \mathcal{I}-$ $\mathcal{I} \otimes \mathcal{I} \|$, is used instead of induced trace norm, $\|\mathcal{E}-\mathcal{I}\| \leqslant$ $\|\mathcal{E}-\mathcal{I}\|_{\diamond}$, to quantify the disturbance caused by a quantum channel $\mathcal{E}$ with respect to the identity channel $\mathcal{I}$.

[88] M. M. Wolf, Quantum Channels and Operations, Guided Tour (2012).

[89] M. A. Nielsen and I. L. Chuang, Quantum Computation and Quantum Information, 10th Anniversary ed. (Cambridge University Press, Cambridge, UK, 2010).

[90] B. Gaveau, A. Lesne, and L. S. Schulman, Spectral signatures of hierarchical relaxation, Phys. Lett. A 258, 222 (1999).

[91] $\|\mathbf{X}\|_{1}$ is the matrix norm induced by the L1 norm of vectors. For a vector $\mathbf{v}$ and a matrix $\mathbf{X}$, we have $\quad\|\mathbf{X v}\|_{1}=\sum_{k=1}^{m}\left|\sum_{l=1}^{m} X_{k l} v_{l}\right| \leqslant \sum_{k, l=1}^{m}\left|X_{k l} v_{l}\right| \leqslant$ $\left(\max _{1 \leqslant l \leqslant m} \sum_{k=1}^{m}\left|X_{k l}\right|\right) \sum_{l=1}^{m}\left|v_{l}\right| \equiv\|\mathbf{X}\|_{1}|| \mathbf{v} \|_{1}$, and the inequality can be saturated by considering basis vectors.

[92] We note that an analogous approximation of the stationary state can be also obtained by considering non-Hermitian perturbation theory for $\mathbf{p}_{\mathrm{ss}}$ with respect to perturbation $\mathbf{W}-\tilde{\mathbf{W}}$ of $\tilde{\mathbf{W}}$ (see Sec. E2d in Ref. [55]).

[93] Due to the exponential decay of $\left\|\tilde{\mathbf{P}}_{\mathrm{ss}}-e^{n \tau} \tilde{\mathbf{W}}_{\|_{1}} \leqslant\right\| \tilde{\mathbf{P}}_{\mathrm{ss}}-$ $e^{\tilde{\tau} \tilde{\mathbf{W}}} \|_{1}^{n}$ and $\left\|\tilde{\mathbf{P}}_{\mathrm{ss}}-e^{\tau \tilde{\mathbf{W}}}\right\|_{1} \leqslant\left(1+\tilde{\mathcal{C}}_{\mathrm{cl}} / 2\right)\left\|e^{\tau \mathcal{L}}-\mathcal{P}_{\mathrm{ss}}\right\|$, this will be typically implied by Eq. (40); cf. Sec. B3c in Ref. [55].

[94] A. Smirne, D. Egloff, M. G. Díaz, M. B. Plenio, and S. F. Huelga, Coherence and nonclassicality of quantum Markov processes, Quantum Sci. Technol. 4, 01LT01 (2018).

[95] T. Kato, Perturbation Theory for Linear Operators (Springer, Berlin, 1995).

[96] L. S. Levitov, H. Lee, and G. B. Lesovik, Electron counting statistics and coherent states of electric current, J. Math. Phys. 37, 4845 (1996).
[97] Y. V. Nazarov and N. A. T. O. S. A. Division, Quantum Noise in Mesoscopic Physics, NATO Science Series: Mathematics, Physics and Chemistry (Springer, New York, 2003).

[98] M. Esposito, U. Harbola, and S. Mukamel, Nonequilibrium fluctuations, fluctuation theorems, and counting statistics in quantum systems, Rev. Mod. Phys. 81, 1665 (2009).

[99] C. Flindt, C. Fricke, F. Hohls, T. Novotný, K. Netočný, T. Brandes, and R. J. Haug, Universal oscillations in counting statistics, Proc. Natl. Acad. Sci. USA 106, 10116 (2009).

[100] J. M. Hickey, S. Genway, I. Lesanovsky, and J. P. Garrahan, Thermodynamics of quadrature trajectories in open quantum systems, Phys. Rev. A 86, 063824 (2012).

[101] J. M. Hickey, S. Genway, I. Lesanovsky, and J. P. Garrahan, Time-integrated observables as order parameters for full counting statistics transitions in closed quantum systems, Phys. Rev. B 87, 184303 (2013).

[102] V. Lecomte, C. Appert-Rolland, and F. van Wijland, Thermodynamic formalism for systems with Markov dynamics, J. Stat. Phys. 127, 51 (2007).

[103] J. P. Garrahan, R. L. Jack, V. Lecomte, E. Pitard, K. van Duijvendijk, and F. van Wijland, Dynamical First-Order Phase Transition in Kinetically Constrained Models of Glasses, Phys. Rev. Lett. 98, 195702 (2007).

[104] C. Maes, Frenesy: Time-symmetric dynamical activity in nonequilibria, Phys. Rep. 850, 1 (2020).

[105] J. P. Garrahan, Aspects of nonequilibrium in classical and quantum systems: Slow relaxation and glasses, dynamical large deviations, quantum nonergodicity, and open quantum dynamics, Physica A 504, 130 (2018).

[106] R. L. Jack, Ergodicity and large deviations in physical systems with stochastic dynamics, Eur. Phys. J. B 93, 74 (2020)

[107] $H$ can be further replaced by $H-c \mathbb{1}$, with $c$ being a real constant and the norm minimized with respect to $c$ in the corrections; see Sec. E3d in Ref. [55].

[108] S. Gammelmark and K. Mølmer, Fisher Information and the Quantum Cramér-Rao Sensitivity Limit of Continuous Measurements, Phys. Rev. Lett. 112, 170401 (2014).

[109] K. Macieszczak, M. Guţă, I. Lesanovsky, and J. P. Garrahan, Dynamical phase transitions as a resource for quantum enhanced metrology, Phys. Rev. A 93, 022103 (2016).

[110] In Eq. (71) we require $n^{\prime} \mathcal{C}_{\mathrm{cl}} \ll 1$ for all prime factors $n^{\prime}$ of $n$ (see Sec. G2a in Ref. [55]).

[111] J. E. Gough, T. S. Ratiu, and O. G. Smolyanov, Noethers theorem for dissipative quantum dynamical semi-groups, J. Math. Phys. 56, 022108 (2015).

[112] When momenta of plane waves over candidate state cycles do not correspond to arguments of symmetry eigenvalues for the low-lying eigenmodes, $\mathbf{C}_{\mathbf{U}}$ is not invertible and $\operatorname{det} \mathbf{C}_{\mathbf{U}}=0$.

[113] This follows from the spectrum of any operator unchanged under the action of the symmetry, and $\mathcal{U}\left[\tilde{P}_{\pi^{n}(l)}^{\prime}\right]=$ $\tilde{P}_{\pi^{n+1}(l)}^{\prime}$. Alternatively, the minimal eigenvalue can be found from the wave-plane basis by considering averages of $\sum_{n=0}^{d_{l}-1}\left(e^{-i 2 \pi \frac{k}{d}}\right)^{n} \mathcal{U}^{\dagger n}(|\psi\rangle\langle\psi|)$ maximized over $|\psi\rangle$, which can be restricted to $U^{d_{l}}$ symmetric states [cf. Eq. (80b)].

[114] R. Blume-Kohout, H. K. Ng, D. Poulin, and L. Viola, Characterizing the Structure of Preserved Information in Quantum Processes, Phys. Rev. Lett. 100, 030501 (2008). 
[115] R. Blume-Kohout, H. K. Ng, D. Poulin, and L. Viola, Information-preserving structures: A general framework for quantum zero-error information, Phys. Rev. A 82, 062306 (2010).

[116] J. A. Holbrook, D. W. Kribs, and R. Laflamme, Noiseless subsystems and the structure of the commutant in quantum error correction, Quant. Inf. Proc. 2, 381 (2004).

[117] M.-D. Choi and D. W. Kribs, Method to Find Quantum Noiseless Subsystems, Phys. Rev. Lett. 96, 050501 (2006).

[118] W. H. Żurek, Preferred states, predictability, classicality, and the environment-induced decoherence, Prog. Theor. Phys. 89, 281 (1993).

[119] C. I. Bengtsson and K. Życzkowski, Geometry of Quantum States: An Introduction to Quantum Entanglement (Cambridge University Press, Cambridge, UK, 2006).

[120] For $n_{k}$ not divisible by 4; otherwise, see Sec. H5c in Ref. [55].

[121] H. Touchette, The large deviation approach to statistical mechanics, Phys. Rep. 478, 1 (2009).

[122] L. O. Hedges, R. L. Jack, J. P. Garrahan, and D. Chandler, Dynamic order-disorder in atomistic models of structural glass formers, Science 323, 1309 (2009).

[123] C. Giardina, J. Kurchan, V. Lecomte, and J. Tailleur, Simulating rare events in dynamical processes, J. Stat. Phys. 145, 787 (2011).
[124] The timescale $\tau(s)$ of the relaxation toward $\rho_{\mathrm{ss}}(s)$ in the biased dynamics $e^{t \mathcal{L}_{s}}[\rho(0)] / \operatorname{Tr}\left\{e^{t \mathcal{L}_{s}}[\rho(0)]\right\}$ belongs to the metastable regime when Eqs. (63) and (64) hold. Indeed, due to the separation also present in the spectrum of $\mathcal{L}_{s}, \tau(s)$ is approximated by the relaxation time of $\mathbf{W}_{h_{s}}$ of Eq. (62) and negligible corrections from fast modes of $\mathcal{L}$ give $\tau(s) \geqslant t^{\prime \prime}$. Moreover, as $\mathbf{W}$ can be neglected in Eq. (62), $\tau(s) \leqslant t^{\prime}$.

[125] R. S. Burkey and C. D. Cantrell, Discretization in the quasicontinuum, J. Opt. Soc. Am. B 1, 169 (1984).

[126] A. W. Chin, A. Rivas, S. F. Huelga, and M. B. Plenio, Exact mapping between system-reservoir quantum models and semi-infinite discrete chains using orthogonal polynomials, J. Math. Phys. 51, 092109 (2010).

[127] M. P. Woods, R. Groux, A. W. Chin, S. F. Huelga, and M. B. Plenio, Mappings of open quantum systems onto chain representations and Markovian embeddings, J. Math. Phys. 55, 032101 (2014).

[128] J. Iles-Smith, N. Lambert, and A. Nazir, Environmental dynamics, correlations, and the emergence of noncanonical equilibrium states in open quantum systems, Phys. Rev. A 90, 032114 (2014).

[129] M. Gring, M. Kuhnert, T. Langen, T. Kitagawa, B. Rauer, M. Schreitl, I. Mazets, D. A. Smith, E. Demler, and J. Schmiedmayer, Relaxation and prethermalization in an isolated quantum system, Science 337, 1318 (2012). 